\title{
Non-blocking Patricia Tries with Replace Operations
}

\author{
Niloufar Shafiei \\ York University, Department of Computer Science and Engineering
}

\begin{abstract}
This paper presents a non-blocking Patricia trie implementation for an asynchronous shared-memory system using Compare\&Swap. The trie implements a linearizable set and supports three update operations: insert adds an element, delete removes an element and replace replaces one element by another. The replace operation is interesting because it changes two different locations of tree atomically. If all update operations modify different parts of the trie, they run completely concurrently. The implementation also supports a wait-free find operation, which only reads shared memory and never changes the data structure. Empirically, we compare our algorithms to some existing set implementations.
\end{abstract}

\section{INTRODUCTION}

A Patricia trie [22] is a tree that stores a set of keys, which are represented as strings. The trie is structured so that the path from the root to a key is determined by the sequence of characters in the key. So, the length of this path is at most the length of the key (and will often be shorter). Thus, if key strings are short, the height of the trie remains small without requiring any complicated balancing. The simplicity of the data structure makes it a good candidate for concurrent implementations. Patricia tries are widely used in practice. They have applications in routing systems, data mining, machine learning, bioinformatics, etc. [4], [14], [16], [21], [23]. Allowing concurrent access is essential in some applications and can boost efficiency in multicore systems.

We present a new concurrent implementation of $\mathrm{Pa}-$ tricia tries for binary strings using single-word Compare\&Swap (CAS). The operations on the trie are linearizable, meaning they appear to take place atomically [19]. They are also non-blocking (lock-free): some process completes its operation in a finite number of steps even if other processes fail. Wait-free algorithms satisfy the stronger guarantee that every process completes its operation in a finite number of steps.

Our implementation supports wait-free find operations and provides non-blocking insertions and deletions. We also provide a non-blocking replace operation that makes two changes to the trie atomically: it deletes one key and inserts another. If all update operations are occurring at disjoint parts of the trie, they do not interfere with one another.
A Patricia trie can be used to store a set of points in $\mathbb{R}^{d}$. For example, a point in $\mathbb{R}^{2}$ whose coordinates are $(x, y)$ can be represented as key formed by interleaving the bits of $x$ and $y$. (This yields a data structure very similar to a quadtree.) Then, the replace operation can be used to move a point from one location to another atomically. This operation has applications in Geographic Information System [15]. The replace operation would also be useful if the Patricia trie were adapted to implement a priority queue, so that one can change the priority of an element in the queue.

Search trees are another class of data structures that are commonly used to represent sets. When keys are not uniformly distributed, balanced search trees generally outperform unbalanced ones. The reverse is often true when keys are uniformly distributed due to the simplicity of unbalanced search trees. Our empirical results show that the performance of our trie is consistently good in both scenarios. This is because our trie implementation is as simple as an unbalanced search tree but also keeps trees short. For simplicity, we rely on a garbage collector (such as the one provided in Java implementations) that deallocates objects when they are no longer accessible.

For our Patricia trie algorithms, we extend the scheme used in [11] for binary search trees to coordinate processes. Thus, we show that the scheme is more widely applicable. In particular, we extend the scheme so that it can handle update operations that make more than one change to the tree structure. Updates to the same part of the tree help one another to guarantee the non-blocking property. An update first creates a descriptor object that contains enough information about the update, so that other processes can complete the update by reading the descriptor object. As in [11], before an update changes the tree, it flags a small number of nodes to avoid interference with other concurrent updates. (A node is flagged if it has a pointer to a descriptor object, otherwise it is unflagged.) When the update is complete, the flags are removed from nodes that are still in the tree. Searches do not need to check for flags and can therefore traverse the trie very efficiently simply by reading child pointers. Searches in our Patricia trie are wait-free, unlike the searches in [11] because the length of a search path in a Patricia trie is bounded by the length of the key. 
There are several novel features of this work. In our implementation, we design one fairly simple routine that is called to perform the real work of all update operations. In contrast, insert and delete operations in [11] are handled by totally separate routines. This makes our proof of correctness more modular than the proof of [11]. Our techniques and correctness proof can be generalized to other tree-based data structures.

In [11], modifications were only made at the bottom of the search tree. Our new Patricia trie implementation also copes with modifications that can occur anywhere in the trie. This requires proving that changes in the middle of the trie do not cause concurrent search operations passing through the modified nodes to go down the wrong branch. Howley and Jones [20] introduced changes in the middle of a search tree but only to keys stored in internal nodes, not the structure of the tree itself.

In [11], atomic changes had to be done by changing a single pointer. Our replace operation makes two changes to the trie atomically. Both changes become visible at the first CAS operation on a child pointer. This new scheme can be generalized to make several changes to the trie atomically by making all changes visible at a single linearization point. Cederman and Tsigas [9] proposed a non-blocking replace operation for a tree-based data structure, but they require double-CAS (that modifies two non-adjacent locations conditionally).

To summarize:

- We present a non-blocking linearizable Patricia trie.

- We employ one routine to implement the real work of any update operation.

- We present a non-blocking update operation that requires changes to two child pointers using singleword CAS.

- We provide a modular correctness proof that can be adapted for other data structures. We give a sketch of the correctness proof here. A more detailed proof is provided in Appendix.

- We compare our implementation empirically to other existing concurrent data structures.

The remainder of the paper is organized as follows. In Section II, we present related work. We describe the structure of the algorithms and how the operations are implemented in detail in Section III. Section IV provides a sketch of the correctness proof. In Section $V$, we compare our trie to other algorithms empirically. Some concluding remarks are given in Section VI.

\section{RELATED WORK}

Most concurrent data structures are lock-based. However, lock-based implementations have drawbacks such as priority inversion, deadlock and convoying. Two state of the art examples of lock-based implementations of set data structures are the AVL tree by Bronson et al. [6], which maintains an approximately balanced tree, and the self-adjusting binary search tree by Afek et al. [1], which moves frequently accessed nodes closer to the root. Aref and Ilyas [2] described how lock-based implementations could be designed for a class of space-partitioning trees that includes Patricia tries. Lock-coupling can also be applied to implement a concurrent Patricia trie [28].

In this paper, we focus on non-blocking algorithms, which do not use locks. There are two general techniques for obtaining non-blocking data structures: universal constructions (see the related work section of [10] for a recent survey of work on this) and transactional memory [25] (see [17] for a survey). Such general techniques are usually not as efficient as algorithms that are designed for specific data structures.

Tsay and Li [27] gave a general wait-free construction for tree-based data structures. To access a node, a process makes a local copy of the path from the root to the node, performs computations on the local copy, and then atomically replaces the entire path by its local copy. Since this approach copies many nodes and causes high contention at the root, their approach is not very efficient. Barnes [3] presented another general technique to obtain nonblocking implementations of data structures in which processes cooperate to complete operations.

Ellen et al. [11] presented the first non-blocking binary search tree data structure from CAS operations. Their approach has some similarity to the cooperative technique of [3]. As discussed in Section I] our Patricia trie implementation extends the approach used in [11]. Brown and Helga [8] generalized the binary search trees of [11] to non-blocking $k$-ary search trees and compared the non-blocking search trees with the lock-based search tree of Bronson et al. [6] empirically on a multicore system.

Howley and Jones [20] presented a non-blocking search tree from CAS operations using a cooperative technique similar to [11]. Their tree store keys in both leaf and internal nodes. However, search operations sometimes help update operations by performing CASs.

Braginsky and Petrank proposed a non-blocking balanced B+tree from CAS operations [5]. The implementation uses the marking technique of [18] and the flagging technique of [11].

Earlier this year, Prokopec et al. [24] described a nonblocking hash trie that uses CAS operations. Their approach is very different from our implementation. Unlike Patricia tries, in their trie implementation, an internal node might have single child. In their implementation, 


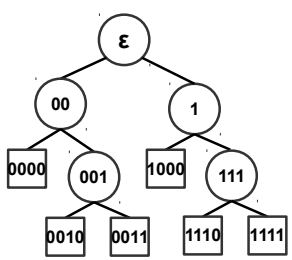

Fig. 1. An example of a Patricia trie. (Leaves are represented by squares and internal nodes are represented by circles.)

nodes have up to $2^{k}$ children (where $k$ is a parameter) and extra intermediate nodes are inserted between the actual nodes of the trie. With $k=5$, the height of their trie is very small, making their implementation very fast when contention is low. However, our experiments suggest that it is not very scalable under high contention. Unlike our implementation, their search operation may perform CAS steps.

Non-blocking implementations of set data structures have also been proposed based on skip lists using CAS operations [12], [13], [26]. A non-blocking skip list (ConcurrentSkipListMap) was then implemented in the Java class library by Doug Lea.

\section{Algorithm DESCRIPTION}

We assume an asynchronous shared-memory system with single-word CAS operations. We first give the sequential specification of the operations. The trie stores a set $D$ of keys from a finite universe $U$. If $v \notin D$, $\operatorname{insert}(v)$ changes $D$ to $D \cup\{v\}$ and returns true; otherwise, it returns false. If $v \in D$, delete $(v)$ changes $D$ to $D-\{v\}$ and returns true; otherwise, it returns false. If $v \in D$ and $v^{\prime} \notin D$, replace $\left(v, v^{\prime}\right)$ changes $D$ to $D-\{v\} \cup\left\{v^{\prime}\right\}$ and returns true; otherwise, it returns false. If $v \in D$, find $(v)$ returns true; otherwise, it returns false. In either case, find $(v)$ does not change $D$. We assume elements of $D$ can be encoded as $\ell$-bit binary strings. (In Section VI we describe how to handle unbounded length keys.)

\section{A. Data Structures}

First, we describe the structure of a binary Patricia trie. (See Figure 11) Each internal node has exactly two children. The elements of $D$ are stored in the leaves of the trie. Each internal node stores a binary string that is the longest common prefix of its children. If a node's label has length $k-1$, then the $k$ th bit of the node's left and right child is 0 and 1 , respectively. The root stores the empty string. The height of the trie is at most $\ell$.

Next, we describe the objects that are used in the implementation (Figure 2). The Patricia trie is represented using Leaf and Internal objects which are subtypes of Node objects. A Node object has a label field representing its binary string, which is never changed after initialization. An Internal object has an array of Node objects of size two, denoted child, that stores pointers to the children of the node.

Each Node object also has an info field that stores a pointer to an Info object that represents an update operation that is in progress at the node. The Info object contains enough information to allow other processes to help the update to complete. The Info object has two subtypes: Flag and Unflag. An Unflag object is used to indicate that no update is in progress at a node. Unflag objects are used instead of null pointers to avoid the ABA problem in the info field of a node. Initially, the info field of each Node object is an Unflag object. We say that a node is flagged or unflagged, depending on whether its info field stores a Flag or Unflag object. The info and child field of an internal node are changed using CAS steps. However, a leaf node gets flagged by writing a Flag object into its info field.

To perform an update operation, first some internal nodes get flagged, then some child fields are changed and then nodes that are still in the trie get unflagged. The nodes that must be flagged to perform an update operation are the internal nodes whose child field will be changed by the update or that will be removed from the trie by the update. Flagging nodes is similar to locking nodes: it avoids having other operations change the part of the trie that would be changed by the update.

A Flag object has a number of fields. The flag field stores nodes to be flagged and the unflag field stores nodes to be unflagged. Before creating a Flag object, an update reads the info field of each node that will be affected by the update before reading that node's child field. This value of the info field is stored in the Flag's oldInfo field, and is used for the CAS that flags the node. This ensures that if the node is successfully flagged, it has not changed since its children were read. Moreover, once it is flagged, its children will not be changed by any other update operation. The boolean flagDone field indicates whether the flagging for the update has been completed. In the case of a replace operation, the rmvLeaf field points to the leaf to be removed by the update after flagging is complete. The actual changes to the trie to be made are described in three more array fields of the Flag object: $p N o d e$, oldChild and newChild. For each $i$, the update should CAS the appropriate child pointer of $p N o d e[i]$ from oldChild $[i]$ to newChild $[i]$. If all nodes are successfully flagged, then the CAS on each child pointer will be guaranteed to succeed because that pointer cannot have changed since the old value was read from it. Thus, like locks, the info field of a node is used to give an 


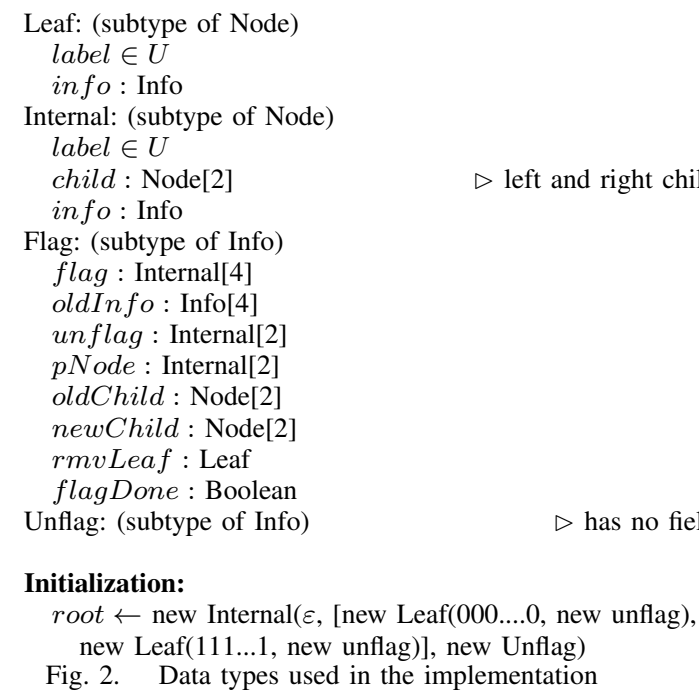

Fig. 2. Data types used in the implementation

operation exclusive permission to change the child field of that node.

For simplicity, the root node of the trie is initially set to an Internal object whose children are two leaf nodes whose labels are the strings $0^{\ell}$ and $1^{\ell}$. We assume the keys $0^{\ell}$ and $1^{\ell}$ cannot be elements of $D$. This ensures that the trie always has at least two leaf nodes and the root node never needs to be replaced. (This avoids some special cases that would occur when the root is a leaf.)

\section{B. Update Operations}

The implementation has three update operations: insert, delete and replace. All three have the same overall structure. The pseudo-code for our implementation is given on page 5. An update $o p$ uses the search routine to find the location(s) in the trie to be changed. It then creates a new Flag object $I$ containing all the information required to complete the update by calling newFlag. If newFlag sees that some node that must be flagged is already flagged with a different Flag $I^{\prime}$, it calls help $\left(I^{\prime}\right)$ at line 110 to try completing the update described by $I^{\prime}$, and then $o p$ retries its update from scratch. Otherwise, $o p$ calls help $(I)$ to try to complete its own update.

As mentioned earlier, flagging nodes ensures exclusive access for changing child pointers. Thus, an update flags the nodes whose child pointers it wishes to change and permanently flags any node that is removed from the trie to avoid applying updates to a deleted portion of the trie

Unlike locks, the Info objects store enough information, so that if an operation dies while nodes are flagged for it, other processes can complete the operation and remove the flags. This ensures that a failed operation cannot prevent others from progressing. To avoid deadlock, if an update must flag more than one internal node, we order the internal nodes by their labels.
The help $(I)$ routine carries out the real work of an update using the information stored in the Flag object $I$. It first uses flag $C A S$ steps to flag some nodes (line 90) by setting their info fields to $I$. If all nodes are flagged successfully, help $(I)$ uses child $C A S$ steps to change the child fields of some internal nodes to perform the update (line 98). Then, it uses unflag CAS steps to unflag nodes that were flagged earlier, except the ones that have been removed from the trie (line 101p by setting their info fields to a new Unflag object. In this case, any nodes deleted by the update remain flagged forever. If any node is not flagged successfully, the attempt to perform the update has failed and backtrack CAS steps are used to unflag any nodes that were flagged earlier (line 105).

If any child CAS step is executed inside help $(I)$, the update is successful and it is linearized at the first such child CAS. If a replace operation performs two different child CAS steps, it first executes a child CAS to insert the new key, and then a child CAS to delete the old key. In this case, the replace also flags the leaf node of the old key before the first child CAS step. We say the leaf is logically removed from the trie at the first child CAS step and any operation that reaches the leaf node after this determines that the key is already removed. We say a node is reachable at time $T$ if there is path from the root to the node at $T$. We say a leaf node is logically in the trie at time $T$ if the node is reachable and not logically removed at $T$. We shall prove that the following invariant holds: The leaf nodes that are logically in the trie at time $T$ contain exactly those keys in the set $D$, according to the sequence of updates that are linearized before $T$.

Whenever a child pointer is changed, the old child is permanently flagged and it is removed from the trie to avoid the ABA problem. (In some cases, this requires the update to add a new copy of the old child to the trie.) When a call to help $(I)$ performs a child CAS on I.pNode $[i]$ (for some $i$ ), it uses I.oldChild $[i]$ as the old value. Since there is no ABA problem, only the first such CAS on I.pNode $[i]$ can succeed. Moreover, we prove that the flagging mechanism ensures that this first CAS does succeed. Since processes might call help $(I)$ to help each other to complete their operations, there might be a group of child CASs on each node. However, the child pointer is changed exactly once for the operation.

\section{Detailed Description of Algorithms}

A $\operatorname{search}(v)$ is used by updates and find to locate key $v$ within the trie. The $\operatorname{search}(v)$ starts from the root node and traverses down the trie. At each step of the traversal, $\operatorname{search}(v)$ chooses the child according to the appropriate bit of $v$ (line 82). The $\operatorname{search}(v)$ stops if it reaches an internal node whose label is not a prefix of $v$. We show 
20) $\operatorname{insert}(v \in U)$

21) while(true)

22) $\quad I \leftarrow$ null

23) $\langle-, p$, node, $-, p I n f o, r m v d\rangle \leftarrow \operatorname{search}(v)$

24) if keyInTrie (node, $v, r m v d$ ) then return false

25) nodeInfo $=$ node.info

26) nodeCopy $\leftarrow$ new copy of node

27)

28)

29)

30)

33)

34)

35)

node Copy $\leftarrow$ new copy of node nodeInfo)

if new Node $\neq$ null then if node is Internal then $[p],[$ node $],[$ new Node], null)

else $I \leftarrow \operatorname{newFlag}([p],[p I n f o],[p],[p]$, [node], [newNode], null)

if $I \neq$ null and help $(I)$ then return true

\section{$\operatorname{delete}(v \in U)$}

while(true)

$I \leftarrow$ null if $g p \neq$ null then [nodeSibling], null)

$$
\text { if } I \neq \text { null and } \operatorname{help}(I) \text { then return true }
$$

replace $\left(v_{d} \in U, v_{i} \in U\right)$

while(true)

$I \leftarrow$ null

nodeInfo $o_{i}=$ node $_{i}$. info

and $p_{i} \neq p_{d}$ then

copy $_{i} \rightarrow$ new copy of node $_{i}$ $v_{i}$, nodeInfo $\mathrm{f}_{i}$ ) node $_{d}$ ) $\left[\right.$ new Node $_{i}$, nodeSibling $\left._{d}\right]$, node $\left._{d}\right)$

else if node $_{i}=$ node $_{d}$ then [new Leaf containing $\left.v_{i}\right]$, null)

else if node $_{i}=p_{d}$ and $p_{i}=g p_{d}$ ) or

$\left(g p_{d} \neq\right.$ null and $\left.p_{i}=p_{d}\right)$ then containing $v_{i}$, nodeSibling . info) $^{\text {in }}$

if new Node $_{i} \neq$ null then $\left[g p_{d}\right],\left[p_{d}\right],\left[\right.$ new Node $\left.e_{i}\right]$, null)

else if node $_{i}=g p_{d}$ then

if newChild $_{i} \neq$ null then containing $v_{i},-$ ) [node $\left.{ }_{i}\right],\left[\right.$ newNode $\left._{i}\right]$, null)

if $I \neq$ null and help $(I)$ then return true
newNode $\leftarrow$ createNode $(\operatorname{cop} y$, new Leaf containing $v$,

$I \leftarrow \operatorname{newFlag}([p$, node $],[p \operatorname{Info}, \operatorname{nodeInfo}],[p]$,

$\langle g p, p$, node, gpInfo, $\operatorname{In} f o, r m v d\rangle \leftarrow \operatorname{search}(v)$

if $\neg$ keyInTrie (node, $v, r m v d$ ) then return false

nodeSibling $\leftarrow$ p.child $[1-(\mid$ p.label $\mid+1)$ th bit of $v]$

$I \leftarrow \operatorname{newFlag}([g p, p],[g p \operatorname{Inf} o, p \operatorname{Info}],[g p],[g p],[p]$,

$\left\langle g p_{d}, p_{d}\right.$, node $_{d}$, gpInfo $\left.o_{d}, p I n f o_{d}, r m v d_{d}\right\rangle \leftarrow \operatorname{search}\left(v_{d}\right)$

if $\neg$ keyInTrie $\left(\right.$ node $\left._{d}, v_{d}, r m v d_{d}\right)$ then return false

$\left\langle-, p_{i}\right.$, node $_{i},-$, pInfo $\left.o_{i},-, \operatorname{rmvd}_{i}\right\rangle \leftarrow \operatorname{search}\left(v_{i}\right)$

if keyInTrie $\left(\right.$ node $\left._{i}, v_{i}, r m v d_{i}\right)$ then return false

nodeSibling $_{d} \leftarrow p_{d}$.child $\left[1-\left(\mid p_{d}\right.\right.$. label $\left.\mid+1\right)$ th bit of $\left.v_{d}\right]$

if $g p_{d} \neq$ null and node $_{i} \notin\left\{\right.$ node $\left._{d}, p_{d}, g p_{d}\right\}$

new Node $_{i} \leftarrow \operatorname{createNode}\left(\operatorname{copy}_{i}\right.$, new Leaf containing

if new Node $_{i} \neq$ null and node $_{i}$ is Internal then

$I \leftarrow \operatorname{newFlag}\left(\left[g p_{d}, p_{d}, p_{i}, \operatorname{node}_{i}\right],\left[\operatorname{gpInfo} o_{d}\right.\right.$, $p I n f o_{d}, p I n f o_{i}$, nodeInfo $\left.o_{i}\right],\left[g p_{d}, p_{i}\right],\left[p_{i}\right.$,

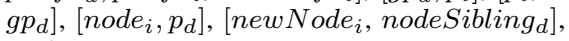

else if new Node $i \neq$ null and node $_{i}$ is Leaf then

$I \leftarrow \operatorname{newFlag}\left(\left[g p_{d}, p_{d}, p_{i}\right],\left[g p I n f o_{d}, p I n f o_{d}\right.\right.$, pInfo $\left.o_{i}\right],\left[g p_{d}, p_{i}\right],\left[p_{i}, g p_{d}\right],\left[\right.$ node $\left._{i}, p_{d}\right]$,

$I \leftarrow \operatorname{newFlag}\left(\left[p_{d}\right],\left[p I n f o_{d}\right],\left[p_{d}\right],\left[p_{d}\right],\left[\right.\right.$ node $\left.e_{i}\right]$,

new Node $_{i} \leftarrow \operatorname{createNode}\left(\right.$ nodeSibling $_{d}$, new Leaf

$I \leftarrow \operatorname{newFlag}\left(\left[g p_{d}, p_{d}\right],\left[g p I n f o_{d}, p I n f o_{d}\right],\left[g p_{d}\right]\right.$,

pSibling $g_{d} \leftarrow g p_{d}$.child $\left[1-\left(\mid g p_{d}\right.\right.$. label $\left.\mid+1\right)$ th bit of $\left.v_{d}\right] 1$ newChild $_{i} \leftarrow$ createNode $\left(\right.$ nodeSibling $_{d}$, pSibling $\left._{d},-\right)$ 123)

new Node $_{i} \leftarrow \operatorname{createNode}\left(\right.$ newChild $_{i}$, new Leaf

if new Node $_{i} \neq$ null then $I \leftarrow \operatorname{newFlag}\left(\left[p_{i}, g p_{d}\right.\right.$, $\left.p_{d}\right],\left[p \operatorname{Inf} o_{i}, g p I n f o_{d}, p I n f o_{d}\right],\left[p_{i}\right],\left[p_{i}\right]$,

Fig. 3. Update operations find $(v \in U)$

73) $\langle-,-$, node, -, -, rmvd $\rangle \leftarrow \operatorname{search}(v)$

74) if keyInTrie (node, $v, r m v d$ ) then return true

75) else return false

76) $\operatorname{search}(v \in U)$

77) $\langle p, p \operatorname{Info}\rangle \leftarrow\langle$ null, null $\rangle$

78) node $\leftarrow$ root

79) while (node is Internal and node.label is prefix of $v$ )

80) $\langle g p, g p I n f o\rangle \leftarrow\langle p, p I n f o\rangle$

$\langle p, p I n f o\rangle \leftarrow\langle$ node, node.info $\rangle$

node $\leftarrow$ p.child $[(\mid p$.label $\mid+1)$ th bit of $v]$

if node is Leaf then $\quad \triangleright$ if Leaf is replaced

$r m v d \leftarrow \operatorname{logicallyRemoved}($ node.info)

return $\langle g p, p$, node, gpInfo, $p$ Info, rmvd $\rangle$

86) $\operatorname{help}(I$ : Flag $)$

87) $i \leftarrow 0$

88) doChildCAS $\leftarrow$ true

89) while $(i<\mid$ I.flag $\mid$ and doChildCAS)

90) CAS(I.flag $[i]$.info, I.oldInfo $[i], I)$

doChildCAS $\leftarrow($ I.flag $[i]$.info $=I)$

$\triangleright$ flag CAS

$i \leftarrow i+1$

if $d o C h i l d C A S$ then

I.flagDone $\leftarrow$ true

if I.rmvLeaf $\neq$ null then I.rmvLeaf.info $\leftarrow I$

for $i=0$ to $(\mid$ I.pNode $\mid-1)$

$k \leftarrow(\mid$ I.pNode $[i]$.label $\mid+1)$ th bit of

I.newChild $[i]$.label

CAS(I.pNode $[i]$.child $[k]$, I.oldChild $[i]$, I.newChild $[i])$

if I.flagDone then

for $i=(\mid$ I.unflag $\mid-1)$ down to 0

CAS(I.unflag $[i]$.info, I, new Unflag) $\triangleright$ unflag CAS return true

else

for $i=(\mid I$. flag $\mid-1)$ down to 0

CAS(I.flag $[i]$.info, $I$, new Unflag) $\triangleright$ backtrack CAS return false

107) newFlag(flag, oldInfo, unflag, pNode, oldChild, newChild, rmvLeaf)

108) for $i=0$ to $(\mid$ oldInfo $\mid-1)$,

109) if oldInfo[ $i]$ is Flag then

110) help(oldIn fo $[i]$ )

111) return null

112) if flag has duplicates with different values in oldInfo then

113) return null

114) else remove duplicates in flag and unflag (and corresponding entries of oldInfo)

115) sort elements of flag and permute elements of oldInfo

116) return new Info(flag, oldInfo, unflag, $p$ Node, oldChild, newChild, rmvLeaf, false)

117) createNode node $_{1}$ : Node, node $_{2}$ : Node, in fo: Info)

118) if node .label is prefix of node $_{2}$.label or node $_{2}$.label is prefix of node 1 .label then

119) if info is Flag then $\operatorname{help}($ info)

120) return null

121) else return new Internal whose children are node $_{1}$ and node $_{2}$

22) logicallyRemoved $(I:$ Info)

if $I$ is Unflag then return false

124) return (I.oldChild[0] not in I.pNode[0].child)

125) keyInTrie(node: Node, $v \in U, r m v d$ : Boolean)

126) return (node is Leaf and node.label $=v$ and $r m v d=$ false)

Fig. 4. The find operation and additional subroutines 
that any node visited by the search was reachable at some time during the search. If the $\operatorname{search}(v)$ does not return a leaf containing $v$, there was a time during the search when no leaf containing $v$ was reachable. Moreover, the node that is returned is the location where an insert would have to put $v$. If $\operatorname{search}(v)$ reaches a leaf node and the leaf node is logically removed by a replace operation, $\operatorname{search}(v)$ sets rmvd to true (line 84,.

As we shall see, update operations must change the child pointers of the parent or grandparent of the node returned by search. The search operation returns $g p, p$ and node, the last three nodes reached (where $p$ stands for parent and $g p$ stands for grandparent). A search also returns the values $g p I n f o$ and $p I n f o$ that it read from the info fields of $g p$ and $p$ before reading their child pointers. More formally, if $\operatorname{search}(v)$ returns $\langle g p, p$, node, gpInfo, $p$ Info, $r m v d\rangle$, it satisfies the following post-conditions. (1) At some time during $\operatorname{search}(v)$, gp.info was gpInfo (if $g p$ is not null). (2) Then, at some later time during $\operatorname{search}(v), p$ was a child of $g p$ (if $g p$ is not null). (3) Then, at some later time during $\operatorname{search}(v)$, p.info was $p I n f o$. (4) Then, at some later time during $\operatorname{search}(v)$, p.child $[i]$ was node for some $i$. (5) $($ p.label $) \cdot i$ is a prefix of $v$. (6) If node is an internal node, node.label is not a prefix of $v$. (7) If rmvd is true, node is logically removed at some time during $\operatorname{search}(v)$. (8) If rmvd is false, node is logically in the trie at some time during $\operatorname{search}(v)$.

After calling search, an update uses the newFlag routine to create a Flag object. For each node that the update must flag, a value read from the info field during search of the node is passed to newFlag as the old value to be used in the flag CAS step. The old value for a flag CAS was read before the old value for the corresponding child CAS, so if the flag CAS succeeds, then the node's child field has not been changed since the last time its old value was read. The newFlag routine checks if all old values for info fields are Unflag objects (line 109). If some info field is not an Unflag object, then there is some other incomplete update operating on that node. The newFlag routine tries to complete the incomplete update (line 110), and then returns null, which causes the update to restart. In some cases of the replace operation that change the trie in two steps, $g p$ returned by the first search might be equal to $p$ returned by the second search. So, the operation might send duplicate elements to get flagged to the newFlag routine. If the duplicate elements do not have the same old values, their child fields might have changed since the operation read them, so newFlag returns null and the operation starts over (line 112 113 . Otherwise, only one copy of each duplicate element is

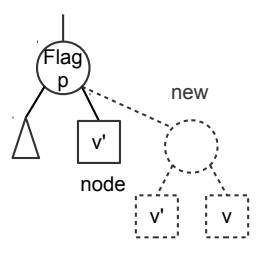

insert(v) - Case 1

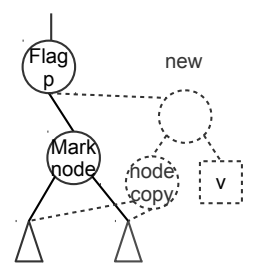

insert(v) - Case 2

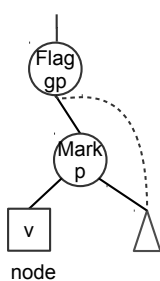

delete(v)
Fig. 5. Different cases of $\operatorname{insert}(v)$ and delete $(v)$. (Triangles are either a leaf node or a subtree. The dotted lines are the new child pointers that replace the old child pointers (solid lines) and the dotted circles are newly created nodes.)

kept (line 114). The newFlag routine sorts the nodes to be flagged (to avoid deadlocks) and returns the new Flag object (line 115 116.

After an update $u$ creates a Flag object $I$, it calls help $(I)$. This routine attempts to complete the update. First, it uses CAS steps to put the Flag object $I$ in the info field of the nodes to be flagged (line 90.). If all nodes are flagged successfully, the flagDone field of the Flag object is set to true (line 94). The value of the flagDone field is used to coordinate processes that help the update. Suppose a process $p$ is executing help $(I)$. After $p$ performs a flag CAS on a node $x$, if it sees a value different from $I$ in the $x$ 's info field, there are two possible cases. The first case is when all nodes were already successfully flagged for $I$ by other processes running $\operatorname{help}(I)$, and then $x$ was unflagged before $p$ tries to flag $x$. (Prior to this unflagging, some process performed the child CAS steps of $I$ successfully.) The second case is when no process flags $x$ successfully for $I$. Since the flagDone field of $I$ is only set to true after all nodes are flagged successfully, $p$ checks the value of the flagDone field to determine which case happened. If flagDone is true, the modifications to the trie for update $u$ have been made. If flagDone is false, the update operation cannot be successfully completed, so all internal nodes that got flagged earlier are unflagged by the back-tracking CAS steps at line 104 106 and the update $u$ will have to start over.

After flagging all nodes successfully and setting I.flagDone, if I.rmvLeaf is a leaf, its info field is set to $I$ (line 95). Only the two-step replace operations flag a leaf. Then, help $(I)$ changes the child fields of nodes in I.pNode using child CASs (line 96 98). Finally, help $(I)$ uses unflag CASs to unflag the nodes in I.unflag and returns true (line 99 102).

The $\operatorname{insert}(v)$ routine first calls $\operatorname{search}(v)$. Let $\langle-, p$, node,,,$-- r m v d\rangle$ be the result returned by $\operatorname{search}(v)$. If node is a leaf containing $v$ and $r m v d$ is false, insert $(v)$ returns false since the trie already contains $v$ (line 24). Otherwise, the insertion attempts to replace node with a node created line 121 , whose children are a new leaf 
node containing $v$ and a new copy of node. (See Figure 5.) Thus, the parent $p$ of node must be flagged. A new copy of node is used to avoid the ABA problem. If node is an internal node, since node is replaced by a new copy, insert $(v)$ must flag node permanently (line 30).

The delete $(v)$ routine first calls $\operatorname{search}(v)$. Let $\langle g p, p$, node, -, -, rmvd $\rangle$ be the result returned by the $\operatorname{search}(v)$. If node is not a leaf node containing $v$ or rmvd is true, delete $(v)$ returns false since the trie does not contain $v$ (line 37). Then, delete $(v)$ replaces $p$ by the sibling of node. (See Figure 5) So, delete $(v)$ must flag the grandparent $g p$ of node and mark $p$ (line 40).

A replace works as follows. First, replace $\left(v_{d}, v_{i}\right)$ calls $\operatorname{search}\left(v_{d}\right)$ and $\operatorname{search}\left(v_{i}\right)$, which return $\left\langle g p_{d}, p_{d}\right.$, node $e_{d}$, ,,$\left.-- r m v d_{d}\right\rangle$ and $\left\langle-, p_{i}\right.$, node $\left._{i},-,-, r m v d_{i}\right\rangle$. The replace checks that $v_{d}$ is in the trie and $v_{i}$ is not, as in the insert and delete operations (line 45 48 ). If either test fails, the replace returns false.

If $\operatorname{insert}\left(v_{i}\right)$ and delete $\left(v_{d}\right)$, as described in Figure 5. would not overlap, replace $\left(v_{d}, v_{i}\right)$ is done by two child CAS steps and is linearized at the first of these two changes. This is called the general case of replace. Situations when the insertion and deletion would occur in overlapping portions of the trie are handled as special cases as shown in Figure 6. In the special cases, the replace changes the trie with one child CAS.

In the general case of the replace operation (line F 57), we create a Flag object which instructs the help routine to perform the following actions. The replace flags the same nodes that an $\operatorname{insert}\left(v_{i}\right)$ and a $\operatorname{delete}\left(v_{d}\right)$ would flag. After flagging these nodes, the leaf node $e_{d}$ also gets flagged. Then, $v_{i}$ is added to the trie, as $\operatorname{in} \operatorname{insert}\left(v_{i}\right)$. When the new leaf node is added, the leaf node $_{d}$, which contains $v_{d}$, becomes logically removed, but not physically removed yet. Then, node $_{d}$ is physically deleted

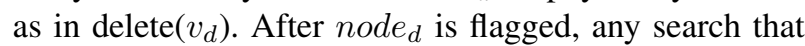
reaches node ${ }_{d}$ checks if $p_{i}$ is a parent of the old child

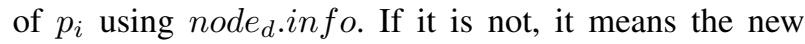
leaf containing $v_{i}$ is already inserted and the operation behaves as if $v_{d}$ is already removed.

There are four special cases of replace $\left(v_{d}, v_{i}\right)$ where the changes required by the insertion and deletion are on the overlapping portions of the trie and the replace operation is done using one child CAS step. Although the code for these cases looks somewhat complicated, it simply implement the actions described in Figure 6 by creating a Flag object and calling help. The insertion of $v_{i}$ replaces $n_{0} e_{i}$ by a new node. The cases when the deletion must remove node $_{i}$ or change node nechild $_{i}$ are handled as special cases. So, the case that node $_{d}=$ node $_{i}$ is one special case (line 58,59). In the deletion,

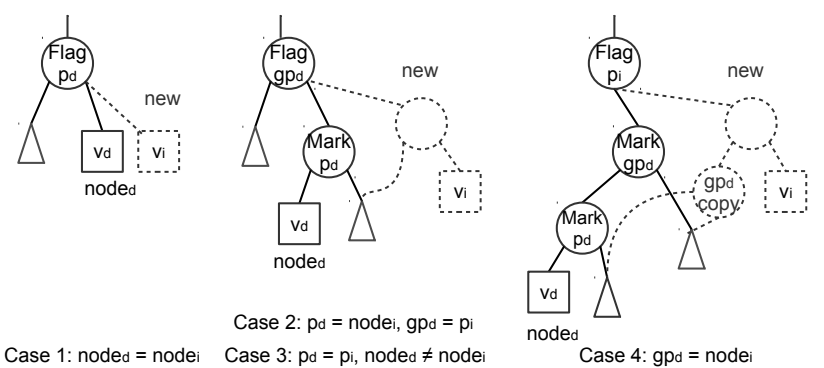

Fig. 6. Special cases of replace $\left(v_{d}, v_{i}\right)$.

$p_{d}$ is removed, so the case that $p_{d}=$ node $_{i}$ or $p_{d}=p_{i}$ are also handled as a special case (line 60 64). In the deletion, gp.child is changed. So, the last special case is when $g p_{d}=$ node $_{i}$ (line 65 70). In all special cases, node $_{i}$ is replaced by a new node. Here, we explain one special case in detail. The others are handled in a similar way. In case 2, $p_{d}=$ node $_{i}$ and $g p_{d}=p_{i}$ (line 60). Since node $_{i}$ must be replaced, a new copy of node $_{i}$ is made. So, replace $\left(v_{d}, v_{i}\right)$ flags $g p_{d}$ and marks node and replaces node ${ }_{i}$ with a new internal node whose nonempty children are a new leaf node containing $v_{i}$ and the sibling of node $_{d}$ (line 60 64).

\section{Algorithm Correctness}

A detailed proof of correctness is provided in Appendix. It is quite lengthy, so we can only provide a brief sketch here. First, we define the linearization point of each operation. Let $\langle-,-$, node, -, -, rmvd $\rangle$ be the result returned by a search. If node is a leaf containing $v$ and $r m v d$ is false, we prove there is a time during the search when node is logically in the trie and the search is linearized at that time. Otherwise, we show there is a time during the search when no leaf containing $v$ is logically in the trie and the search is linearized at that time. If an update returns false, it is linearized at the linearization point of the search that caused the update to fail. Let $I$ be a Flag object created by an update. If a child CAS performed by any call to help $(I)$ is executed, the update is linearized at the first such child CAS. Next, we sketch the correctness proof in four parts.

Part 1 is the heart of the proof. The goal of Part 1 is to prove that, for any Flag object $I$, the successful CAS steps performed by all calls to help $(I)$ proceed in the expected order. (See Figure 7). First, the flag CAS steps are performed on nodes in order, according to the nodes' labels. We prove that only the first flag CAS (by any of the helpers) on each node can succeed. If one if these fails, then the nodes that have been flagged are unflagged by backtrack CAS steps and all calls to $\operatorname{help}(I)$ return false, indicating that the attempt at performing the update has failed. Otherwise, the child CAS steps are performed, and then the unflag CAS steps remove flags from nodes 
that are still reachable. If several helpers perform one of these CAS steps, we prove that the first helper succeeds and no others do. In this case, all calls to help $(I)$ return true.

In Part 1, we first prove that the post-conditions of the search described in Section III-C are satisfied. Then, we prove that each type of update preserves the main invariant of the Patricia trie data structure: if $x$.child $[i]=$ $y$, then $(x . l a b e l) \cdot i$ is a prefix of y.label. Thus, the structure is a correct trie. (This also implies that labels of reachable nodes are distinct, so flagging can be done in order by labels of nodes to avoid deadlock.) We show that the ABA problem on the info fields is avoided because whenever an info field is changed, it is set to a newly created Flag or Unflag object. Then, we show that the CAS steps succeed in the correct order even if they are performed by helpers.

We say that a node is marked if its info field is a Flag object $I$ and the node does not appear in I.unflag and some call to help $(I)$ has performed a child CAS. We show that if an internal node is removed from the trie, it is marked at all times after that. After a node is removed, it is never inserted into the trie again. Next, we show that the ABA problem on the child fields is avoided because whenever a child pointer is changed, the old child is permanently removed from the trie. The proofs of the lemmas in Part 1 are mostly focused on the structure of the help routine. So, any new update that preserves the main invariants of the trie can be added with minor changes to the correctness proof.

Part 2 proves that search operations are linearized correctly. First, we show that each node a search visits was reachable at some time during the operation. Let $\langle$-, -, node, -, -, rmvd $\rangle$ be the result returned by a $\operatorname{search}(v)$. If node is a leaf containing $v$ and $r m v d$ is false, we show that node was reachable and not logically removed at some time during the search. The search is linearized at that time. If node is not a leaf containing $v$ or node is a leaf containing $v$ but rmvd is true, we show that there is a time during the search that no leaf containing $v$ is logically in the trie. The search is linearized at that time.

Part 3 proves that update operations are linearized correctly. Let $T$ be the linearization point of a successful update operation. Since all nodes are flagged successfully, no other concurrent update can change a flagged node's child between the time when the info field of node is read for the last time during the search and the time the node is unflagged. Thus, only the child CAS steps of the update would change that part of the trie during that period of the time. Flagging ensures that the first child CAS of the update has the effect of implementing precisely the change shown in Figure 5 or 6 atomically.

Part 4 proves that the implementation is non-blocking. To derive a contradiction, assume after time $T$, no operation terminates or fails. Let $I$ be a Flag object created by an update that is running after $T$. If a call to $\operatorname{help}(I)$ returns true, the update terminates, so after $T$, all calls to $\operatorname{help}(I)$ return false. Thus, all calls to help $(I)$ set doChildCAS to false because they failed to flag an internal node successfully after $T$. Consider the group of all calls to help $(I)$. We say the group blames an internal node which is the first node that no call to help $(I)$ could flag successfully. Let $g_{0}, \ldots, g_{m}$ be all these groups ordered by the labels of the nodes that they blame. Since $g_{m}$ blames an internal node $x, x$ is flagged by some other group $g_{i}$ where $0 \leq i<m$. Thus, $g_{i}$ blames some other node $y$ whose label is less than $x$. So, $g_{i}$ flags $x$ before attempting to flag $y$, contradicting the fact that $g_{i}$ flags internal nodes in order.

\section{EMPIRICAL EVALUATION}

We experimentally compared the performance of our implementation (PAT) with non-blocking binary search trees (BST) [11], non-blocking k-ary search trees (4ST) [8], ConcurrentSkipListMap (SL) of the Java library, lock-based AVL trees (AVL) [6] and non-blocking hash tries (Ctrie) [24]. For the k-ary search trees, we use the value $k=4$, which was found to be optimal in [8]. Nodes in Ctrie have up to 32 children.

The experiments were executed on a Sun SPARC Enterprise T5240 with 32GB RAM. The machine had two UltraSPARC $\mathrm{T} 2+$ processors, each having eight $1.2 \mathrm{GHz}$ cores, for a total of 128 hardware threads. The experiments were run in Java. The sun JVM version 1.7.0_3 was run in server mode. The heap size was set to $2 \mathrm{G}$. This ensures the garbage collector would not be invoked too often, so that the measurements reflect the running time of the algorithms themselves. Using a smaller heap size affects the performance of BST, 4-ST and PAT more than AVL and SL since they create more objects.

We evaluated the algorithms in different scenarios. We ran most experiments using uniformly distributed random keys. We ran the algorithms using uniformly distributed keys in two different ranges: $\left(0,10^{2}\right)$ to measure performance under high contention and $\left(0,10^{6}\right)$ for low contention. (We also ran the experiments for the key range of $\left(0,10^{3}\right)$ for medium contention, but since the results were very similar to the low contention case, we do not present them here.) We ran experiments with two different operation ratios: $5 \%$ inserts, $5 \%$ deletes and 90\% finds (i5-d5-f90), and 50\% inserts, 50\% deletes and $0 \%$ finds (i50-d50-f0). (We also ran the experiments with 


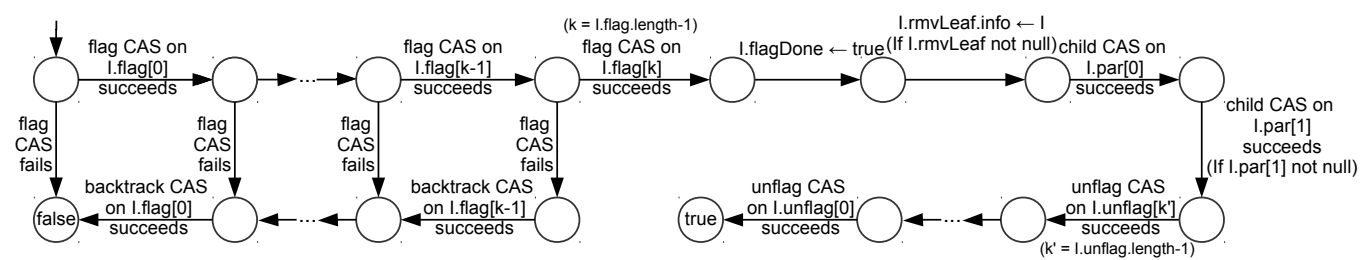

Fig. 7. The correct order of steps inside help $(I)$ for each Flag object $I$. (Steps can be performed by different calls to help $(I)$.)

ratio of $15 \%$ inserts, $15 \%$ deletes and $70 \%$ finds. Since the results were similar to the experiments with the ratio of (i5-d5-f90), we do not present them here.)

Since the replace operation is not used in these sets of experiments, we made some minor optimization to the pseudo-code. For example, we eliminated the rmvd variable in search operations.

Since the Java compiler optimizes its running code, before each experiment, we perform (i50-d50-f0) for ten seconds for each implementation. We start each experiment with a tree initialized to be half-full, created by running updates in the ration i50-d50-f0. Each data point in our graphs is the average of eight 4-second trials. (The error bars in the charts shows the standard deviation.)

For uniformly distributed keys, algorithms scale well under low contention (key range of $\left(0,10^{6}\right)$ ). (See Figure 8.) Under very high contention (key range of $\left(0,10^{2}\right)$ ), most scale reasonably well when the fraction of updates is low, but experience problems when all operation are updates. (See Figure 9) When the range is $\left(0,10^{6}\right)$, Ctrie outperforms all others since the height of the Ctrie is small compared to the others because node can have up to 32 children. However when the range is $\left(0,10^{2}\right)$ and the contention is very high, Ctrie does not scale. Excluding Ctrie, when the range is $\left(0,10^{6}\right)$, PAT, 4-ST and BST outperform AVL and SL. Since updates are more expensive than finds, the throughput is greater for i5-d5-f90 than for i50-d50-f0.

To evaluate the replace operations, we ran an experiment with $10 \%$ inserts, $10 \%$ deletes and $80 \%$ replace operations (i10-d10-r80) and a key range of $\left(0,10^{6}\right)$ on uniformly random keys. (See Figure 10 ) We could not compare these results with other data structure since none provide atomic replace operations. As the chart shows, the replace operation scales well as the number of threads increases.

We also performed some experiments on nonuniformly distributed random keys. To generate nonuniform keys, processes performed operations on sequence of 50 consecutive keys, starting from a randomly chosen key. When keys are not uniformly distributed and key range is $\left(0,10^{6}\right)$, Ctrie and then PAT outperform others greatly since they maintain a fixed height without

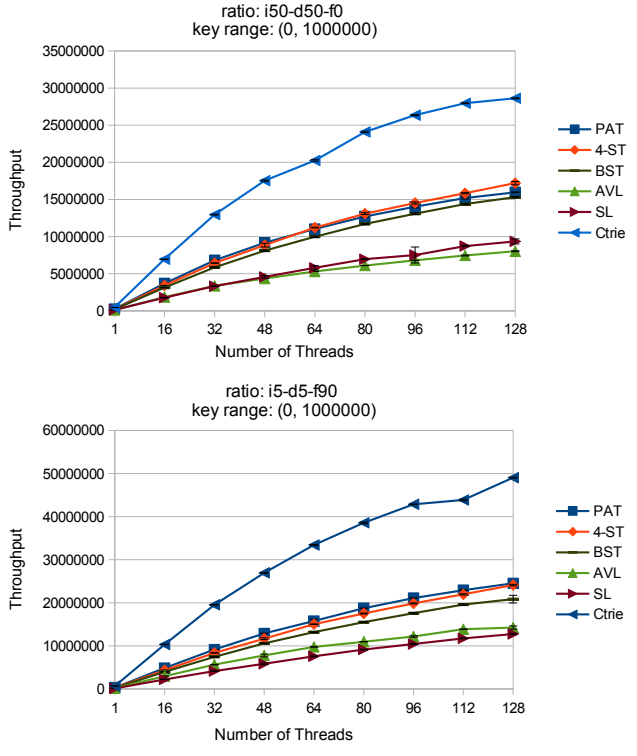

Fig. 8. Uniformly distributed keys
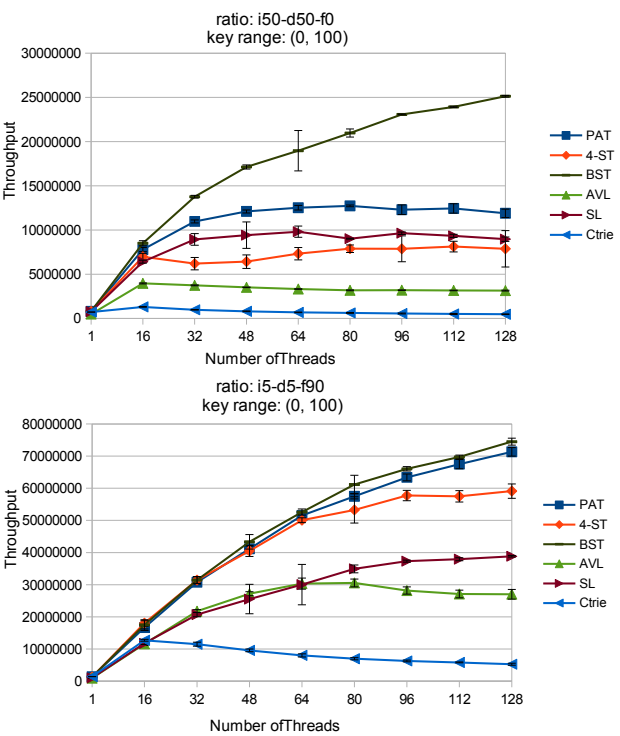

Fig. 9. Uniformly distributed keys

doing expensive balancing operations. (See Figure 11.) Since the results of these experiments for other operations ratios were similar, only the chart for the ratio $\mathrm{i} 15-\mathrm{d} 15-\mathrm{f} 70$ is presented here. Longer sequences of keys degrade the performance of BST and 4-ST even further. 


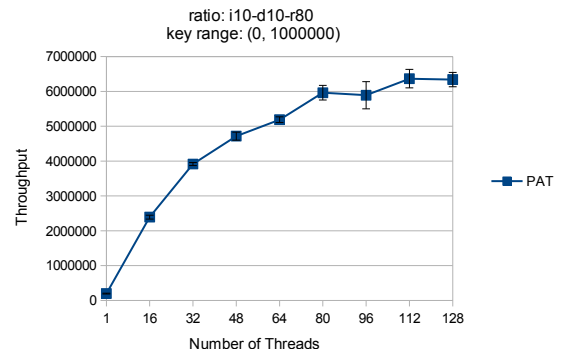

Fig. 10. Replace operations of PAT

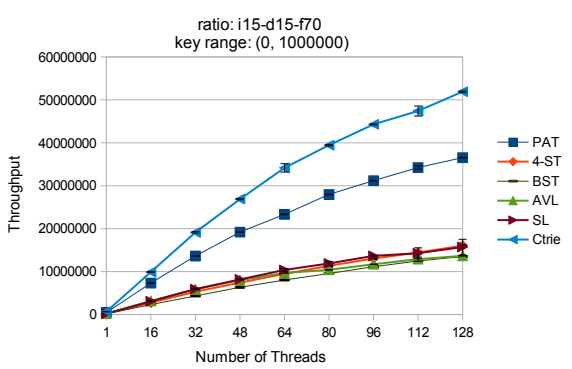

Fig. 11. Non-uniformly distributed keys (The lines for BST, 4-ST, AVL and SL overlap.)

\section{CONCLUSION}

Our algorithms can also be used to store unbounded length strings. One approach would be to append $\$$ to the end of each string. To encode a binary string, 0,1 and $\$$ can be represented by 01,10 and 11 . Then, every encoded key is greater than 00 and smaller than 111 , so 00 and 111 can be used as keys of the two dummy nodes. With this modifications, searches would be non-blocking but not wait-free. Moreover, since labels of nodes never change, they need not fit in a dingle word.

The approach used in the replace operation can be used for operations on other data structures that must change several pointers atomically. Future work includes providing the general framework for doing this on any tree-based structure. Such a framework would have to guarantee that all changes become visible to query operations at the same time. Brown et al. [7] proposed a general technique for non-blocking trees that support one change to the tree atomically.

Since our algorithms create many Flag objects to avoid using locks, finding more efficient memory management techniques is an important area for future work.

Acknowledgments. I thank my supervisor, Eric Ruppert for his guidance and support, Trevor Brown for providing lots of help and code for the experiments and Michael L. Scott for giving us access to his multicore machines.

\section{REFERENCES}

[1] Y. Afek, H. Kaplan, B. Korenfeld, A. Morrison, and R. E. Tarjan. CBTree: A Practical Concurrent Self-Adjusting Search Tree. In Proc. Intl Symp. on Distributed Computing, pages 1-15, 2012.
[2] W. G. Aref and I. F. Ilyas. SP-GiST: An extensible database index for supporting space partitioning trees. J. Intel. Inf. Syst., pages 215-240, 2001.

[3] G. Barnes. A method for implementing lock-free shared-data structures. In Proc. ACM Symp. on Parallel Algorithms and Architectures, pages 261-270, 1993.

[4] P. Bieganski, J. Riedl, J. V. Carlis, and E. F. Retzel. Generalized suffix trees for biological sequence data: Applications and implementation. In Proc. Hawaii Intl Conf. on Syst. Sciences, pages 35-44, 1994.

[5] A. Braginsky and E. Petrank. A lock-free b+tree. In Proc. ACM Symp. on Parallelism in Algorithms and Architectures, pages 5867, 2012.

[6] N. G. Bronson, J. Casper, H. Chafi, and K. Olukotun. A practical concurrent binary search tree. In Proc. ACM Symp. on Principles and Practice of Parallel Programming, pages 257-268, 2010.

[7] T. Brown, F. Ellen, and E. Ruppert. A general technique for non-blocking trees. Manuscript, 2013.

[8] T. Brown and J. Helga. Non-blocking k-ary search trees. In Proc. Intl Conf. on Principles of Distributed Syst., pages 207221, 2011.

[9] D. Cederman and P. Tsigas. Supporting lock-free composition of concurrent data objects. In Proc. ACM Intl Conf. on Computing Frontiers, pages 53-62, 2010.

[10] F. Ellen, P. Fatourou, E. Kosmas, A. Milani, and C. Travers. Universal constructions that ensure disjoint-access parallelism and wait-freedom. In Proc. ACM Symp. on Principles of Distributed Computing, pages 115-124, 2012.

[11] F. Ellen, P. Fatourou, E. Ruppert, and F. van Breugel. Nonblocking binary search trees. In Proc. ACM Symp. on Principles of Distributed Computing, pages 131-140, 2010.

[12] M. Fomitchev and E. Ruppert. Lock-free linked lists and skip lists. In Proc. ACM Symp. on Principles of Distributed Computing, pages 50-59, 2004.

[13] K. Fraser. Practical lock freedom. PhD thesis, Cambridge University Computer Laboratory, 2003.

[14] M. Gan, M. Zhang, and S. Wang. Extended negative association rules and the corresponding mining algorithm. In Proc. Intl Conf. on Advances in Machine Learning and Cybernetics, pages 159168, 2006.

[15] M. Goodchild. Geographic information systems. Research Methods in Geography, pages 376-91, 2010.

[16] M. R. Guthaus, J. S. Ringenberg, D. Ernst, T. M. Austin, T. Mudge, and R. B. Brown. Mibench: A free, commercially representative embedded benchmark suite. In Proc. Workload Characterization, IEEE Intl Workshop, pages 3-14, 2001.

[17] T. Harris, J. Larus, and R. Rajwar. Transactional Memory, 2nd Edition. Morgan and Claypool Publishers, 2010.

[18] T. L. Harris. A pragmatic implementation of non-blocking linkedlists. In Proc. Intl Symp. on Distributed Computing, pages 300314, 2001.

[19] M. P. Herlihy and J. M. Wing. Linearizability: a correctness condition for concurrent objects. ACM Trans. Program. Lang. Syst., pages 463-492, 1990.

[20] S. V. Howley and J. Jones. A non-blocking internal binary search tree. In Proc. ACM Symp. on Parallelism in Algorithms and Architectures, pages 161-171, 2012.

[21] S. M. Z. Iqbal, Y. Liang, and H. Grahn. Parmibench - an open-source benchmark for embedded multiprocessor systems. Computer Architecture Letters, (2):45-48, 2010.

[22] D. R. Morrison. Patricia - practical algorithm to retrieve information coded in alphanumeric. J. ACM, pages 514-534.

[23] A. Pietracaprina and D. Zandolin. Mining frequent itemsets using patricia tries. In Frequent Itemsets Mining Implem., 2003.

[24] A. Prokopec, N. G. Bronson, P. Bagwell, and M. Odersky. Concurrent tries with efficient non-blocking snapshots. In Proc. ACM Symp. on Principles and Practice of Parallel Programming, pages $151-160,2012$. 
[25] N. Shavit and D. Touitou. Software transactional memory. In Proc. ACM Symp. on Principles of Distributed Computing, pages 204-213, 1995.

[26] H. Sundell and P. Tsigas. Scalable and lock-free concurrent dictionaries. In Proc. ACM Symp. on Applied Computing, pages 1438-1445, 2004.

[27] J.-J. Tsay and H.-C. Li. Lock-free concurrent tree structures for multiprocessor systems. In Proc. Intl Conf. on Parallel and Distributed Systems, pages 544-549, 1994.

[28] P. Zijlstra and R. Hat. Concurrent pagecache. In Linux Symp., page 311, 2007. 


\section{APPENDIX}

\section{A. Preconditions and Basic Invariants}

In this section, we show that the algorithms satisfy some basic invariants. First, we have the following observations from the pseudo-code.

Observation 1. The label field of a Node is never changed. No field of an Info object is changed except the state field.

Observation 2. The root pointer is never changed and root.label is $\varepsilon$.

Now, we prove that the precondition of the help routine is satisfied.

Lemma 3. Each call to the help routine satisfies its precondition.

Proof: We show that, for each call to help $(I), I$ is a Flag object. Just before calling help $(I)$ at line 110 or 119 . the operation checks that $I$ is a Flag object. Update operations call help $(I)$ at line 32, 41 or 71 just after creating a new Flag object $I$.

A CAS step that tries to change the child field of an internal node at line 98 inside help $(I)$ is called a child CAS of $I$.

Next, we show that each internal node has two non-null children, non-null values are passed to createNode and node is set to non-null value during the search operation.

Lemma 4. 1) Every internal node has two non-null children.

2) Every call to createNode satisfies its pre-condition.

3) Any process executing the loop of search has a non-null value in its node variable.

4) If a search returns $\langle g p, p$, node, -, -, - $\rangle$, then $p$ is an internal node and if gp is not null, gp is an internal node.

Proof: Assume the lemma is true before the step at time $T$. We shall prove the lemma is true after that step.

(1) We show that Claim 1 is true if a new internal node is created at $T$ or if a child field is changed at $T$. First, suppose a new internal node is created at time $T$. We show that the internal node that is created at $T$ has two non-null children. A new internal node is created at line 19, 121, 26 or 52 If a new internal node is created at line 19 at time $T$, the children of the new node are set to two new nodes that are created at that line. If a new copy of an internal node is made at line 26 or 52 at time $T$, since the lemma is true at all times before $T$, a new copy of some non-null node is made at $T$ and the children of the new copy of the node are set to two non-null nodes at $T$. If a new node is created at line 121 inside createNode $\left(\right.$ node $_{1}$, node $\left._{2}\right)$, since the invariant is true before $T$, node $_{1}$ and node $_{2}$ are non-null nodes and the children of the new node are set to node ${ }_{1}$ and node $_{2}$ at $T$.

Now, suppose the child field of an internal node is changed at $T$. The child field of an internal node is changed only at line 98. Let $I$ be a Flag object such that a child CAS of $I$ is executed at $T$. We consider each way that $I$ could have been created. For each case, we show that, for all $i$, I.newChild $[i]$ is a non-null node.

If $I$ is created at line 30, 31, 55, 57, 64 or 70, I.new Node[0] is set to a non-null node that is created at line 121 inside createNode, which is called at line 27, 53, 62 or 69 .

If $I$ is created at line 59. I.newNode[0] is set to a non-null node that is created at that line.

If $i=0$ and $I$ is created at line 40 or $i=1$ and $I$ is created at line 55 or 57 , let $\langle-, p,-,-,-,-\rangle$ be the result returned by the preceding call to $\operatorname{search}(\mathrm{val})$ on line 36 or 47 . Then, I.newNode[0] is set to a child of $p$ that is read at line 38 or 50 before $T$. Since the lemma is true before $T$, I.newNode[0] is set to a non-null node.

(2) Suppose createNode $\left(\right.$ node $_{1}$, node $\left._{2}\right)$ is called at $T$ from line 27, 53, 62, 67 or 69. We show that node $e_{1}$ and node $_{2}$ are non-null.

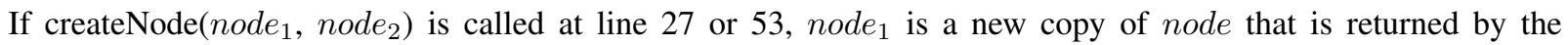
search operation at line 23 or 47 . Since the lemma is true prior to $T$, node $e_{1}$ is non-null. Then, node $_{2}$ is a new leaf node that is created at line 27 or 53 .

If createNode $\left(\right.$ node $_{1}$, node $\left._{2}\right)$ is called at line 62, let $\langle-, p,-,-,-,-\rangle$ be the result returned by the call to $\operatorname{search}(\mathrm{val})$ on line 47. Then, node $e_{2}$ is a new leaf node that is created at line 62 and node $e_{1}$ is a child of $p$ that is read at line 50 before $T$. Since the lemma is true before $T$, node $_{1}$ is a non-null node. 
If createNode $\left(\right.$ node $_{1}$, node $\left._{2}\right)$ is called at line 67, let $\langle g p, p,-,-,-,-\rangle$ be the result returned by the call to $\operatorname{search}(\mathrm{val})$ on line 47 Then, node 1 is a child of $p$ that is read at line 50 and node $_{2}$ is a child of $g p$ that is read at line 66 Since the lemma is true before $T$, node $e_{1}$ and node $_{2}$ are non-null nodes.

If createNode $\left(\right.$ node $_{1}$, node $\left._{2}\right)$ is called at line 69. node $e_{1}$ is a new node that is created at line 67 and node $_{2}$ is a new leaf node that is created at line 69.

(3) Before entering the loop of the search routines, node is set to root, which is not null. We must show that if node is set on line 82 at $T$, it is non-null. Since node is set to a child of a node at $T$ and the lemma is true prior to $T$, node is set to a non-null node at $T$.

(4) During the search operation, node is initialized to root at line 78, so at least one iteration of the loop is performed (since root is an internal node and root.label $=\varepsilon$ by Observation 2). By the test at line 79, $p$ is an internal node after every execution of line 81 .

In the first loop iteration, $g p$ is set to null. In each subsequent iteration, $g p$ is set to an internal node at line 80 (since $p$ is always an internal node after each execution of line 81).

By Lemma 4, we have the following observation.

Observation 5. Let $\left\langle g p_{d},-,-,-,-,-,\right\rangle$ be the result returned by a call to the search operation on line 45 and $\langle-,-$, node $\left._{i},-,-,-\right\rangle$ be the result returned by another call to the search operation on line 47 If gp $_{d}=$ node $_{i}$, then node ${ }_{i}$ is an internal node.

We use $a \cdot b$ to denote the concatenation of $a$ and $b$. Now, we show that the search operation satisfies some of its post-conditions if it returns.

Lemma 6. Assume search(val) returns $\langle g p$, $p$, node, gpInfo, $p I n f o, r m v d\rangle$. The following statements are true.

1) If gp is not null, then, at some time during the search operation, gp.info was gpInfo, and at some later time during the search operation, $p$ was a child of gp.

2) Then, at some later time during the search operation, p.info was pInfo, and at some later time during the search operation, p.child $[i]=$ node for some $i$.

3) (p.label) $\cdot i$ is a prefix of val.

4) If node is an internal node, node.label is not a prefix of val.

Proof: During the search operation, node is initialized to root at line 78, so at least one iteration of the loop is performed (since root is an internal node and root.label $=\varepsilon$ by Observation 2).

1) Assume $g p$ is not null. By Lemma 4, $g p$ is an internal node. Since $g p$ is set to null during the first loop iteration, the search operation does not exit the loop after the first loop iteration. During the second last loop iteration, $p I n f o$ and $p$ are set at line 81 and node is set to a child of $p$ at line 82 . Then, during the last loop iteration, gpInfo and $g p$ are set to $p I n f o$ and $p$ at line 80 and $p$ is set to node at line 81 . So, when line 81 was executed for the second last time, gp.info $=g p \operatorname{Inf} o$ and then, when line 82 was executed for the second last time, $p$ was a child of $g p$.

2) By Lemma 4, $p$ is an internal node. During the last loop iteration, $p$ Info and $p$ are set at line 81 and node is set to a child of $p$ at line 82 So, when line 81 was executed for the last time, p.info $=p \operatorname{Info}$ and then, when line 82 was executed for the last time, p.child $[i]=$ node for some $i$.

3) During the last loop iteration, $p$ is set to node at line 81 . Since the condition at line 79 is true at the beginning of that iteration, $p$ is set to an internal node whose label is a prefix of val. At the last execution of line 82 . node is set to p.child $[i]$ such that $i=(|p . l a b e l|+1)$ th bit of val. So, $(p . l a b e l) \cdot i$ is a prefix of val.

4) Since the search operation exits the loop, the condition is false at the last execution of line 79 . If node is an internal node, node.label is not a prefix of val.

Next, we show that, just after a child field is set, the following Patricia trie property is preserved: if $x . \operatorname{child}[i]=y$, then $(x . l a b e l) \cdot i$ is a prefix of y.label.

Invariant 7. Let $x$ be an internal node and $y=x$.child $[i]$. Then, $(x . l a b e l) \cdot i$ is a prefix of y.label.

Proof: By Observation 1, no label field of a node is ever changed. So, we need only show that the lines 19 . 26. 121 and 52, which create new internal nodes, and line 98, which changes the child field of an internal node, 
preserve the invariant. We assume the invariant holds at all times prior to $T$ and we prove that the invariant is true just after $T$. First, we show if a new internal node is created at $T$, the invariant is preserved.

Line 19 creates a new internal node root that has two children. Initially, root.label $=\varepsilon$, root.child $[0]$.label $=$ $000 \ldots 0$ and root.child[1].label $=111 \ldots 1$.

If a new copy of an internal node is created on line 26 or 52 at $T$, since the invariant is true at all times before $T$, the new copy of the node satisfies the invariant.

If a new node is created at line 121 inside createNode, its label is the longest common prefix of the label fields of its children. Since the two children's labels are not prefixes of each other, the invariant is true.

In the remainder of the proof, we show if a child CAS of $I$ succeeds at $T$, the invariant is preserved. The child CAS changes I.pNode[j].child $[i]$ from I.oldChild $[j]$ to I.newChild $[j]$ (for some $j$ ) where $i$ is the $(|I . p N o d e[j] . l a b e l+1|)$ th bit of I.newChild $[j] . l a b e l$. Thus, it suffices to show that I.pNode $[j] . l a b e l$ is a proper prefix of I.newChild[j].label. We consider each way that $I$ could have been created. By Observation 1 , after the initialization of $I$, no field of $I$ is changed except the flagDone field.

Case 1: $I$ is created at line 30 or 31 Let $\langle-, p$, node,,,---$\rangle$ be the result returned by the call to $\operatorname{search}($ val $)$ on line 23 that precedes the creation of $I$. Then, newNode is the new node that is created at line 27 and whose children are a new copy of node and a new leaf node whose label is val. In this case, I.pNode $[0]=p$ and I.newChild $[0]=$ newNode. By Lemma 6, p.child $[k]=$ node for some $k$ at some time before $T$. Since the invariant is true before $T,(p$.label $) \cdot k$ is a prefix of node.label. By Lemma 6, (p.label) $k$ is a prefix of val. Since newNode.label is the longest common prefix of val and node.label, $(p . l a b e l) \cdot k$ is a prefix of newNode.label.

Case 2: $I$ is created at line 40 Let $\langle g p, p$, node, -, -, - $\rangle$ be the result returned by the call to search $(v a l)$ on line 36 that precedes the creation of $I$. Let nodeSibling be the child of $p$ that is read at line 38 Since the invariant is true before $T$, p.label is a prefix of nodeSibling.label. In this case, I.pNode $[0]=$ gp, I.oldChild $[0]=p$, and I.newChild $[0]=$ nodeSibling. Since the child CAS succeeds at $T$, gp.child $[i]$ was $p$ just before T. Since the invariant is true before $T$, (gp.label) $\cdot i$ is a prefix of p.label. Since p.label is a prefix of nodeSibling.label, (gp.label) $\cdot i$ is a prefix of nodeSibling.label.

Case 3: $I$ is created at line 55 or 57 Let $\left\langle g p_{d}, p_{d}\right.$, node $\left.e_{d},-,-,-\right\rangle$ and $\left\langle-, p_{i}\right.$, node $\left._{i},-,-,-\right\rangle$ be the results returned by the calls to the search routine on line 45 and 47 that precede the creation of $I$. Then, I.pNode $[0]=p_{i}$ and I.newChild $[0]$ is the new node that is created at line 53 By the same argument as in Case 1, the invariant is true if a child CAS of $I$ on I.pNode[0] succeeds at T. Let nodeSibling be the child of $p_{d}$ that is read on line 50 Then, I.pNode[1] $=g p_{d}$ and I.newChild[1] = nodeSibling. By the same argument as in Case 2, the invariant is true if a child CAS of $I$ on I.pNode[1] succeeds at $T$.

Case 4: $I$ is created at line 59 Let $\left\langle-, p_{i}\right.$, node $\left._{i},-,-,-\right\rangle$ be the result returned by the call to $\operatorname{search}\left(v a l_{i}\right)$ on line 47 that precedes the creation of $I$. In this case, I.pNode $[0]=p_{i}$ and I.newChild[0] is a new leaf node whose label is $v_{a l}$. By Lemma 6, $p_{i}$.label is a proper prefix of $v^{2} l_{i}$.

Case 5: $I$ is created at line 64. Let $\left\langle g p_{d}, p_{d}\right.$, node $\left.e_{d},-,-,-\right\rangle$ and $\left\langle-, p_{i}\right.$, node $\left._{i},-,-,-\right\rangle$ be the results returned by the calls to the search routine on line 45 and 47 that precede the creation of $I$. Let nodeSibling be the child of $p_{d}$ that is read at line 50 and newNode be the new node that is created at line 62 and whose children are nodeSibling and a new leaf node whose label is val $_{i}$. In this case, I.pNode $[0]=p_{i}$, I.oldChild $[0]=$ node $_{i}$ and I.newChild $[0]=$ newNode. Since the child CAS succeeds at $T$, gp $p_{d}$.child $[i]$ was $p_{d}$ just before $T$. Since the invariant is true before $T,\left(g p_{d} . l a b e l\right) \cdot i$ is a prefix of $p_{d}$.label. Since the invariant is true before $T$ and nodeSibling is a child of $p_{d}$ before $T, p_{d}$.label is a prefix of nodeSibling.label. So, $\left(g p_{d} . l a b e l\right) \cdot i$ is a prefix of nodeSibling.label.

If the condition at line 60 is true, by Lemma 6, $\left(g p_{d} . l a b e l\right) \cdot i$ is a prefix of $v a l_{i}\left(\right.$ since $p_{i}=g p_{d}$ and $n o d e_{i}=p_{d}$ ). If the condition at line 61 is true, by Lemma 6, $p_{i}$.label is a prefix of $v a l_{i}$, so $\left(g p_{d} . l a b e l\right) \cdot i$ is a prefix of $v a l_{i}$ (since $\left.p_{i}=p_{d}\right)$. Since the children of newNode are nodeSibling and a new leaf node whose label is $v a l_{i},\left(g p_{d} . l a b e l\right) \cdot i$ is a prefix of newNode.label.

Case 6: $I$ is created at line 70 Let $\left\langle g p_{d}, p_{d}\right.$, node $\left._{d},-,-,-\right\rangle$ and $\left\langle-, p_{i}\right.$, node $\left._{i},-,-,-\right\rangle$ be the results returned by the calls to the search routine on line 45 and 47 that precede the creation of $I$. Let nodeSibling be the child of $p_{d}$ that is read at line 50 and $p$ Sibling be the child of $g p_{d}$ that is read at line 66 . Then, newChild is the new node that is created at line 67 and whose children are nodeSibling and pSibling. Then, newNode is the new node that is created at line 69 and whose children are newChild and a new leaf node whose label is val $_{i}$. In this case, node $_{i}=g p_{d}, I . p N o d e[0]=p_{i}$, I.oldChild $[0]=$ node $_{i}$ and I.newChild $[0]=$ newNode. 
By Lemma 6, $p_{d}$ was a child of $g p_{d}=$ node $_{i}$ at some time before $T$. Since the invariant is true before $T$, node $e_{i}$ label is a prefix of $p_{d}$.label. Since the invariant is true before $T$ and nodeSibling is a child of $p_{d}$ before $T$, $p_{d}$. label is a prefix of nodeSibling.label. So, node $e_{i}$.label is a prefix of nodeSibling.label. Since the child CAS succeeds at $T, p_{i}$.child $[i]=$ node $_{i}$ just before $T$. Since the invariant is true before $T,\left(p_{i} . l a b e l\right) \cdot i$ is a prefix of

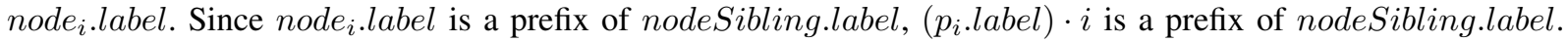

Since the invariant is true before $T$ and $p$ Sibling is a child of $g p_{d}=$ node $_{i}$ before $T$, node $e_{i}$.label is a prefix of $p$ Sibling.label. Since $\left(p_{i}\right.$. label $) \cdot i$ is a prefix of node $i_{i}$ label, $\left(p_{i}\right.$.label $) \cdot i$ is a prefix of $p$ Sibling.label. Since the children of newChild are nodeSibling and pSibling, $\left(p_{i}\right.$. label $) \cdot i$ is a prefix of newChild.label.

By Lemma 6. $\left(p_{i} . l a b e l\right) \cdot i$ is a prefix of $v_{a l}$. Since the children of newNode are newChild and a new leaf node whose label is $\mathrm{val}_{i},\left(p_{i}\right.$. label $) \cdot i$ is a prefix of new Node.label.

We say that an internal node pNode is a parent of a node $c N$ ode at time $T$, if $c N o d e$ is an element of $p N o d e . c h i l d$ at time $T$. Note that a node $c N$ ode may have more than one parent at time $T$. (For example, this occurs if $c$ Node's parent has been removed from the tree but still has a child pointer to $c$ Node.)

We say that a node anc is an ancestor of a node $x$ at time $T$ if there is a path consisting of child pointers from anc to $x$ at time $T$. We say that node $x$ is a descendant of anc at time $T$. If anc $\neq x$, we say anc is a proper ancestor of a node $x$ at time $T$ and $x$ is a proper descendant of a node anc at time $T$. We say a node $x$ is reachable at time $T$ if root is an ancestor of $x$ at time $T$. 7.

The root cannot have a parent $x$ because $x$.label would have to be a proper prefix of root.label $=\varepsilon$ by Invariant

Corollary 8. The root does not have any parent at any time.

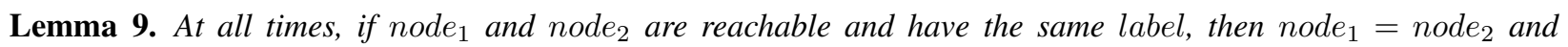
there is exactly one reachable parent of node (unless node $_{1}$ is the root).

Proof: Let $v_{0}, v_{1}, \ldots, v_{n-1}, v_{n}$ be a path consisting of child pointers at time $T$ such that $v_{0}=$ root and

$v_{n}=$ node $_{1}$ and $v_{0}^{\prime}, v_{1}^{\prime}, \ldots, v_{m-1}^{\prime}, v_{m}^{\prime}$ be a path consisting of child pointers at time $T$ such that $v_{0}^{\prime}=$ root and $v_{n}^{\prime}=$ node $_{2}$. Without loss of generality, assume $n \leq m$.

By induction, we show these two paths are the same. Since $v_{0}=\operatorname{root}$ and $v_{0}^{\prime}=\operatorname{root}$, $v_{0}=v_{0}^{\prime}$. Assuming $v_{i-1}=v_{i-1}^{\prime}$, we show that $v_{i}=v_{i}^{\prime}$. By Invariant 7. $\left(v_{i-1}\right.$. label $) \cdot j$ is a prefix of node .label $^{\prime}=$ node $e_{2}$. label

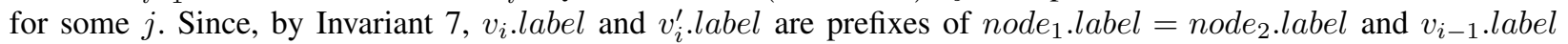
is a proper prefix of $v_{i}$.label and $v_{i}^{\prime}$ label, $\left(v_{i-1}\right.$.label $) \cdot j$ is a prefix of $v_{i}$.label and $v_{i}^{\prime}$.label. By Invariant 7 . $v_{i-1} \cdot \operatorname{child}[j]=v_{i}$ at time $T$ and $v_{i-1} \cdot \operatorname{child}[j]=v_{i}^{\prime}$ at time $T$, so $v_{i}=v_{i}^{\prime}$.

So, node $_{1}=v_{n}^{\prime}$. Then, $n=m$ since $v_{n}^{\prime}$.label is not a proper prefix of node $e_{2}$.label. Therefore, $v_{n}=v_{m}^{\prime}$ and node $_{1}=$ node $_{2}$.

Since $v_{n-1}=v_{m-1}^{\prime}$, if node $1 \neq$ root, there is exactly one reachable parent of node $_{1}$ at time $T$.

\section{B. Behaviour of CAS Steps on info Fields}

In this section, we show how CAS steps change the info field of nodes. First, we define flagging and unflagging of nodes formally.

Definition 10. Let $x$ be a node. If $x$.info is a Flag object at time $T$, we say $x$ is flagged at time T. The CAS step at line 90 is called a flag CAS.

Definition 11. Let $x$ be an internal node. If $x$.info is an Unflag object at time $T$, we say $x$ is unflagged at time T. The CAS step at line 101 is called an unflag CAS. The CAS step at line 105 is called a backtrack CAS.

All nodes are unflagged when they are created. A leaf node can only become flagged at line 95. Once a leaf node is flagged, it can never become unflagged. The following lemma describes how the info field of an internal node is initialized and changed when the node becomes flagged or unflagged.

Lemma 12. Let $x$ be an internal node. When node $x$ is created, x.info is initially set to a new Unflag object. The only changes to $x$.info that can occur are (1) a flag CAS at line 90 that changes $x$.info from an Unflag object to a Flag object, or (2) an unflag CAS at line 101 or a backtrack CAS at line 105 that changes x.info from a Flag object to a newly created Unflag object. 
Proof: A new internal node is only created at line 19, 26, 121 or 52 and the info field of the new internal node is initially set to a new Unflag object. The only lines that can change $x$.info are line 90,101 and 105

Suppose a flag CAS at line 90 inside help $(I)$ succeeds to change $x$.info from an element of I.oldInfo to $I$. By Lemma 3, $I$ is a Flag object. Before creating $I$, the operation checks that each value stored in I.oldInfo is an Unflag object at line 109

Suppose an unflag or backtrack CAS inside help $(I)$ succeeds to change $x$.info. By Lemma $3, I$ is a Flag object. So, x.info was equal to $I$ just before the unflag or backtrack CAS and, just after the unflag or backtrack CAS, $x$.info is changed to a newly created Unflag object.

Let $I$ be a Flag object. Elements of $I$.flag are ordered at line 115 before $I$ is created. Let $F_{I}$ be the set of all nodes in the I.flag array and $U_{I}$ be the set of all nodes in the I.unflag array. By the pseudo-code, $U_{I} \subseteq F_{I}$.

A flag, unflag or backtrack CAS step executed inside help $(I)$ is called a CAS step of $I$. A flag CAS of $I$ attempts to change the info field of an internal node in $F_{I}$ from an Unflag object to $I$. An unflag CAS of $I$ attempts to change the info field of an internal node in $U_{I}$ from $I$ to a new Unflag object. A backtrack CAS of $I$ attempts to change the info field of an internal node in $F_{I}$ from $I$ to a new Unflag object.

By Lemma 12, we have the following corollary.

Corollary 13. Let I be a Flag object. After an internal node $x$ is flagged by a flag CAS of $I$, the next change to x.info can only be done by an unflag or backtrack CAS of I.

Let $I$ be a Flag object. A flag CAS of $I$ at line 90 inside help $(I)$ tries to change I.flag $[i]$.info from I.oldInfo[i] to $I$ for some $i$. The following lemma shows that I. flag $[i]$.info was equal to I.oldInfo $[i]$ at some time before $I$ was created.

Lemma 14. Let I be a Flag object. Then, each entry of I.flag[i] is an internal node. Also, if $x=I$.flag[i] (for some $i$ ), then $x$.info was I.oldInfo $[i]$ at some time before I was created.

Proof: Just before creating $I$, an update operation calls search once (for insert and delete) or twice (for replace). If one such search returns $\langle g p, p$, node, gpInfo, pInfo, - $\rangle$, then I.flag[i] is set to $p, g p$ or node at line 30,31 . 40, 55, 57, 59,64 or 70.

By Lemma 4, $p$ is an internal node and $g p$ is an internal node if $g p$ is not null. If $I$.flag[i] is set to $g p$ at line 40,55, 57, 64 $g p$ is not null by the pseudo-code. If $I . f l a g[i]$ is set to $g p$ at line 70, $g p$ is not null by Observation 5. When I. flag $[i]$ is set to $p$ or $g p$ at line 30, 31, 40, 55, 57, 59, 64 or 70, I.oldInfo[i] is set to the corresponding pInfo or gpInfo. By Lemma 6, I.flag[i].info =I.oldInfo[i] at some time during search(val).

If I.flag [i] is set to node at line 30 or 55 node is an internal node. Then, I.oldInfo $[i]$ is set to a value read from node.info at line 25 or 49 .

Suppose some process reads a value old in some variable $x$ at time $T$ and then later performs a CAS that changes $x$ from old to new at time $T^{\prime}$. Some of the later parts of our proof of correctness will rely on the fact that $x$ has not been changed from old between $T$ and $T^{\prime}$. If $x$ is changed between $T$ and $T^{\prime}$ and $x$ is changed back to old just before $T^{\prime}$, the CAS step would incorrectly change $x$ to new. This situation is called the ABA problem. Since CAS steps are used to change the info and child fields of internal nodes, we show that the ABA problem is avoided on the info and child fields of internal nodes. First, we show that the info field of an internal node is not set to a value that it has had previously.

Lemma 15. Let $x$ be an internal node. Then, x.info is never set to a value that it has had previously.

Proof: Assume $x$.info is set to new at time $T$. We show that $x$.info $\neq$ new at all times before $T$. First, consider the case where new is an Unflag object. By Lemma 12, only the CAS steps at line 101 and 105 change $x . i n f o$ to an Unflag object. Since, at those lines, the CAS step changes $x$.info to a newly created Unflag object, $x$. info is never set at line 101 or 105 to a value that it has had previously.

Now, consider the case where new is a Flag object. By Lemma 12, only the CAS step at line 90 inside help(new) tries to change $x$.info to new. To derive a contradiction, assume the lemma is violated for the first time at time $T$. Then, $x$.info is set to new at time $T$ and $x . i n f o$ had value new at some time before $T$. Since $x$.info is initially an Unflag object when $x$ is created, there must exist a CAS step that sets $x$.info to new at some time $T^{\prime}$ before time $T$. Let $i_{1}$ and $i_{2}$ be the values of $i$ when a process executes line 90 at $T^{\prime}$ and $T$. Then, new.flag $\left[i_{1}\right]=x$ and new.flag $\left[i_{2}\right]=x$. Since only one copy of duplicate elements of new.flag is kept at line $114, i_{1}=i_{2}$. Let old be 
new.oldInfo $\left[i_{1}\right]=$ new.oldInfo $\left[i_{2}\right]$. At time $T^{\prime}, x$. info is changed from old to new. Then, at time $T$, x.info is changed from old to new. Thus, x.info is set to old again between $T^{\prime}$ and $T$, contradicting the assumption that the lemma is violated for the first time at time $T$.

So, by Lemma 15, if a CAS step succeeds to change the info field of an internal node from some old value to some new value, the info field of the internal node has not been changed since the time that the value of the info field of the internal node was read as the old value. Thus, the ABA problem on the info fields of internal nodes is avoided.

In the Patricia trie implementation, update operations might help one another to flag and unflag nodes by calling the help routine at line 110 . So, there might be several CAS steps that try to change the info field of some internal node from a value old to some value new. We show only the first CAS step among the CAS steps of this group can succeed to change the info field of the internal node from old to new.

Lemma 16. Let $x$ be an internal node. Assume a group of CAS steps tries to change x.info from old to new. Only the first CAS step in this group might succeed.

Proof: Assume a group of CAS steps each tries to change x.info from old to new. By Lemma 12, no two CAS steps can try to change $x$.info to the same Unflag object. So, new must be a Flag object. Thus, the CAS steps that try to change $x$.info are Flag CASs inside calls to help(new). So, there is some $i$ such that new.flag $[i]=x$ and new.oldInfo $[i]=$ old. By Lemma 14, $x$. info $=$ old at some time before new is created. So, all CAS steps that try to change $x$.info from old to new occur after a time when the value of $x$.info is old. After a CAS step changes $x$.info from old to some value, by Lemma 15, x.info is not set to old again. If the first CAS step among the CAS steps in the group changes x.info from old to new, no other CAS step in the group can change x.info from old to new. If the first CAS step among the CAS steps in the group does not change $x$.info from old to new, x.info has already been changed from old to some other value and no other CAS step in the group can change $x$.info from old to new.

Let $I$ be a Flag object. Recall that $F_{I}$ is the set of all nodes in the I.flag array. To avoid live-lock in flagging internal nodes, all operations try to flag internal nodes in some defined order. Before creating $I$, elements of $F_{I}$ are sorted at line 115. We show that if elements of $F_{I}$ are successfully flagged by flag CAS steps of $I$, they are flagged in order.

Lemma 17. Let $I$ be a Flag object and let $f$ be the number of of entries in I.flag. For $0 \leq i<f-1$, a flag CAS of I does not successfully flag I.flag $[i+1]$ unless I.flag[i] is flagged earlier by a flag CAS of I.

Proof: Assume a help routine tries to flag I.flag $[i+1]$ using a flag CAS of $I$. Then, the $\operatorname{doChildCAS}$ variable is true at the previous execution of line 91. After the help routine tries to flag a node, doChildCAS is set to false at line 91 if the info field of the node is not $I$. So, the help routine tries to flag I.flag $[i+1]$ using a flag CAS of $I$ only if $I . f l a g[i]$.info was $I$. Thus, by Lemma 12 I.flag $[i]$ has already been flagged by a flag CAS of $I$.

If I.flagDone is true at line 99, help $(I)$ performs unflag CAS steps on the nodes in $U_{I}$ at line 101. Otherwise, help $(I)$ performs backtrack CAS steps on the nodes in $F_{I}$ at line 105 . The following lemma shows that I.flagDone is set to true at line 94 only after all nodes in $F_{I}$ get flagged successfully by flag CAS steps of $I$.

Lemma 18. Let I be a Flag object. Before I.flagDone is set to true at line 94 all nodes in $F_{I}$ are flagged by flag CAS steps of $I$.

Proof: The help routine sets I. flagDone to true at line 94 only if the $\operatorname{doChildCAS}$ variable is true at line 93 If the info field of $I . f l a g[i] \neq I$ for any $i$, doChildCAS gets set to false at line 91 and the loop terminates. So, doChildCAS is true at line 93 only if all nodes in $F_{I}$ were flagged successfully using flag CAS steps of $I$.

From the pseudo-code, we have the following observations.

Observation 19. Let I be a Flag object. I.flagDone is initially false and I.flagDone is only set to true.

Observation 20. Let I be a Flag object. Then, I.flagDone is set to true before any child CAS step of I occurs.

We wish to show that the child field of an internal node is changed by a child CAS of $I$ at line 98 only while all internal nodes in $F_{I}$ are flagged by $I$. First, we show a child CAS of $I$ is executed only after all nodes in $F_{I}$ get flagged using flag CAS steps of $I$. Then, we show that, for all $j$, there is no successful unflag or backtrack 
CAS of $I$ before the first child CAS of $I$ on I.pNode $[j]$.

Lemma 21. Let I be a Flag object. A child CAS step of I is preceded by flagging all nodes in $F_{I}$ using flag CAS steps of $I$.

Proof: By Observation 20, a child CAS step of $I$ can be executed only after I.flagDone is set to true. By Lemma 18 , setting I.flagDone to true is preceded by flagging all nodes in $F_{I}$ using flag CAS steps of $I$.

Lemma 22. Let I be a Flag object. If there is any child CAS of I, then for all $j$, there is no unflag or backtrack CAS of I before the first child CAS step of I on I.pNode $[j]$.

Proof: We prove the lemma by contradiction. Assume that for some $j$, the first child CAS of $I$ on $I . p N o d e[j]$ is at time $T$ and an invocation $H$ of help $(I)$ executes the first unflag or backtrack CAS of $I$ at $T^{\prime}$ before $T$. Since the first child CAS of $I$ on $I . p N o d e[j]$ is at $T, H$ does not execute any child CAS of $I$ on $I . p N o d e[j]$ at line 98 before $T^{\prime}$. So, the doChildCAS variable is false when $H$ performs line 93 before $T^{\prime}$. Thus, $H$ sets the doChildCAS variable to false at line 91 when y.info $\neq I$ just after $H$ tries to flag some node $y$ by a flag CAS of $I$. By Lemma 21, since $y \in F_{I}$, y.info is set to $I$ before $T$. By Lemma 16, only the first flag CAS of $I$ on y.info succeeds. Since a flag CAS of $I$ on y.info is performed just before the line 91, y.info is set to $I$ before $H$ executes line 91. Then, it is changed from $I$ to another value before $H$ reads $y$.info at line 91, contradicting the fact that the first unflag or backtrack CAS of $I$ is at time $T^{\prime}$.

Next, we show that there is no backtrack CAS of $I$ after the first child CAS of $I$. This will imply that nodes in $F_{I}-U_{I}$ remain flagged forever after a child CAS of $I$ occurs.

Lemma 23. Let I be a Flag object. If there is any child CAS of I, there is no backtrack CAS of I.

Proof: Assume the first child CAS of $I$ is at time T. By Lemma 22, there is no backtrack CAS of $I$ before $T$. To derive a contradiction, assume the first backtrack CAS of $I$ after $T$ is performed by some invocation $H$ of $\operatorname{help}(I)$.

Then, when $H$ checks I.flagDone at line 99. I.flagDone is false. By Observation 20, I.flagDone is set to true before $T$, so $H$ performs line 99 before $T$. Since $H$ does not set $I$. flagDone to true at line 94, $H$ must have set doChildCAS to false at line 91 after seeing y.info $\neq I$ for some node $y \in F_{I}$ at line 91. By Lemma 21. $y$.info is set to $I$ before $T$. By Lemma 16, the first flag CAS of $I$ on y.info succeeds. So, $y$.info is changed from $I$ to another value before $H$ reads y.info at line 91, which is prior to $T$ (since $H$ performs line 99 before $T)$. This contradicts Lemma 22.

By the pseudo-code, we have the following lemma.

Observation 24. Let I be a Flag object. For each i, I.pNode $[i] \in F_{I}$ and I.pNode $[i] \in U_{I}$.

Let $I$ be a Flag object and $x \in F_{I}$. The following lemma shows what the value of $x$.info is between the time when x.info is read during the search preceding the creation of $I$ and the successful child CAS of $I$.

Lemma 25. Let $I$ be a Flag object and $x=I$.flag $[i]$ and $x$ Info $=$ I.oldInfo[i] for some $i$. Suppose x.info $=$ $x$ Info at time $T_{1}$ and for some $j$ the first child CAS of I on I.pNode[j] occurs at time $T_{2}>T_{1}$. Then, at all times between $T_{1}$ and $T_{2}$, x.info is either xInfo or $I$.

Proof: By Lemma21, x.info is changed from $x$ Info to $I$ between $T_{1}$ and $T_{2}$. By Lemma 15, x.info $=x \operatorname{Info}$ at all times between $T_{1}$ and the time when $x$.info is set to $I$. By Lemma 15 and 22, $x$.Info $=I$ at all times between the time when $x$.info is set to $I$ and $T_{2}$.

Let $I$ be a Flag object that is created by a replace operation. Then, the leaf node I.rmvLeaf might be flagged at line 95 during help $(I)$. By Lemma 18 and the pseudo-code, we have the following observation.

Observation 26. Let I be a Flag object. Then, I.rmvLeaf is a leaf node if and only if I is created at line 55 or 57 For such Flag object I, setting I.rmvLeaf.info to I is preceded by flagging all nodes in $F_{I}$ using flag CASs of I and setting I.flagDone to true. Furthermore, a child CAS of I is preceded by setting I.rmvLeaf.info to I. After I.rmvLeaf is flagged, it never becomes unflagged.

\section{Behaviour of CAS Steps on child Fields}

In this section, we show how CAS steps change the child field of nodes. 
Just before creating a Flag object $I$, an update operation calls search once (for insert and delete) or twice (for replace). Then, for each $i, I . p N o d e[i]$ and I.oldChild $[i]$ are set to either $g p$ and $p$ or $p$ and node where $\langle g p, p$, node, -, -, - $\rangle$ is the result of one of these searches. So, by Lemma 6 , we have the following observation.

Observation 27. Let I be a Flag object. The values of I.pNode $[i]$ and I.oldChild[i] are returned by a call to the search operation that precedes the creation of $I$. At some point during that search operation, I.oldChild $[i]$ was a child of I.pNode $[i]$.

Next, we show that each successful child CAS changes the child field of an internal node from some old value to some new value that is different from the old value.

Lemma 28. Let I be a Flag object. Then for all $i$, I.oldChild $[i] \neq$ I.newChild $[i]$.

Proof: If $i=0$ and $I$ is created on line 30, 31, 55, 57, 59, 64 or 70 then I.newChild [i] is a newly created node, I.oldChild $[i]$ is set to node or $p$ where $\langle-, p$, node,,,---$\rangle$ was returned by a search operation that preceded the creation of $I$. By Lemma 6, I.oldChild $[i]$ was a child of some internal node during the search operation. I.newChild $[i]$ is created at line 121 or 59 after the search operation returns. So, I.oldChild $[i] \neq$ I.newChild $[i]$.

If $i=0$ and $I$ is created on line 40 or if $i=1$ and $I$ is created at line 55 or 57, then I.oldChild is set to $p$ and the value of I.newChild is read from p.child at line 38 or 50 . By Invariant 7, I.oldChild $[i] \neq$ I.newChild $[i]$.

The decision of which child to update is made at line 97 based on the (|I.pNode[i].label $\mid+1)$ th bit of I.newChild $[i]$.label. Now, we show the child CAS changes the same element of the child field that is read during the search operation.

Lemma 29. Let I be a Flag object. Then, for each $i,(|I . p N o d e[i] . l a b e l|+1)$ th bit of I.oldChild[i].label and I.newChild[i].label are the same.

Proof: Let $k$ be $\mid$ I.pNode $[i] . l a b e l \mid+1$. To prove the lemma, we consider different cases according to what line created $I$.

Case 1: $I$ is created at line 30 or 31 Let $\langle-, p$, node,,,---$\rangle$ be the result returned by the call to $\operatorname{search}(v a l)$ on line 23 that precedes the creation of $I$. Let newNode be the new internal node that is created at line 27. Then, I.pNode $[0]=p$, I.oldChild $[0]=$ node and I.newChild $[0]=$ newNode. By Lemma 6, p.child $[j]=$ node for some $j$ at some time during $\operatorname{search}(\mathrm{val})$ and $(\mathrm{p} \mathrm{label}) \cdot j$ is a prefix of val. By Invariant 7 , $(\mathrm{p}$.label $) \cdot j$ is also a prefix of node.label. So, the $k$ th bits of node.label and val are $j$. Initially, the children of newNode are a new leaf node whose label is $\mathrm{val}$ and a new copy of node. Since $($ p.label $) \cdot j$ is a prefix of node.label and val, the $k$ th bit of newNode.label is $j$.

Case 2: $I$ is created at line 40. Let $\langle g p, p$, node, -, -, - $\rangle$ be the result returned by the call to $\operatorname{search}(v a l)$ on line 36 that precedes the creation of $I$. Let nodeSibling be the element of p.child that is read at line 38 Then, I.pNode $[0]=$ gp, I.oldChild $[0]=p$ and I.newChild $[0]=$ nodeSibling. By Lemma 6, gp.child $[j]=p$ for some $j$ at some time during $\operatorname{search}(\mathrm{val})$. By Invariant 7. $(\mathrm{gp} . \mathrm{label}) \cdot j$ is a prefix of p.label and p.label is a prefix of nodeSibling.label. So, $k$ th bits of p.label and nodeSibling.label are $j$.

Case 3: $I$ is created at line 55 or 57 Let $\left\langle g p_{d}, p_{d}\right.$, node $\left.e_{d},-,-,-\right\rangle$ be the result returned by the call to $\operatorname{search}\left(v a l_{d}\right)$ on line 45 that precedes the creation of $I$ and $\left\langle-, p_{i}\right.$, node $\left._{i},-,-,-\right\rangle$ be the result returned by the call to $\operatorname{search}\left(v a l_{i}\right)$ on line 47 that precedes the creation of $I$. Then, new Node $i_{i}$ is the new internal node that is created at line 53 . Then, I.pNode $[0]=p_{i}$, I.oldChild $[0]=$ node $_{i}$ and I.newChild $[0]=$ newNode $_{i}$. By the same argument as in Case 1,

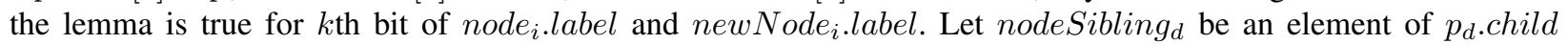
that is read at line 38 Then, I.pNode $[0]=g p_{d}$, I.oldChild $[0]=p_{d}$ and I.newChild $[0]=$ nodeSibling . By the same argument as in Case 2, the lemma is true for $k$ th bit of $p_{d}$.label and nodeSibling $g_{d}$ label.

Case 4: $I$ is created at line 59 Let $\left\langle-, p_{i}\right.$, node $\left._{i},-,-,-\right\rangle$ be the result returned by the call to $\operatorname{search}\left(v l_{i}\right)$ on line 47 that precedes the creation of I. Then, I.pNode $[0]=p_{i}$, I.oldChild $[0]=$ node $_{i}$ and I.newChild $[0]$ is the new leaf node whose label is $v a l_{i}$. By Lemma 6 $p_{i}$.child $[j]=$ node $e_{i}$ for some $j$ at some time during $\operatorname{search}\left(v_{a} l_{i}\right)$ and $\left(p_{i} . l a b e l\right) \cdot j$ is a prefix of $\operatorname{val}_{i}$. By Invariant 7. $\left(p_{i} . l a b e l\right) \cdot j$ is a prefix of node $e_{i}$ label.

Case 5: $I$ is created at line 64 Let $\left\langle g p_{d}, p_{d}\right.$, node $\left.e_{d},-,-,-\right\rangle$ be the result returned by the call to $\operatorname{search}\left(v a l_{d}\right)$ on line 45 that precedes the creation of $I$ and $\left\langle-, p_{i}\right.$, node $\left._{i},-,-,-\right\rangle$ be the result returned by the call to $\operatorname{search}\left(v^{2} l_{i}\right)$ on line 47 that precedes the creation of $I$. Then, newNode ${ }_{i}$ is the new internal node that is created at line 62 Then, I.pNode $[0]=g p_{d}$, I.oldChild $[0]=p_{d}$ and I.newChild $[0]=$ newNode $_{i}$. Let nodeSibling be $_{d}$ the child 
of $p_{d}$ that is read at line 50 By Lemma 6, gp $p_{d} \cdot \operatorname{child}[j]=p_{d}$ for some $j$ at some time during $\operatorname{search}\left(v a l_{d}\right)$. By Invariant 7. $\left(g p_{d} . l a b e l\right) \cdot j$ is a prefix of $p_{d}$.label and $p_{d}$.label is a prefix of nodeSibling $g_{d} . l a b e l$. So, $\left(g p_{d} . l a b e l\right) \cdot j$ is a prefix of nodeSibling.$l a b e l$ and the $k$ th bit of $p_{d} . l a b e l$ is $j$.

If the condition at line 60 is true, by Lemma 6. $\left(g p_{d} . l a b e l\right) \cdot j$ is a prefix of $v a l_{i}\left(\right.$ since $p_{i}=g p_{d}$ and $n o d e_{i}=p_{d}$ ). If the condition at line 61 is true, by Lemma 6, $p_{i}$.label is a prefix of $v a l_{i}$, so $\left(g p_{d} . l a b e l\right) \cdot j$ is a prefix of $v^{2} l_{i}$ (since $p_{i}=p_{d}$ ). Initially, the children of newNode $e_{i}$ are the new leaf node whose label is val $_{i}$ and nodeSibling . $_{\text {. }}$ Since $\left(g p_{d} . l a b e l\right) \cdot j$ is a prefix of nodeSibling llabel $_{\text {and }}$ val $_{i}$, the $k$ th bit of new Node $e_{i} . l a b e l$ is $j$.

Case 6: $I$ is created at line 70 Let $\left\langle g p_{d}, p_{d}\right.$, node $\left.e_{d},-,-,-\right\rangle$ be the result returned by the call to $\operatorname{search}\left(v a l_{d}\right)$ on line 45 that precedes the creation of $I$ and $\left\langle-, p_{i}\right.$, node $\left._{i},-,-,-\right\rangle$ be the result returned by the call to $\operatorname{search}\left(v_{a} l_{i}\right)$ on line 47 that precedes the creation of $I$. Then, newChild $d_{i}$ is the new internal node that is created at line 67 and newNode is the new internal node that is created at line 69 Then, node $_{i}=g p_{d}, I . p N o d e[0]=p_{i}$, I.oldChild $[0]=$ node $_{i}$ and I.newChild $[0]=$ newNode ${ }_{i}$. Let nodeSibling ${ }_{d}$ be the child of $p_{d}$ that is read at line 50 and $p$ Sibling $g_{d}$ be a child of $g p_{d}$ that is read at line 66. By Lemma 6, $p_{i} \cdot \operatorname{child}[j]=\operatorname{node}_{i}=g p_{d}$ for some $j$ at some time during $\operatorname{search}\left(\operatorname{val}_{i}\right)$. By Invariant 7. $\left(p_{i} . l a b e l\right) \cdot j$ is a prefix of $g p_{d} . l a b e l, g p_{d} . l a b e l$ is a prefix of $p$ Sibling $g_{d} . l a b e l$ and of $p_{d} . l a b e l$ and

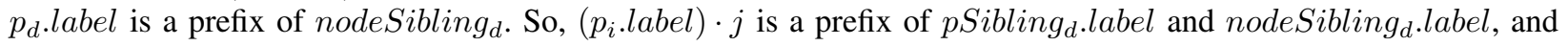
the $k$ th bit of node $e_{i}$ label is $j$.

Initially, the children of newChild $d_{i}$ are SSibling $_{d}$ and nodeSibling . Since $\left(p_{i}\right.$. label $) \cdot j$ is a prefix of

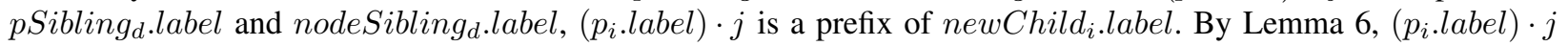
is a prefix of $v l_{i}$. Initially, the children of newNode $i$ are the new leaf node whose label is val $_{i}$ and newChild . $_{\text {. }}$ Since $\left(p_{i} . l a b e l\right) \cdot j$ is a prefix of newChild ${ }_{i} . l a b e l$ and $v a l_{i}$, the $k$ th bit of newNode ${ }_{i}$.label is $j$.

In the implementation, update operations might help one another to change the child fields of nodes by calling the help routine. So, there might be several CAS steps that try to change an element of the child field of some internal node from a value old to some value new. The following lemmas show that, as long as there is no ABA problem on the child field of an internal node, the first child CAS of $I$ on I.pNode $[i]$ succeeds and no subsequent ones succeed.

Later we shall use these facts to prove inductively that there is no ABA problem on child fields.

Lemma 30. Assume that before some time $T$, for all internal nodes $y$, no child CAS sets y.child $[k]$ to a value old after a child CAS of the form CAS(y.child[k], old, -) succeeds.

Let I be a Flag object. Then, for each $i$, only the first child CAS of I on I.pNode[i] can succeed at $T$ or before $T$.

Proof: Let $x=I . p N o d e[i]$ and old $=I$.oldChild $[i]$. Then, the child CAS of $I$ on I.pNode $[i]$ is of the form CAS (x.child $[j]$, old, -) for some $j$. Let $T_{1 c}$ be the time when the first child CAS of $I$ on I.pNode $[i]$ occurs. Suppose some other child CAS of $I$ on $I . p N o d e[i]$ occurs at $T_{c}$ where $T_{1 c}<T_{c} \leq T$. We shall show the child CAS at $T_{c}$ fails.

By Observation 27, x.child $[j]$ was old at some time before $I$ was created (before $T_{1 c}$ ). If $x$. child $[j]=$ old immediately before $T_{1 c}$, then the child CAS at $T_{1 c}$ changes $x$.child $[j]$ from old to a different value (by Lemma 28). Thus, just after $T_{1 c}, x . c h i l d[j] \neq$ old. So, at $T_{1 c}$ or some time before $T_{1 c}$, a child CAS of the form CAS $(x . c h i l d[j]$, old, -) succeeds. By the assumption, x.child $[j]$ is not changed back to old between $T_{1 c}$ and $T$, so the child CAS at time $T_{c}$ fails.

Let $I$ be a Flag object. The following lemma shows that if the first child CAS of $I$ on I.pNode $[i]$ for some $i$ occurs, no other child CAS changes the child fields of nodes in $F_{I}$ between the time when the info fields of the nodes in $F_{I}$ are read for the last time before $I$ is created and the first child CAS of $I$ on I.pNode[i].

Lemma 31. Assume that before some time $T$, for all internal nodes $y$, no child $C A S$ sets $y . c h i l d[k]$ to a value old after a child CAS of the form CAS(y.child[k], old, -) succeeds.

Let I be a Flag object, $x=I$.flag $[i]$ and $x$ Info $=I$.oldInfo $[i]$ for some $i$. Suppose $x$.info $=x$ Info at time $T_{1}$ and for some $j$, the first child CAS of I on I.pNode[j] occurs at time $T_{2}>T_{1}$ and $T_{2}<T$. Then, no child CAS of any other Flag object $I^{\prime} \neq I$ changes x.child between $T_{1}$ and $T_{2}$.

Proof: By Lemma 30, for each $k$, only the first child CAS of Flag object $I^{\prime}$ on $I^{\prime}$.pNode $[k]$ can succeed. Thus, if a child CAS of $I^{\prime}$ changes x.child, by Lemma21 and 22, $x$.info $=I^{\prime}$ just before the CAS (since $x \in F_{I^{\prime}}$ by Observation 24. By Lemma 25, x.info is either $x \operatorname{Info}$ or $I$ at all times between $T_{1}$ and $T_{2}$. By Lemma 12 . 
$x$ Info is an Unflag object. Since $I \neq I^{\prime}, x$.info is never equal to $I^{\prime}$ between $T_{1}$ and $T_{2}$, so no child CAS of $I^{\prime}$ changes x.child between $T_{1}$ and $T_{2}$.

Lemma 32. Assume that before some time $T$, for all internal nodes $y$, no child $C A S$ sets y.child $[k]$ to a value old after a child CAS of the form CAS(y.child $[k]$, old, -) succeeds.

Let I be a Flag object. Then, for each $i$, if the first child CAS of I on I.pNode[i] occurs before T, then it succeeds.

Proof: Let $x=I . p N o d e[i]$, old $=$ I.oldChild $[i]$. Then, the child CAS of $I$ on I.pNode $[i]$ is of the form CAS (x.child $[j]$, old, -). Let $T_{1 c}$ be the time when the first child CAS of $I$ on I.pNode $[i]$ occurs (before $T$ ). We prove that it succeeds.

By Observation 24, $x=$ I.pNode $[i] \in F_{I}$. By Lemma 14 $x$.info $=$ I.oldInfo $[i]$ at time $T_{0}$ before $I$ is created. By Observation 27 and Lemma 6, x.child $[j]=$ old at some time between $T_{0}$ and $T_{1 c}$. So, to prove the lemma, it suffices to show that no child CAS changes $x$.child $[j]$ from old to another value between $T_{0}$ and $T_{1 c}$.

First, we argue that such a change cannot be made by a child CAS of $I$ itself. This can only happen if $I$ is created at line 55 or 57 and $i=1$ and the child CAS of $I$ at $T_{1 c}$ is on I.pNode[1]. For this case, we must show that no child CAS of $I$ on I.pNode $[0]$ changes $x$.child $[j]$ from old to another value: Since the condition at line $\mathrm{F}$ preceding the creation of $I$ is true, I.oldNode[0] $\neq$ I.oldNode[1]. If I.pNode[0] $=$ I.pNode[1], the child CAS of $I$ on I.pNode $[0]$ does not change $x . c h i l d[j]$ from old to another value (since I.oldNode $[0] \neq$ old).

By Lemma 31] no child CAS of $I^{\prime} \neq I$ changes $x$.child $[j]$ between $T_{0}$ and $T_{1 c}$.

Let $I$ be a Flag object and $x$ be an internal node in $F_{I}-U_{I}$. By Lemma 21 and 22, x. info $=I$ just before the first child CAS of $I$. If there is any child CAS of $I$, by Lemma 21, 22 and 23, x.info $=I$ at all times after the first child CAS of $I$. After the first child CAS of $I$, we say $x$ is marked by $I$. (Thus, once a node is marked by $I$, it remains marked by $I$ forever.)

Now, we prove if an internal node becomes unreachable, it is marked after that.

Lemma 33. Assume that before some time $T$, for all internal nodes $y$, no child $C A S$ sets y.child $[k]$ to a value old after a child CAS of the form CAS(y.child $[k]$, old, -) succeeds.

Let I be a Flag object and $x$ be an internal node. Consider a child CAS of I that succeeds at time $T^{\prime}$ (before $T)$. If $x$ is reachable immediately before $T^{\prime}$ and $x$ becomes unreachable at $T^{\prime}, x$ is marked by $I$ at all times after $T^{\prime}$.

Proof: Let $I$ be a Flag object. Let $\langle g p, p$, node,,,---$\rangle$ be the result returned by the call to $\operatorname{search}(v a l)$ on line 23,36 or 45 that precedes the creation of $I$. If $I$ is created inside replace $\left(v a l, v a l^{\prime}\right)$, let $\left\langle-, p^{\prime}\right.$, node $\left.^{\prime},-,-,-\right\rangle$ be the result returned by the call to $\operatorname{search}\left(v a l^{\prime}\right)$ on line 47 that precedes the creation of $I$. By Lemma 30, $T^{\prime}$ is the first child CAS of $I$ on I.pNode $[i]$ for some $i$. We consider different cases according to what line created $I$. For each case, we show that only nodes in $F_{I}-U_{I}$ could become unreachable immediately after $T^{\prime}$. By Lemma 21, 22 and 23, any node in $F_{I}-U_{I}$ is flagged by $I$ at all times after $T^{\prime}$.

Case 1: $I$ is created at line 30 Then, node is an internal node. Then, new Node is the new internal node created at line 27 whose non-empty children are a new leaf node and a new copy of node. In this case, I.oldChild $[0]=$ node and I.newChild $[0]=$ newNode. Since node $\in F_{I}$, by Lemma 31, node.child is not changed between the time when node.info is read at line 25 and $T^{\prime}$. Thus, just before $T^{\prime}$, the child field of the new copy of node is the same as node.child. The only internal node that could become unreachable at $T^{\prime}$ is node. In this case, node $\in F_{I}-U_{I}$. By the definition, node is marked at all times after $T^{\prime}$.

Case 2: $I$ is created at line 31. Then, node is a leaf node. In this case, I.oldChild $[0]=$ node is a leaf node, so no internal node becomes unreachable at $T^{\prime}$.

Case 3: $I$ is created at line 40 Let nodeSibling be the element of p.child that is read at line 38 . By Invariant 7 and Lemma 4, nodeSibling exists and is different from node. In this case, I.oldChild $[0]=p$ and I.newChild $[0]=$ nodeSibling. Since $p \in F_{I}$, by Lemma 31, p.child is not changed between the time when p.info is read for the last time during $\operatorname{search}(\mathrm{val})$ and $T^{\prime}$. Since $p$ is a parent of node during $\operatorname{search}(\mathrm{val})$ (by Lemma 6) and $p$ is a parent of nodeSibling at line 38, the children of $p$ just before $T^{\prime}$ are node and nodeSibling. Since the operation does not return false at line 37, node is a leaf node. So, the only internal node that could become unreachable at $T^{\prime}$ is $p$. In this case, $p \in F_{I}-U_{I}$. By the definition, node is marked at all times after $T^{\prime}$. 
Case 4: $I$ is created at line 55 or 57. Then, new Node $e_{i}$ is the new internal node that is created at line 53 If the first child CAS of $I$ on I.pNode [0] occurs at time $T^{\prime}$, an element of $p^{\prime}$.child is changed from node to newNode ${ }_{i}$ at $T^{\prime}$. By the same argument as in Case 1 and 2, if node $e^{\prime}$ is an internal node, node $e^{\prime}$ is the only internal node that could become unreachable at $T^{\prime}$ and node $e^{\prime}$ is marked at all times after $T^{\prime}$.

Let nodeSibling $g_{d}$ be an element of p.child that is read at line 50 If the first child CAS of $I$ on I.pNode[1] occurs at time $T^{\prime}$, an element of gp.child is changed from $p$ to nodeSibling $g_{d}$ at $T^{\prime}$. By the same argument as in Case 3, $p$ is the only internal node that could become unreachable at $T^{\prime}$ and $p$ is marked at all times after $T^{\prime}$.

Case 5: $I$ is created at line 59. Then, I.oldChild $[0]=$ node $^{\prime}$. Since the operation does not return false at line 46 node' is a leaf node. Since I.oldChild[0] is a leaf node, no internal node becomes unreachable at $T^{\prime}$.

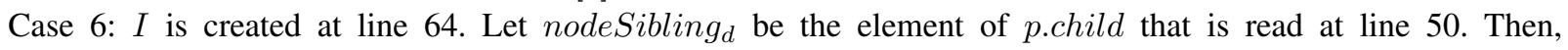
newNode ${ }_{i}$ is the new internal node created at line 62 whose children are a new leaf node and nodeSibling . $_{\text {. }}$

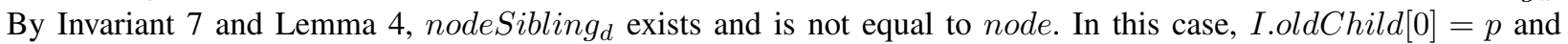
I.newChild $[0]=$ newNode ${ }_{i}$. By Lemma 31, since $p \in F_{I}$, p.child is not changed between the time when $p$.info is read for the last time during $\operatorname{search}(\mathrm{val})$ and $T^{\prime}$. Since node $\neq$ nodeSibling, by Lemma 4 , the children of $p$ are node and nodeSibling just before $T^{\prime}$. Since the operation does not return false at line 46 node is a leaf node. So, the only internal node that could become unreachable at $T^{\prime}$ is $p$. In this case, $p \in F_{I}-U_{I}$. By the definition, $p$ is marked at all times after $T^{\prime}$.

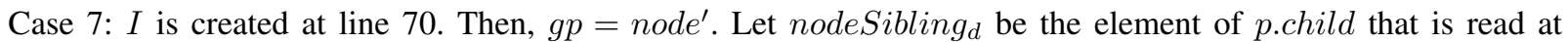
line 50 and Sibling $_{d}$ be the element of gp.child that is read at line 66 Then, newChild $d_{i}$ is the new internal node created at line 67 whose children are nodeSibling ${ }_{d}$ and pSibling $_{d}$ and newNode ${ }_{i}$ is the new internal node created at line 69 whose children are a new leaf node and newChild ${ }_{i}$. In this case, I.oldChild $[0]=g p$ and I.newChild $[0]=$ new Node ${ }_{i}$. In this case, gp $\in F_{I}$ and $p \in F_{I}$. By Lemma 31, gp.child is not changed between the time when gp.info is read for the last time during $\operatorname{search}(\mathrm{val})$ and $T^{\prime}$. By Lemma 31, p.child is not changed between the time when p.info is read for the last time during $\operatorname{search}(v a l)$ and $T^{\prime}$. So, just before $T^{\prime}$, the children

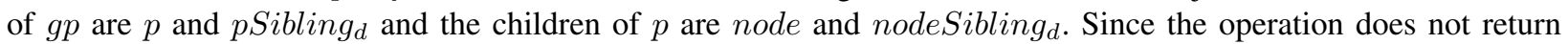
false at line 46, node is a leaf node. So, the only internal nodes that become unreachable at $T^{\prime}$ are $g p=n o d e^{\prime}$ and $p$. In this case, $g p=$ node $e^{\prime}$ and $p$ are in $F_{I}-U_{I}$. By the definition, $p$ and $g p$ are marked at all times after $T^{\prime}$.

The following corollary shows if an internal node is not marked, it has not become unreachable.

Corollary 34. Assume that before some time $T$, for all internal nodes $y$, no child CAS sets y.child $[k]$ to a value old after a child CAS of the form CAS(y.child[k], old, -) succeeds.

If an internal node $x$ is not marked at time $T^{\prime}$ (before $T$ ) and $x$ was reachable at some time before $T^{\prime}$, then $x$ is reachable at time $T^{\prime}$.

Now, we show that, for each $i$, I.oldChild $[i]$ becomes unreachable just after the successful child CAS of $I$ on I.pNode $[i]$.

Lemma 35. Assume that before some time $T$, for all internal nodes $y$, no child $C A S$ sets y.child $[k]$ to a value old after a child CAS of the form CAS(y.child $[k]$, old, -) succeeds.

Let I be a Flag object. If the child CAS of I on I.pNode[i] succeeds at time $T^{\prime}$ (before $T$ ) for some $i$, I.oldChild[i] becomes unreachable just after $T^{\prime}$.

Proof: Let $I$ be a Flag object. Let $\langle g p, p$, node, -, -, - $\rangle$ be the result returned by the call to $\operatorname{search}(v a l)$ on line 23,36 or 45 that precedes the creation of $I$. If $I$ is created inside replace $\left(v_{a l}, v_{a l}^{\prime}\right)$, let $\left\langle-, p^{\prime}\right.$, node $\left.^{\prime},-,-,-\right\rangle$ be the result returned by the call to $\operatorname{search}\left(v a l^{\prime}\right)$ on line 47 that precedes the creation of $I$. By Lemma 30, $T^{\prime}$ is the first child CAS of $I$ on I.pNode $[i]$. We consider different cases according to what line created $I$.

Case 1: $I$ is created at line 30 or 31 . Let newNode be the new internal node created at line 27 whose non-empty children are a new leaf node and a new copy of node. By Invariant 7. node is not descendant of the new copy of node and hence is not a descendant of newNode. In this case, I.oldChild $[0]=$ node and I.newChild $[0]=$ newNode. Since $p \in F_{I}, p$ is a parent of node just before $T^{\prime}$ (by Lemma 31. Since $p$.info $=I$ just before $T^{\prime}$ (by Lemma 21 and 22, $p$ is not marked just before $T^{\prime}$ and $p$ is reachable just before $T^{\prime}$ (by Corollary 34). By Lemma $9 p$ is the only reachable parent of node just before $T^{\prime}$. Since node is not a descendant of newNode, node becomes unreachable just after $T^{\prime}$. 
Case 2: $I$ is created at line 40. Let nodeSibling be the element of p.child that is read at line 38 . By Invariant 7 and Lemma 4, nodeSibling exists and is different from node. In this case, I.oldChild $[0]=p$ and I.newChild $[0]=$ nodeSibling. Since $p$ and $g p$ are in $F_{I}, g p$ is a parent of $p$ and $p$ is a parent of node just before $T^{\prime}$ (by Lemma 31). Since gp.info $=I$ just before $T^{\prime}$ (by Lemma 21 and 22), $g p$ is not marked just before $T^{\prime}$ and $g p$ is reachable just before $T^{\prime}$ (by Corollary 34. Since $g p$ is the reachable parent of $p$ just before $T^{\prime}, p$ is reachable just before $T^{\prime}$. By Lemma $9, p$ is the only reachable parent of node just before $T^{\prime}$. Since node is not a descendant of nodeSibling (by Invariant 7), node becomes unreachable just after $T^{\prime}$.

Case 3: $I$ is created at line 55 or 57. Let newNode $e_{i}$ be the new internal node that is created at line 53 If the first child CAS of $I$ on I.pNode[0] occurs at time $T^{\prime}$, an element of $p^{\prime}$.child is changed from node to new Node $e_{i}$ at $T^{\prime}$. By the same argument as in Case 1, node $e^{\prime}$ becomes unreachable just after $T^{\prime}$.

Let nodeSibling $g_{d}$ be an element of p.child that is read at line 50 If the first child CAS of $I$ on I.pNode[1] occurs at time $T^{\prime}$, an element of gp.child is changed from $p$ to nodeSibling at $^{\prime}$. By the same argument as in Case 2, node becomes unreachable just after $T^{\prime}$.

Case 4: $I$ is created at line 59. Then, node $=$ node $e^{\prime}$ I.oldChild $[0]=$ node $^{\prime}$ and I.newChild $[0]$ is a new leaf node. Since $p \in F_{I}, p$ is a parent of node $=$ node ${ }^{\prime}$ just before $T^{\prime}$ (by Lemma 6 and 31. Since $p . i n f o=I$ just before $T^{\prime}$ (by Lemma 21 and 22), $p$ is reachable just before $T^{\prime}$ (by Corollary 34). Since I.newChild[0] is a leaf node and $p$ is the only reachable parent of node $=$ node just before $T^{\prime}$ (by Lemma 9 , node $e^{\prime}$ becomes unreachable just after $T^{\prime}$.

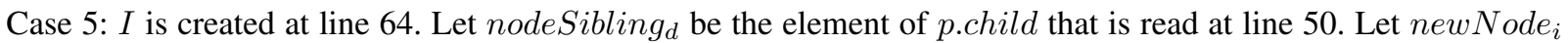
be the new internal node created at line 62 whose children are a new leaf node and nodeSibling . By Invariant 7

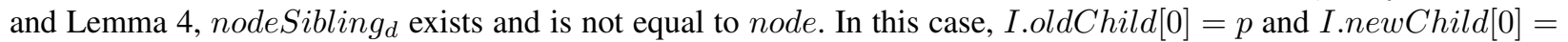

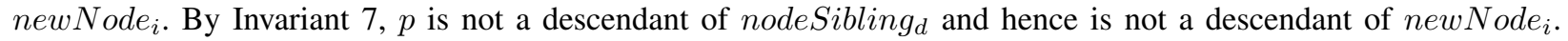
Since $g p \in F_{I}, g p$ is a parent of $p$ just before $T^{\prime}$ (by Lemma 31). Since gp.info $=I$ just before $T^{\prime}$ (by Lemma 21 and 22), $g p$ is not marked just before $T^{\prime}$ and $g p$ is reachable just before $T^{\prime}$ (by Corollary 34). By Lemma 9 , gp is the only reachable parent of $p$ just before $T^{\prime}$. Since $p$ is not a descendant of new Node $e_{i}, p$ becomes unreachable just after $T^{\prime}$.

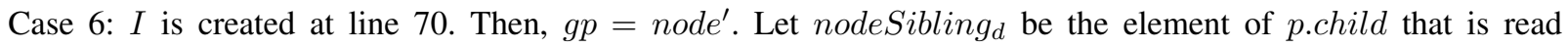
at line 50 and $p$ Sibling $_{d}$ be the element of gp.child that is read at line 66 . Let newChild ${ }_{i}$ be the new internal node created at line 67 whose children are nodeSibling and $_{\text {SSibling }}$. Let newNode $e_{i}$ be the new internal node created at line 69 whose children are a new leaf node and newChild ${ }_{i}$. In this case, I.oldChild $[0]=g p$ and I.newChild $[0]=$ new Node $_{i}$. By Invariant 7 gp is not a descendant of nodeSibling ${ }_{d}$, pSibling $_{d}$, newChild ner new Node ${ }_{i}$. Since $p^{\prime} \in F_{I}, p^{\prime}$ is a parent of node $e^{\prime}=g p$ just before $T^{\prime}$ (by Lemma 31). Since $p^{\prime}$.info $=I$ just before $T^{\prime}$ (by Lemma 21 and 22, $p^{\prime}$ is not marked just before $T^{\prime}$ and $p^{\prime}$ is reachable just before $T^{\prime}$ (by Corollary 34. By Lemma 9, $p^{\prime}$ is the only reachable parent of node $=g p$ just before $T^{\prime}$. Since $g p$ is not a descendant of new ode $_{i}$, gp becomes unreachable just after $T^{\prime}$.

We say that the search operation visits the node that gets stored in node when the search performs line 78 or 82 The following lemma shows that a search operation does not visit a node that was unreachable at all times during the operation.

Lemma 36. Assume that before some time $T$, for all internal nodes $y$, no child $C A S$ sets y.child $[k]$ to a value old after a child CAS of the form CAS(y.child $[k]$, old, -) succeeds.

Assume a search operation visits a node $x$ Child $\neq$ root by reading $x$ Child in the child field of some node $p N o d e$ before T. Then, there is a time after the search operation begins and before the search operation visits $x$ Child that $p N o d e$ is reachable and a parent of $x$ Child.

Proof: We prove the lemma by induction on the number of steps that the search operation has done. Let $T^{\prime}$ be a time that the search operation visits a node $x$ Child (before $T$ ). We assume the lemma is true for all nodes visited before $T^{\prime}$. Now, we show that the lemma is true for $x$ Child. Then, $x$ Child is visited on line 82 at time $T$. First, we show that $p N o d e$ is visited earlier during the operation.

If $x$ Child is visited at line 82 , $p N o d e$ is visited earlier at line 78 or at line 82 during the previous loop iteration. Thus, $p N o d e$ is visited at some time during the search operation before time $T^{\prime}$. So, $p N o d e$ was reachable at some time $T^{\prime \prime}$ after the search operation begins and before the search operation visited pNode. If $x$ Child was a child of $p N o d e$ at $T^{\prime \prime}$, the lemma is proved. 
Otherwise, an element of $p N o d e . c h i l d$ is set to $x$ Child at some later time between $T^{\prime \prime}$ and $T^{\prime}$. Thus, a child CAS sets an element of $p N$ ode.child to $x$ Child between $T^{\prime \prime}$ and $T^{\prime}$. Let $I$ be a Flag object such that a child CAS of $I$ sets an element of $p N o d e . c h i l d$ to $x$ Child between $T^{\prime \prime}$ and $T^{\prime}$. By Lemma 32, the first child CAS of $I$ on $p N o d e$ succeeds between $T^{\prime \prime}$ and $T^{\prime}$. By Observation 24, $p N o d e \in F_{I}$. By Corollary 21 and 22, pNode.info $=I$ just before the child CAS of $I$ between $T^{\prime \prime}$ and $T^{\prime}$. By Observation 24 pNode $\in U_{I}$. So, pNode is not marked just before the child CAS of $I$. Since $p N o d e$ was reachable at $T^{\prime \prime}$, by Corollary 34 pNode is still reachable just before the child CAS of $I$. So, pNode is reachable and a parent of $x$ Child just after the child CAS of $I$ between $T^{\prime \prime}$ and $T^{\prime}$.

Now, we show, after a node becomes unreachable, the node does not become reachable again.

Lemma 37. Assume that before some time $T$, for all internal nodes $y$, no child CAS sets y.child $[k]$ to a value old after a child CAS of the form CAS(y.child[k], old, -) succeeds.

Let $x$ be a Node object. If, after $x$ is reachable, $x$ becomes unreachable at time $T^{\prime}$ (before $T$ ), $x$ does not become reachable again between $T^{\prime}$ and $T$.

Proof: To derive a contradiction, assume the lemma is false. Let $T^{\prime \prime}$ (before $T$ ) be the first time it is violated and $x$ be the highest node in the tree at $T^{\prime \prime}$ that violates the lemma. Let $T^{\prime}$ be the time that $x$ becomes unreachable before $T^{\prime \prime}$. So, $x$ becomes reachable when a child CAS is performed at $T^{\prime \prime}$. We have two different cases.

Case 1: a child CAS of some Flag object $I$ sets an element of a child field to $x$ at $T^{\prime \prime}$. If $I$ and $x$ are created in the same loop iteration of an update operation, $x$ is not reachable at any time before $T^{\prime \prime}$. So, $I$ is created at line 40, 55 or 57. Let $\left\langle g p_{d}, p_{d},-,-,-,-\right\rangle$ be the result returned by the call to the search operation on line 36 or 45 that precedes the creation of $I$. If $I$ is created at line 40. I.newChild $[0]=x$ and if $I$ is created at line 55 or 57 . I.newChild $[1]=x$. Then, $x$ is read as a child of $p_{d}$ at line 38 or 50 and an element of $g p_{d}$.child is set to $x$ at $T^{\prime \prime}$. By Observation 24, $g p_{d} \in F_{I}$ and $g p_{d} \in U_{I}$.

By Lemma 32, the first child CAS of $I$ on $g p_{d}$ succeeds at $T^{\prime \prime}$. By Lemma 21 and 22, $g p_{d}$. info $=I$ just before $T^{\prime \prime}$. Since $g p_{d}$ is not marked just before $T^{\prime \prime}$ and $g p_{d}$ was reachable during the search operation by Lemma 36, $g p_{d}$ is reachable just before $T^{\prime \prime}$ by Corollary 34 . Since $p_{d}$ was a child of $g p_{d}$ during the search operation (by Lemma 6), $p_{d}$ is a child of $g p_{d}$ just before $T^{\prime \prime}$ (by Lemma 31). Since $g p_{d}$ is reachable just before $T^{\prime \prime}, p_{d}$ is reachable just before $T^{\prime \prime}$. By Lemma 31, since $p_{d} \in F_{I}$, p.child is not changed between the time when p.info was read for the last time during the search operation and $T^{\prime \prime}$. Since $x$ was a child of $p_{d}$ at line 38 or 50, $x$ is still a child of $p_{d}$ just before $T^{\prime \prime}$. Since $p_{d}$ is reachable just before $T^{\prime \prime}, x$ is reachable just before $T^{\prime \prime}$, contradicting the fact that $x$ becomes reachable at $T^{\prime \prime}$ for the first time after $T^{\prime}$.

Case 2: the child CAS of some Flag object $I$ sets an element of a child field to a proper ancestor anc of $x$ at $T^{\prime \prime}$. Since $x$ is not reachable just before $T^{\prime \prime}$, anc is also not reachable just before $T^{\prime \prime}$. Since $x$ is the highest node that violates the lemma at $T^{\prime \prime}$, anc is not reachable at any time before $T^{\prime \prime}$. So, anc is created at line 121 inside createNode that is called at line 27, 53, 62 or 69 prior to the creation of $I$. We consider different cases depending on what line calls createNode that created anc.

Case 2A: anc is created at line 121, which is called from line 27 or 53 . Let $\left\langle-, p_{i}\right.$, node $\left._{i},-,-,-\right\rangle$ be the result returned by the call to the search operation on line 23 or 47 that precedes the creation of $I$. The children of anc initially are a new copy of node $e_{i}$ that is created at line 26 or 52 and the leaf node that is created at line 27 or 53 Since $x$ was reachable before $T^{\prime}, x$ is not created at line 26, 27, 52 or 53 that precedes the creation of $I$. So, $x$ is not a child of anc just before $T^{\prime \prime}$. Since anc is an ancestor of $x$ just before $T^{\prime \prime}$, a child of anc is an internal node just before $T^{\prime \prime}$. So, node $_{i}$ is an internal node and $x$ is a descendant of a new copy of node $e_{i}$ just before $T^{\prime \prime}$. So,

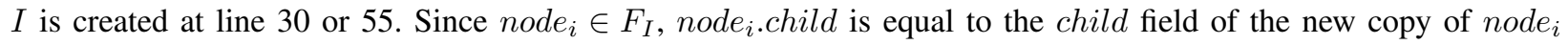
just before $T^{\prime \prime}$ (by Lemma 31). So, $x$ is also a proper descendant of node ${ }_{i}$ just before $T^{\prime \prime}$. Let nodeChild be a child of node $_{i}$ at line 26 or 52 such that $x$ is a descendant of nodeChild just before $T^{\prime \prime}$.

By Observation 24, $p_{i} \in F_{I}$. Since $p_{i}$. info $=I$ just before $T^{\prime \prime}$ (by Lemma 21 and 22, $p_{i}$ is not marked just before $T^{\prime \prime}$. Since $p_{i}$ was reachable during the search operation (by Lemma 36, $p_{i}$ is reachable just before $T^{\prime \prime}$ by Corollary 34 By Lemma $6 p_{i}$ is a parent of node $e_{i}$ at some time during the search operation before $T^{\prime \prime}$. Since $p_{i}$ is a parent of node $i_{i}$ just before $T^{\prime \prime}$ (by Lemma 31p and $p_{i}$ is reachable just before $T^{\prime \prime}$, node ${ }_{i}$ is reachable just before $T^{\prime \prime}$. Since node ${ }_{i}$.child is not changed between line 26 or 52 and $T^{\prime \prime}$ (by Lemma 31, nodeChild is a child of node $_{i}$ just before $T^{\prime \prime}$. Since node $_{i}$ is reachable just before $T^{\prime \prime}$, nodeChild is reachable just before $T^{\prime \prime}$. Since $x$ is 
a descendant of nodeChild just before $T^{\prime \prime}, x$ is reachable just before $T^{\prime \prime}$, contradicting that $x$ becomes reachable at $T^{\prime \prime}$ for the first time after $T^{\prime}$.

Case 2B: anc is created at line 121, which is called from line 62. Then, $I$ is created at line 64. Let $\left\langle g p_{d}, p_{d}\right.$, node $\left._{d},-,-,-\right\rangle$ be the result returned by the call to search $\left(\right.$ val $\left._{d}\right)$ on line 45 that precedes the creation of $I$. Let nodeSibling $g_{d}$ be the node that is read from $p_{d}$.child at line 50. The children of anc initially are nodeSibling $g_{d}$ and the leaf node that is created at line 62. Since $x$ was reachable before $T^{\prime}, x$ is not created at line 62 that precedes the creation of $I$. Since anc is an ancestor of $x$ just before $T^{\prime \prime}, x$ is a descendant of nodeSibling $d_{d}$ just before $T^{\prime \prime}$.

In this case, $g p_{d} \in F_{I}$ and $p_{d} \in F_{I}$. By Observation 24 $g p_{d} \in U_{I}$. Since $g p_{d}$.info $=I$ just before $T^{\prime \prime}$ (by Lemma 21 and 22, $g p_{d}$ is not marked just before $T^{\prime \prime}$. Since $g p_{d}$ was reachable during the search operation (by Lemma 36, $g p_{d}$ is reachable just before $T^{\prime \prime}$ (by Corollary 34). By Lemma 6, $g p_{d}$ is a parent of $p_{d}$ at some time during the search operation. So, $g p_{d}$ is a parent of $p_{d}$ just before $T^{\prime \prime}$ (by Lemma 31). Since $g p_{d}$ is reachable just before $T^{\prime \prime}, p_{d}$ is reachable just before $T^{\prime \prime}$. By Lemma 31, nodeSibling $g_{d}$ is a child of $p_{d}$ just before $T^{\prime \prime}$. Since $p_{d}$ is reachable just before $T^{\prime \prime}$, nodeSibling $g_{d}$ is reachable just before $T^{\prime \prime}$. Since $x$ is a descendant of nodeSibling $g_{d}$ just before $T^{\prime \prime}, x$ is reachable just before $T^{\prime \prime}$, contradicting that $x$ becomes reachable at $T^{\prime \prime}$ for the first time after $T^{\prime}$.

Case 2C: anc is created at line 69 Then, $I$ is created at line 70 . Let $\left\langle g p_{d}, p_{d}\right.$, node $\left.e_{d},-,-,-\right\rangle$ be the result returned by the call to search $\left(v a l_{d}\right)$ on line 45 that precedes the creation of $I$ and $\left\langle-, p_{i}\right.$, node $\left._{i},-,-,-\right\rangle$ be the result returned by the call to $\operatorname{search}\left(v a l_{i}\right)$ on line 47 that precedes the creation of $I$. Let nodeSibling $g_{d}$ be the child of $p_{d}$ that is read at line 50 and SSibling $_{d}$ be the child of $g p_{d}$ that is read at line 66. Let newChild $d_{i}$ be the internal node that is created at line 67. Initially, the children of newChild $d_{i}$ are nodeSibling $g_{d}$ and pSibling $_{d}$. The children of anc initially are newChild $d_{i}$ and the leaf node that is created at line 69. Since $x$ was reachable before $T^{\prime}, x$ is not created at line 67 or 69 that precedes the creation of $I$. Since anc is an ancestor of $x$ just before $T^{\prime \prime}$ and $x$ is not created at line 67 or 69, $x$ is a descendant of nodeSibling or of $p$ Sibling $g_{d}$ just before $T^{\prime \prime}$.

In this case, $n o d e_{i}=g p_{d}$. Since $I$ is created at line 70, by the pseudo-code, $F_{I}=\left\{p_{i}, g p_{d}, p_{d}\right\}$. Since $p_{i}$.info $=I$ just before $T^{\prime \prime}$ (by Lemma 21 and 22, $p_{i}$ is not marked just before $T^{\prime \prime}$. Since $p_{i}$ was reachable during the search operation (by Lemma 36, $p_{i}$ is reachable just before $T^{\prime \prime}$ by Corollary 34. By Lemma 6 and 31, $p_{i}$ is a parent of node $_{i}=g p_{d}$ just before $T^{\prime \prime}$ and $g p_{d}$ is a parent of $p_{d}$ just before $T^{\prime \prime}$. Since $p_{i}$ is reachable just before $T^{\prime \prime}, g p_{d}$ and $p_{d}$ are reachable just before $T^{\prime \prime}$. By Lemma 31, nodeSibling $g_{d}$ is a child of $p_{d}$ just before $T^{\prime \prime}$ and $p$ Sibling is a child of $g p_{d}$ just before $T^{\prime \prime}$. Since $p_{d}$ and $g p_{d}$ are reachable just before $T^{\prime \prime}$, nodeSibling $g_{d}$ and $p$ Sibling $g_{d}$ are reachable just before $T^{\prime \prime}$. Since $x$ is a descendant of nodeSibling $g_{d}$ or of $p$ Sibling $_{d}$ just before $T^{\prime \prime}, x$ is reachable just before $T^{\prime \prime}$, contradicting that $x$ becomes reachable at $T^{\prime \prime}$ for the first time after $T^{\prime}$.

Now, we show that the ABA problem on the child field of internal nodes is avoided.

Lemma 38. Let $x$ and $y$ be internal nodes. After a child CAS of the form CAS(x.child[i], old, -) succeeds, no child CAS sets y.child $[j]$ to old.

Proof: To derive a contradiction, assume the lemma is violated for the first time at $T$. Let $I$ be a Flag object such that the first child CAS of $I$ of the form CAS(x.child $[i]$, -, old) succeeds at $T$ and let $I^{\prime}$ be a Flag object such that the first child CAS of $I^{\prime}$ of the form CAS(y.child $[j]$, old, -) succeeds at some earlier time $T^{\prime}$.

Since the child CAS of $I^{\prime}$ changes y.child $[j]$ from old to other value at $T^{\prime}, y . \operatorname{child}[j]=$ old just before $T^{\prime}$. By Observation 24, $y \in F_{I}$. Since $y$.info $=I^{\prime}$ just before $T^{\prime}$ (by Lemma 21 and 22), $y$ is not marked just before $T^{\prime}$. By Lemma 36, $y$ was reachable at some earlier time. So, $y$ is reachable just before $T^{\prime}$ (by Corollary 34). Since old is a child $y$ just before $T^{\prime}$, old is reachable just before $T^{\prime}$.

If old is created at line 27, 53, 59, 62 or 69 that precedes the creation of $I$, old could not become reachable before the child CAS of $I$ at $T$. So, old and $I$ are not created in the same loop iteration of an update operation. Thus, $I$ is created at line 40,55 or 57 . Let $\left\langle g p_{d}, p_{d}\right.$, node $\left.e_{d},-,-,-\right\rangle$ be the result returned by the call to the search operation at line 36 or 45 that precedes the creation of $I$. Then, $x=g p_{d}$ and old is read as a child of $p_{d}$ at line 38 or 50 Since $g p_{d} \in F_{I}, g p_{d}$.info $=I$ just before $T$ (by Lemma21 and 22. So, $g p_{d}$ is not marked just before $T$. By Lemma 6, $g p_{d}$ was a parent of $p_{d}$ at some time during the search operation. By Lemma 31, $g p_{d}$ was a parent of $p_{d}$ just before $T$. Since $g p_{d}$ is reachable just before $T, p_{d}$ is reachable just before $T$. By Lemma 31 , old is a child of $p_{d}$ just before $T$. Since $p_{d}$ is reachable just before $T$, old is reachable just before $T$. By Lemma 35, old becomes unreachable just after $T^{\prime}$ (before $T$ ), contradicting Lemma 37 . 
Since there is no ABA problem on the child field of nodes, Corollary 34 and Lemma 30, 31, 32, 33, 35, 36 and 37. which we proved earlier with the assumption that there is no ABA on the child fields of nodes is true without the assumption.

By Lemma 21, 22, 32 and 38, we have the following corollary.

Corollary 39. Let I be a Flag object and $x \in F_{I}$. Then, x.info $=I$ when a successful child CAS of I occurs.

\section{Correctness of the Search Operation}

In this section, we first show the post-conditions of the search operation are satisfied. Then, we show how to linearize search operations that terminate.

Let $I$ be a Flag object that is created at line 55 or 57 Then, the number of elements of I.pNode, I.oldChild and I.newChild is two and, by Observation 26, I.rmvLeaf is set to a non-empty leaf node. By Observation 26. I.rmvLeaf is flagged at line 95 before the first child CAS of $I$ at line 98 Any time after the first child CAS of $I$, we say I.rmvLeaf is logically removed. The following lemma shows that after a leaf node is flagged by a Flag object, the leaf node is not flagged by another Flag object.

Lemma 40. Let I be a Flag object that is created at line 55 or 57 If I.rmvLeaf.info is set to I by line 95 at time T, I.rmvLeaf.info is not set to another Flag object $I^{\prime} \neq I$ before $T$.

Proof: Let $\left\langle g p_{d}, p_{d}, n_{0} e_{d},-,-, r m v d_{d}\right\rangle$ be the result returned by the call to search $\left(v a l_{d}\right)$ on line 45 that precedes the creation of $I$. Then, I.rmvLeaf $=$ node $_{d}$. First, we show the following claim.

Claim: Let $I^{\prime \prime}$ be a Flag object that is created at line 55 or 57 and $\left\langle g p_{d}^{\prime \prime}, p_{d}^{\prime \prime},-,-,-,-\right\rangle$ be the result returned by the call to $\operatorname{search}\left(v a l_{d}^{\prime \prime}\right)$ on line 45 that precedes the creation of $I^{\prime \prime}$.

If all nodes in $F_{I}^{\prime \prime}$ get flagged successfully, $p_{d}^{\prime \prime}$ is reachable at all times between the time when $p_{d}^{\prime \prime}$ was reachable during $\operatorname{search}\left(v_{a l}^{\prime \prime}\right)$ and the first child CAS of $I^{\prime \prime}$.

Proof of Claim. Since $I^{\prime \prime}$ is created at line 55 or $57, g p_{d}^{\prime \prime} \in F_{I^{\prime \prime}}$. By Lemma 36, $g p_{d}^{\prime \prime}$ is reachable at some time during $\operatorname{search}\left(v a l_{d}^{\prime \prime}\right)$. Since $g p_{d}^{\prime \prime}$.info $=I^{\prime \prime}$ just before the first child CAS of $I^{\prime \prime}$ (by Corollary 39p, $g p_{d}^{\prime \prime}$ is not marked just before the first child CAS of $I^{\prime \prime}$. So, $g p_{d}^{\prime \prime}$ is reachable just before the first child CAS of $I^{\prime \prime}$ (by Corollary 34). By Lemma 6 $g p_{d}^{\prime \prime}$ was a parent of $p_{d}^{\prime \prime}$ at some time during the search operation. By Lemma 31, $g p_{d}^{\prime \prime}$ is a parent of $p_{d}^{\prime \prime}$ just before the first child CAS of $I^{\prime \prime}$. Since $g p_{d}^{\prime \prime}$ is reachable just before the fist child CAS of $I^{\prime \prime}, p_{d}^{\prime \prime}$ is reachable just before the first child CAS of $I^{\prime \prime}$. By Lemma 37. $p_{d}^{\prime \prime}$ is reachable at all times between the time when $p_{d}^{\prime \prime}$ was reachable during $\operatorname{search}\left(v_{a l} l_{d}^{\prime \prime}\right)$ and the first child CAS of $I^{\prime \prime}$. This completes the proof of the claim.

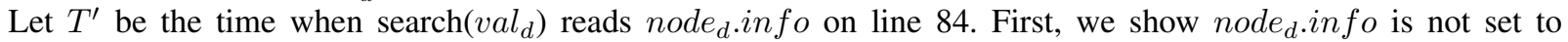
a Flag object at any time before $T^{\prime}$. Then, we show node .info is not set to a Flag object $I^{\prime} \neq I$ at any time between $T^{\prime}$ and $T$.

If node .info is an Unflag object at $T^{\prime}$, by Observation 26, node .info is not set to a Flag object at any time before $T^{\prime}$. We show that node .info is an Unflag object at time $T^{\prime}$. We prove it by contradiction. Assume node $_{d}$ info is some Flag $I^{\prime}$ at $T^{\prime}$. Let $\left\langle-, p_{d}^{\prime}\right.$, node $\left.e_{d},-,-,-\right\rangle$ be the result returned by the call to $\operatorname{search}\left(\right.$ val $\left._{d}^{\prime}\right)$ on line 45 that precedes the creation of $I^{\prime}$. Since the replace operation that creates $I$ does not return false at the execution of line 46 that precedes the creation of $I, r m v d_{d}$ is false. Since rmvd $_{d}=$ false and node .info $=I^{\prime}$ at line $84, I^{\prime} . p N o d e[0]$ was a parent of $I^{\prime}$.oldChild $[0]$ at line 124 during $\operatorname{search}\left(v_{a l} l_{d}\right)$. By Lemma 32 and 38 , no child CAS of $I^{\prime}$ on $I^{\prime}$.pNode[0] occurs before $T^{\prime}$. By Observation 26, all nodes in $F_{I^{\prime}}$ are flagged by $I^{\prime}$ before $T^{\prime}$ by Lemma 22

By the claim, $p_{d}^{\prime}$ is reachable just before $T^{\prime}$. Since $p_{d}$.info is set to $I$ after $\operatorname{search}\left(\mathrm{val}_{d}\right)$ and before the first child CAS of $I$ by Corollaryflag-node-before-child-cas-col. So, $p_{d}$.info is read for the last time during $\operatorname{search}\left(v_{a l} l_{d}\right)$ before $T^{\prime}$ and $p_{d}$.info is changed from that value to $I$ after $T^{\prime}$. By Lemma 15 and 12 , $p_{d}$.info is an Unflag object at $T^{\prime}$. Since $p_{d}^{\prime}$.info $=I^{\prime}$ at $T^{\prime}$ and $p_{d}$.info is an Unflag object at $T^{\prime}, p_{d} \neq p_{d}^{\prime}$. Then, by Lemma 6and 31. $p_{d}^{\prime}$ was a parent of node $_{d}$ and $p_{d}$ was a parent of node $e_{d}$ just before $T^{\prime}$ and, by the claim, $p_{d}^{\prime}$ and $p_{d}$ are both reachable just before $T^{\prime}$, contradicting Lemma 9 (since $p_{d} \neq p_{d}^{\prime}$ ). Thus, node . info is an Unflag object at $T^{\prime}$ and, by Observation 26. node .info is not set to a Flag object at any time before $T^{\prime}$.

Now, we show that node $e_{d}$.info is not set to some Flag object $I^{\prime} \neq I$ between $T^{\prime}$ and $T$. Let $\left\langle-, p_{d}^{\prime}\right.$, node $e_{d}$, $-,-,-\rangle$ be the result returned by the call to $\operatorname{search}\left(v a l_{d}^{\prime}\right)$ on line 45 that precedes the creation of $I^{\prime}$. By Lemma 6 and 31. $p_{d}$ was a parent of node $_{d}$ at all times between $T^{\prime}$ and $T$. By the claim, $p_{d}$ is reachable at all times 
between $T^{\prime}$ and $T$. If node .info is set to $I^{\prime}$ between $T^{\prime}$ and $T$, by Lemma 6 and 31, $p_{d}^{\prime}$ was a parent of node

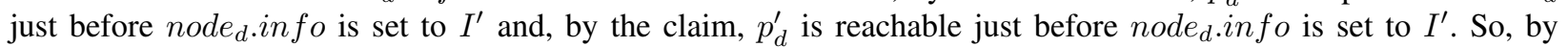
Lemma 9. $p_{d}=p_{d}^{\prime}$. In this case, $p_{d}=p_{d}^{\prime} \in F_{I^{\prime}}$. Thus, by Observation 26 and Lemma 22 . $p_{d}$. info $=I^{\prime}$ just before node $_{d}$.info is set to $I^{\prime}$ between $T^{\prime}$ and $T$. By Lemma 25, $p_{d}$.info $\neq I^{\prime}$ at all times between $T^{\prime}$ and $T$. Thus, node $_{d}$.info is not set to some Flag object $I^{\prime}$ between $T^{\prime}$ and $T$.

Next, we have the following lemma that shows when a leaf node is logically removed, other operations see that it is removed using the info field of the leaf node.

Lemma 41. Let I be a Flag object that is created at line 55 or 57 I.rmvLeaf is logically removed if and only if I.rmvLeaf.info $=I$ and I.pNode[0] is not a parent of I.oldChild[0].

Proof: First, we show that I.rmvLeaf.info $=I$ and I.pNode[0] is not a parent of I.oldChild[0] if I.rmvLeaf is logically removed. Let $T$ be the time when the first child CAS of $I$ is executed. By definition, I.rmvLeaf is logically removed at all times after T. By Observation 26, I.rmvLeaf.info is set to I before $T$. By Lemma 40, I.rmvLeaf.info $=I$ at all times after that. By Lemma 32 and 38, the first child CAS of $I$ successfully changes I.pNode[0].child[i] from I.oldChild[0] to I.newChild[0] at T. By Lemma 28 . I.oldChild $[0] \neq$ I.newChild $[0]$. By Lemma 38, I.pNode[0] is not a parent of I.oldChild $[0]$ after T. Thus, at all times after time T, I.rmvLeaf.info $=I$ and I.pNode[0] is not a parent of I.oldChild [0].

Now, we show that I.rmvLeaf is logically removed if I.rmvLeaf.info $=I$ and I.pNode[0] is not a parent of I.oldChild[0]. Suppose I.rmvLeaf.info $=I$ and I.pNode[0] is not a parent of I.oldChild[0] at some time T. Then $I$ was created before $T$, and during the search that precedes the creation of $I$, I.oldChild $[0]$ was a child of I.pNode[0] Thus, some child CAS changed I.pNode[0].child $[i]$ from I.oldChild $[0]$ to a different value before $T$. We argue that this must have been a child CAS of $I$, which means that I.rmvLeaf is logically removed at time $T$. Let $\left\langle-, p_{i}\right.$, node $\left._{i},-,-,-\right\rangle$ be the result returned by the call to the search operation on line 47 that precedes the creation of $I$. Then, I.pNode $[0]=p_{i}$, I.oldChild $[0]=$ node $_{i}$ and $p_{i} \in F_{I}$ by Observation 24. By Lemma 31, no child CAS of another Flag object $I^{\prime} \neq I$ changes $p_{i}$.child between the time when $p_{i}$.info is read for the last time during the search operation that precedes the creation of $I$.

Definition 42. A node is logically in the trie at time $T$ if the node is reachable at $T$ and the node is not logically removed at $T$.

Here, we show when the search operation terminates, all post-conditions of the search operation are satisfied.

Lemma 43. Each call to the search operation that terminates satisfies its post-conditions.

Proof: Assume search(val) returns $\langle g p$, p, node, gpInfo, pInfo, rmvd $\rangle$. Lemma 6 shows the first four post-conditions of the search operation are satisfied. We prove the last two here.

If rmvd is true, we prove that node is logically removed at line 124 Let $I$ be the value of node.info at line 84 If rmvd is set to true at line 84, node is a leaf node whose info field is a Flag object at line 84 and node.info.pNode[0] is not a parent of node.info.oldChild $[0]$ at line 124 . By Lemma 40 node.info $=I$ at all times after line 84 . Thus, node is logically removed at line 124 by Lemma 41.

If $r m v d$ is false, we prove that node is logically in the trie at some time during the search operation. By Lemma 36. node is reachable at some time $T$ between the beginning of the search and the time the search visits node. If node is an internal node, then it is logically in the trie at $T$. Otherwise, node is a leaf node. Since rmvd is false, line 123 or 124 returns false. If line 123 returns false, then when node.info is read on line 84 it is not flagged. This occurs after node is visited at line 82 and therefore after $T$. By Observation 26, node is not flagged at $T$, so node is logically in the trie at $T$ by Lemma 41 . If line 124 returns false, then node $=$ I.rmvLeaf (since the test at line 123 failed) and I.pNode[0] is a parent of I.oldChild[0] at line 124. By Lemma 41. node is not logically removed at line 124. Therefore, node is not logically removed at $T$.

Lemma 44. Assume search(val) returns $\langle$-, -, node, -, -, rmvd $\rangle$. If node is not a leaf node whose label is val, or rmvd is true, there is a time during the search operation when no leaf node whose label is val is logically in the trie.

Proof: First, consider the case where node is not a leaf node whose label is val. Let $p$ be the last internal node visited by $\operatorname{search}(v a l)$ such that p.label is a prefix of val. Let $i$ be the index such that $(p$.label). $i$ is a prefix 
of val. Let $x$ be the child of $p$ that the search operation visits. By definition of $p, x$ is not a leaf node whose label is val. By Lemma 36, there is a time during $\operatorname{search}(v a l)$ that $p . c h i l d[i]=x$ and $p$ and $x$ are both reachable. By Invariant 7, at that time, no leaf node whose label is val is reachable.

Now, consider the case where node is a leaf node whose label is val and rmvd is true. By Lemma 43, node is logically removed at some time during $\operatorname{search}(\mathrm{val})$. Let $T$ be the first time that node is logically removed. Then, the search operation executes line 124 after $T$. By Lemma 41, node.info is a Flag object $I$ at $T$. Then, $I$ is created at line 55 or 57 Let $p$ be the second last node visited by search(val). By Lemma 36 , there is a time $T^{\prime}$ during $\operatorname{search}(v a l)$ that $p . c h i l d[i]=$ node and $p$ and node are both reachable. At $T^{\prime}$, no other leaf node whose label is val is reachable by Invariant 7 . We consider two cases.

Case 1: $T$ is before $T^{\prime}$. Since node is logically removed at all times after $T$, node is logically removed at $T^{\prime}$. So, no leaf node whose label is val is logically in the trie at $T^{\prime}$.

Case 2: $T$ is between $T^{\prime}$ and the time when the search operation executes line 124. By the definition, the first child CAS of $I$ is performed at $T$. By Lemma 40, node.info $=I$ at all times after $T$. Let $\left\langle g p_{d}, p_{d}\right.$, node $_{d},-,-$, $-\rangle$ be the result returned by the call to the search operation on line 45 that precedes the creation of $I$ and $\left\langle-, p_{i}\right.$, node $\left._{i},-,-,-\right\rangle$ be the result returned by the call to the search operation on line 47 that precedes the creation of $I$. By the pseudo-code and Observation 26, I.rmvLeaf $=$ node $_{d}=$ node.

Since $I$ is created at line 55 or 57, $p_{d} \in F_{I}-U_{I}$ and $g p_{d} \in F_{I}$. By Corollary 39 $p_{d}$.info $=I$ and $g p_{d}$.info $=I$ just before $T$. By Lemma 6 and 31 , $g p_{d} . \operatorname{child}\left[i_{g p}\right]=p_{d}$ and $p_{d} . \operatorname{child}\left[i_{p}\right]=$ node just before $T$ for some $i_{g p}$ and $i_{p}$. Since $g p_{d} \in F_{I}$ and $g p_{d}$ was reachable at some time during the search operation by Lemma 36, $g p_{d}, p_{d}$ and node are reachable just before $T$ by Corollary 34 By the pseudo-code, $p_{i}$.child is changed at $T$. Now, we show that $g p_{d} \cdot \operatorname{child}\left[i_{g p}\right]$ and $p_{d} \cdot \operatorname{child}\left[i_{p}\right]$ are not changed at $T$. In this case, since $p_{d} \neq$ node $e_{i}, g p_{d} . c h i l d\left[i_{g p}\right]$ is not changed from $p_{d}$ to other value at $T$. Also, since $p_{d} \neq p_{i}, p_{d} \cdot \operatorname{child}\left[i_{p}\right]$ is not changed at $T$. So, just after $T$, $g p_{d} . \operatorname{child}\left[i_{g p}\right]=p_{d}$ and $p_{d}$.child $\left[i_{p}\right]=$ node and $g p_{d}$ is reachable since $g p_{d} \notin F_{I}-U_{I}$. By Invariant 7 , no other leaf node whose label is $\mathrm{val}$ is reachable just after $T$. Since node is logically removed at $T$, no leaf node whose label is val is logically in the trie just after $T$.

Now, we define a set that represents all non-empty leaf nodes that are logically in the trie at the same time.

Definition 45. We define the set activeValues at time $T$ to be the set of all values contained in leaf nodes that are logically in the trie at time $T$.

Since the children of root are initially two leaf nodes whose labels are $00 \ldots 0$ and $11 \ldots 1$, activeValues is initially $\{00 \ldots 0,11 \ldots 1\}$. By the definition of logically in the trie, activeValues can be changed only by successful child CAS steps.

A find $(v a l)$ operation that returns true executes a $\operatorname{search}(v a l)$ that returns $\langle-,-$, node, -, -, rmvd $\rangle$ where node is a leaf node whose label is val and rmvd is false. By the last post-condition of search, there is a time during the search when node is logically in the trie, so val $\in$ activeValues at that time. This is the linearization point of $\operatorname{search}(v a l)$ and find $(v a l)$ that returns true.

A find $(\mathrm{val})$ operation that returns false executes a $\operatorname{search}(\mathrm{val})$ that returns $\langle-,-$, node,,,---$\rangle$ where node is not a leaf node whose label is val, or rmvd is true. By Lemma 44, there is a time during the search when no leaf node whose label is val is logically in the trie, so val $\notin$ activeValues at that time. This is the linearization point of $\operatorname{search}(\mathrm{val})$ and find $(\mathrm{val})$ that returns false.

\section{E. Correctness of Update Operations}

In this section, we show that the update operations behave correctly. First, we show that if a child CAS of an update is performed, the operation returns true.

Lemma 46. If an update operation creates a Flag object I and a child CAS of I is performed, then the update operation returns true (unless it crashes).

Proof: Let $o p$ be the update operation that created I. Assume that, a child CAS of $I$ on I.pNode $[i]$ is performed and $o p$ does not crash. We show that $o p$ returns true. After creating $I$, op calls help $(I)$ at line 32, 41 or 71. There are two different cases inside help $(I)$ depending on the value of the doChildCAS variable at line 93 . 
Case 1: doChildCAS is true at line 93. So, op sets I.flagDone to true at line 94. By Observation 19, I. flagDone is not set to false after that. Since I. flagDone is true at line 99 , the call to help $(I)$ by op returns true at line 102 So, op returns true just after help $(I)$ returns.

Case 2: $o p$ sets doChildCAS to false on line 91 at time $T$. So, node.info $\neq I$ at $T$ for some node $\in F_{I}$. Before $T$, op tries to set node.info to $I$ using a flag CAS of $I$. So, the first flag CAS of $I$ on node is executed before $T$. By Corollary 39 and Lemma 16, this first flag CAS of $I$ on node succeeds before T. Since node.info $\neq I$ at $T$, node.info is set to $I$ and, then changed from $I$ to some other value before $T$. By Lemma 22, a child CAS of $I$ is performed before T. By Observation 20, I. flagDone is set to true before T. By Observation 19, I.flagDone is not set to false after that. Since I.flagDone is true at line 99 the call to help $(I)$ by op returns true at line 102 So, op returns true just after help $(I)$ returns.

By Lemma 46, we have the following corollary.

Corollary 47. Let I be a Flag object that is created by an update operation. If the update operation returns false, there is no child CAS of $I$.

During each loop iteration of an update operation, the update operation might create a new Flag object $I$. The following lemma shows if the update operation begins the next iteration of the loop, no child CAS of $I$ succeeds.

Lemma 48. Let I be a Flag object that is created during a loop iteration of an update operation. If the update operation begins the next iteration of the loop, no child CAS of I is ever performed.

Proof: Assume that an update operation op creates a Flag object $I$ during a loop iteration and begins the next iteration of the loop. After creating $I, o p$ calls help $(I)$ at line 32, 41 or 71. Since $o p$ does not return true at the end of the loop iteration after help $(I)$ returns, help $(I)$ returns false. By Lemma 46, there is no child CAS of $I$.

Let $I$ be a Flag object that is created at line 55 or 57. Since the condition at line $\mathrm{F}$ that precedes the creation of $I$ is satisfied, I.oldChild $[0] \neq$ I.oldChild[1]. So, by Lemma 38, we have the following Corollary.

Corollary 49. Let I be a Flag object in which I.pNode has two elements. Then, the child CAS of I on I.pNode[0] and I.pNode[1] cannot succeed on the same location.

Lemma 50. Let I be a Flag object that is created at line 59 Let $\left\langle-, p_{d}\right.$, node $\left.d_{d},-,-,-\right\rangle$ be the result returned by the call to search $\left(v^{2} l_{d}\right)$ on line 45 and $\left\langle-, p_{i}\right.$, node $\left.i_{i},-,-,-\right\rangle$ be the result returned by the call to search $\left(v^{2} l_{i}\right)$ on line 47 that precedes the creation of I. Then, if there is a successful child CAS of I at time $T, p_{d}=p_{i}$.

Proof: In this case, node $_{i}=$ node $_{d}$. By Lemma 30, $T$ is the first child CAS of $I$. By Lemma 6 and $31, p_{d}$ was a parent of node $e_{d}$ at all times between the time when $\operatorname{search}\left(v a l_{d}\right)$ reads $p_{d}$.info and $T$. Since $p_{d} \in F_{I}$, by Corollary 39. $p_{d}$.info $=I$ just before $T$. By Lemma 36 $p_{d}$ was reachable at some time during $\operatorname{search}\left(v a l_{d}\right)$. By Lemma 25, $p_{d}$ is not marked between the time when $\operatorname{search}\left(v a l_{d}\right)$ reads $p_{d}$.info and T. By Corollary 34, $p_{d}$ is reachable at all times between the time when $\operatorname{search}\left(v a l_{d}\right)$ returns and $T$. By Lemma 36, there is a time during $\operatorname{search}\left(v a l_{i}\right)$ that $p_{i}$ is a parent of node $_{i}=$ node $_{d}$ and $p_{i}$ is reachable. So, at that time, $p_{d}$ and $p_{i}$ are both parent of node and both reachable. By Lemma 9, $p_{i}=p_{d}$.

Lemma 51. Consider a Flag object I. Let $\langle g p$, $p$, node, gpInfo, $p I n f o$, - $\rangle$ be the result returned by the call to search(val) on line $23,36,45$ that precedes the creation of $I$. If I is created by a replace operation, let $\left\langle-, p_{i}\right.$, node $_{i},-$, pInfo $\left._{i},-\right\rangle$ be the result returned by the call to search $\left(v_{a l}\right)$ on line 47 that precedes the creation of $I$. Then, if there is a successful child CAS of I on I.pNode[0] at time T,

- $p$ and node are reachable just before $T$, and

- $p_{i}$ and node ${ }_{i}$ are reachable just before $T$ (if I is created by a replace operation).

Proof: To prove the lemma, first we show $p$ and node are reachable just before $T$. Since $I$ is created by an update operation, $p \in F_{I}$ and $T$ is the first child CAS of $I$ by Lemma 32. By Corollary 39, p.info $=I$ just before $T$. By Lemma 36 $p$ was reachable at some time during $\operatorname{search}(v a l)$. Since $p$ is not marked before $T$, by Corollary 34. $p$ is reachable just before $T$. By Lemma $6 p$ is a parent of node at some time during $\operatorname{search}(\mathrm{val})$. By the pseudo-code, $p \in F_{I}$. By Lemma 31, $p$ is a parent of node just before $T$. Since $p$ is reachable just before $T$, node is also reachable just before $T$. 
For the rest of the proof, assume $I$ is created by a replace operation. Now, we show $p_{i}$ and node $_{i}$ are reachable just before $T$. If $I$ is created at line 59, by Lemma 50, $p=p_{i}$ and $p_{i} \in F_{I}$. If $I$ is created at any other line inside a replace operation, by the pseudo-code, $p_{i} \in F_{I}$. By Corollary 39, $p_{i}$ info $=I$ just before $T$. By Lemma 36, $p_{i}$ was reachable at some time during $\operatorname{search}\left(v a l_{i}\right)$. Since $p_{i}$ is not marked before $T$, by Corollary $34, p_{i}$ is reachable just before $T$. By Lemma $6 p_{i}$ is a parent of $\operatorname{node}_{i}$ at some time during $\operatorname{search}\left(v a l_{i}\right)$. By Lemma 31, $p_{i}$ is a parent of node $_{i}$ just before $T$ since $p_{i} \in F_{I}$. Since $p_{i}$ is reachable just before $T$, node $e_{i}$ is also reachable just before $T$.

Lemma 52. Let $I$ be a Flag object that is created at line 55 or 57 and $\left\langle g p_{d}, p_{d}\right.$, node $\left.e_{d},-,-,-\right\rangle$ be the result returned by the call to search $\left(v_{a l}\right)$ on line 45 that precedes the creation of I. Then, if there are a successful child CAS of I on I.pNode[0] at T and a successful child CAS of I on I.pNode[1] at $T^{\prime}, p_{d}$ and node are reachable $^{2}$ at all times between $T$ and $T^{\prime}$ and $p_{d}$ and node $e_{d}$ become unreachable immediately after $T^{\prime}$.

Proof: First, we show that $p_{d}$ and node $e_{d}$ are reachable at all times between $T$ and $T^{\prime}$. By Lemma 51 and 51 . node $_{d}$ and $p_{d}$ are reachable just before $T$. In this case, $g p_{d}$ and $p_{d}$ are in $F_{I}$. By Lemma $6, g p_{d}$ is a parent of $p_{d}$ at some time during search $\left(v a l_{d}\right)$ and $p_{d}$ is a parent of node $e_{d}$ at some time during $\operatorname{search}\left(v a l_{d}\right)$. By Lemma 31 and Corollary 49, $g p_{d}$ is a parent of $p_{d}$ and $p_{d}$ is a parent of node $e_{d}$ just before $T^{\prime}$ (since $p_{i} \neq p_{d}$ ). By Corollary 39 and Lemma 22, $g p_{d}$.info $=I$ just before $T^{\prime}$ and $g p_{d}$ is not marked just before $T^{\prime}$. By Corollary $34 . g p_{d}$ is reachable just before $T^{\prime}$. So, $p_{d}$ and node $_{d}$ are reachable just before $T^{\prime}$. Thus, by Lemma 37, $p_{d}$ and node $e_{d}$ are reachable at all times between $T$ and $T^{\prime}$.

Now, we show that $p_{d}$ and node $e_{d}$ become unreachable immediately after $T^{\prime}$. By Lemma 35, $p_{d}$ becomes unreachable at $T^{\prime}$. By Lemma 9. $p_{d}$ is the only reachable parent of node $e_{d}$ just before $T^{\prime}$. Thus, node $e_{d}$ also becomes unreachable at $T^{\prime}$.

Lemma 53. Consider a Flag object I. Let $\langle$-, p, node, -, pInfo, rmvd $\rangle$ be the result returned by the call to the search operation on line 23,36 or 45 that precedes the creation of I. If the first successful child CAS of I is at time $T$, then node is logically in the trie just before $T$.

Proof: By Lemma 51, node is reachable just before $T$. We show that node is not logically removed at any time before $T$.

To derive a contradiction, assume node becomes logically removed at $T^{\prime}$ before $T$. Let $I^{\prime} \neq I$ be the Flag object such that node is logically removed by the first child CAS of $I^{\prime}$ at $T^{\prime}$. Then, $I^{\prime}$ is created at line 55 or 57 Let $\langle-$, $p_{i}$, node $\left._{i},-,-,-,-,-\right\rangle$ be the result returned by the call to the search operation on line 45 that precedes the creation of $I^{\prime}$. Since $I^{\prime}$.rmvLeaf is set to node $e_{i}$, node $=$ node $_{i}$. Since node and $p_{i}$ become unreachable immediately after the successful child CAS of $I^{\prime}$ on $I^{\prime}$.pNode[1] (by Lemma 52, node and $p_{i}$ do not become reachable after that (by Lemma 37). But, node and $p_{i}$ are reachable just before $T$ (by Lemma 51 and 51), so the successful child CAS of $I^{\prime}$ on $I^{\prime}$.pNode[1] does not occur before $T$. By Lemma 31 and Corollary 49, $p_{i}$ is a parent of node just before $T$ (since $T$ is between the first child CAS of $I^{\prime}$ on $I^{\prime} . p N o d e[0]$ and the first child CAS of $I^{\prime}$ on $I^{\prime}$.pNode[1]). By Lemma 6 and 31, $p$ is a parent of node just before $T$. By Lemma 51 $p$ is reachable just before $T$. By Lemma 9 $p_{i}=p$ (since both $p$ and $p_{i}$ are reachable parents of node just before $T$ ). Since $I^{\prime}$ is created at line 55 or 57, by the pseudo-code, $p=p_{i} \in F_{I^{\prime}}$. By Corollary 39 p.info $=I^{\prime}$ just before $T$ (that is between the first child CAS of $I^{\prime}$ on $I^{\prime} . p N o d e[0]$ and the first child CAS of $I^{\prime}$ on $I^{\prime}$.pNode[1]), contradicting the fact that $p$.info $=I \neq I^{\prime}$ just before $T$ by Lemma 25 .

1) Correctness of the Insert Operation: In this section, we show how the set activeValues is changed by insert operations and, then we show how to linearize insert operations.

Lemma 54. If insert(val) returns false, there is a time during the insert(val) when val $\in$ activeValues.

Proof: Assume insert(val) returns false. Let $\langle-$, -, node, -, -, rmvd $\rangle$ be the result returned by the last call to $\operatorname{search}(\mathrm{val})$ just before insert $(\mathrm{val})$ returns. Since insert(val) returns false at line 24, keyInTrie(node, val, rmvd) returns true at line 24. Then, node is a leaf node containing val and rmvd is false. By Lemma 43, node is logically in the trie at some time during $\operatorname{search}(v a l)$. So, val $\in$ activeValues at that time.

Let $I$ be a Flag object created by insert $(\mathrm{val})$. By Corollary 47 , there is no successful child CAS of $I$ if insert ( $v a l)$ returns false. So, activeValues is not changed by a child CAS of $I$.

Let $I$ be a Flag object that is created during a loop iteration of insert $(v a l)$. If a child CAS of $I$ succeeds, by Lemma 46, insert $(\mathrm{val})$ returns true at the end of the loop iteration unless it crashes. If a child CAS of $I$ succeeds, 
we say insert $(\mathrm{val})$ is successful. By Lemma 30 and 32, only the first child CAS of $I$ succeeds. In the following lemma, we show how the successful insert operations change activeValues.

Lemma 55. Let I be a Flag object that is created by insert(val). Assume a child CAS of I succeeds at time T. Let $s$ be the set activeValues just before $T$ and let $s^{\prime}$ be the set activeValues just after T. Then, val $\notin s$ and $s^{\prime}=s \cup\{v a l\}$

Proof:

Let $\langle-, p$, node, -, -, rmvd $\rangle$ be the result returned by the call to $\operatorname{search}(v a l)$ that precedes the creation of $I$. Then, $p \in F_{I}$. By Lemma 6. p.label is a prefix of val and if node is an internal node, node.label is not a prefix of $\mathrm{val}$. Since insert $(\mathrm{val})$ does not return false at line 24 before the creation of $I$ by Corollary 47, either node is not a leaf node whose label is val or rmvd is true. If node is not a leaf node whose label is val, since $p$ is parent of node just before $T$ (by Lemma 6 and 31p and $p$ is reachable just before $T$ (by Lemma 51), $p$ is not an ancestor of a leaf node whose label is val just before T. If node is a leaf node whose label is val and rmvd is true, by Lemma 43, node is logically removed at some time during search $(v a l)$, so node is logically removed just before $T$. Since $p$ is a parent of node just before $T$ and $p$ is reachable just before $T$, no leaf node whose label is val is logically in the trie just before $T$ by Invariant 7 . Thus, val $\notin s$.

The successful child CAS of I changes an element of I.pNode[0].child from I.oldChild[0] to I.newChild[0]. When $I$ is initialized, I.pNode[0] and I.oldChild[0] are set to $p$ and node respectively. The successful child CAS of $I$ changes an element of p.child from node to I.newChild[0] at time $T$. When $I$ is initialized, I.newChild $[0]$ is set to the new internal node created at line 121 . The non-empty elements of the child field of the new internal node are a new copy of node and a new leaf node whose label is val.

If node is a leaf node, since $p$ is reachable just before $T$ (by Lemma 51, just after $T$, node is unreachable and the new leaf node and the new copy of node are reachable. So, $s^{\prime}=s \cup\{$ val $\}$.

If node is an internal node, node $\in F_{I}$ by the pseudo-code and, by Lemma 31. no child CAS changes node.child between the time when node.info is read for the last time on line 25 and $T$. Since the new copy of node is made at line 26 between the time when node.info is read for the last time on line 25 and $T$, node.child just before $T$ is the same as the children of the new copy of node just after $T$. By Lemma 51 $p$ is reachable just before $T$. Just after $T$, node is unreachable and the new leaf node and the new copy of node are reachable. Also, all children of node just before $T$ are reachable just after $T$. So, activeValues $=s \cup\{$ val $\}$, just after $T$.

An insert $(\mathrm{val})$ returns false executes a search $(\mathrm{val})$ that returns $\langle-,-$, node,,,$-- \mathrm{rmvd}\rangle$ where node is a leaf node whose label is val and rmvd is false. By the last post-condition of search, there is a time during the search when node is logically in the trie, so val $\in$ activeValues at that time. This is the linearization point of $\operatorname{search}(\mathrm{val})$ and insert $(\mathrm{val})$ that returns false. By Corollary 47, if insert( $\mathrm{val}$ ) returns false, there is no child CAS of $I$ where $I$ is created by insert $(\mathrm{val})$.

If the first child CAS of $I$ that is created by insert $(\mathrm{val})$ occurs, insert $(\mathrm{val})$ is linearized at that child CAS of $I$. By Lemma 32, the first child CAS of $I$ succeeds and by Lemma 30, no other child CAS of $I$ succeeds. By Lemma 55. val $\notin$ activeValues just before the first child CAS of $I$ and val is added to activeValues just after the first child CAS of $I$. By Lemma 46, if the first child CAS of $I$ occurs, insert(val) returns true (unless it crashes).

If insert $(\mathrm{val})$ does not return false and the first child CAS of $I$ that is created by insert( $\mathrm{val}$ ) does not occur, no linearization point is assigned to insert $(\mathrm{val})$.

2) Correctness of the Delete Operation: In this section, we show how the set activeValues is changed by delete operations and, then we show how to linearize delete operations.

Lemma 56. If delete(val) returns false, there is a time during the delete(val) when val $\notin$ activeValues.

Proof: Assume delete $(v a l)$ returns false at line 37 Let $\langle-$, -, node, -, - rmvd $\rangle$ be the result returned by the last call to $\operatorname{search}(\mathrm{val})$ before delete $(\mathrm{val})$ returns false. Since delete( $\mathrm{val})$ returns false at line 37. keyInTrie (nod, val,rmvd) returns false at line 37. Then, node is not a leaf node whose label is val or rmvd is true. By Lemma 44 , there is a time during $\operatorname{search}(v a l)$ when there is no leaf node that has label val is logically in the trie. So, val $\notin$ activeValues at that time.

Let $I$ be a Flag object created by delete( $v a l)$. By Corollary 47, there is no successful child CAS of $I$. So, activeValues is not changed by a child CAS of $I$. 
Let $I$ be a Flag object that is created by delete( $v a l)$ during a loop iteration. If a child CAS of $I$ on I.pNode[0] succeeds, by Lemma 46, delete $(\mathrm{val})$ returns true at the end of the loop iteration unless it crashes. If a child CAS of $I$ on I.pNode[0] succeeds, we say delete $(\mathrm{val})$ is successful. By Lemma 30 and 32, only the first child CAS of $I$ succeeds. In the following lemma, we show how the successful delete operations change activeValues.

Lemma 57. Let I be a Flag object that is created by delete(val). Assume a child CAS of I succeeds at time T. Let $s$ be the set activeValues just before $T$ and let $s^{\prime}$ be the set activeValues just after T. Then, val $\in s$ and $s^{\prime}=s-\{v a l\}$

Proof: Let $\langle g p, p, n o d e,-,-, r m v d\rangle$ be the result returned by the call to search $(v a l)$ that precedes the creation of $I$. Since delete $(\mathrm{val})$ does not return false at line 37 by Corollary 47 , node is a leaf node whose label is val and rmvd is false. By Lemma 53, node is logically in the trie just before $T$, so val $\in s$.

The successful child CAS of I changes an element of I.pNode[0].child from I.oldChild[0] to I.newChild[0]. Now, we show that $s^{\prime}=s-\{v a l\}$. The nodeSibling variable is set to an element of p.child at line 38 . When $I$ is initialized, I.pNode[0], I.oldChild[0] and I.newChild[0] are set to gp, $p$ and nodeSibling respectively at line 40 Let $T^{\prime}$ be the time when p.info is read for the last time during the $\operatorname{search}(\mathrm{val})$ that precedes the creation of $I$. By Lemma 31, since $p \in F_{I}$, p.child is not changed between $T^{\prime}$ and $T$. Since $p$ is a parent of node after $T^{\prime}$ during search $(\mathrm{val})$ (by Lemma 6), node and nodeSibling are children of $p$ at all times between $T^{\prime}$ and $T$. The child CAS of $I$ changes an element of gp.child from $p$ to nodeSibling at $T$. By Lemma 51 $p$ is reachable just before $T$. So, just before $T$, node and nodeSibling are also reachable. By Lemma 9 , no other leaf node whose label is val is reachable just before $T$.

In this case, $g p \notin F_{I}-U_{I}$. By Lemma 25 and Corollary 34, $g p$ is reachable just before $T$. So, just after $T, p$ and node are not reachable, but $g p$ and nodeSibling are reachable. If nodeSibling is an internal node, all elements of its child field that are reachable just before $T$ are reachable just after $T$. Thus, $s^{\prime}=s-\{v a l\}$.

A delete $(\mathrm{val})$ returns false executes a $\operatorname{search}(\mathrm{val})$ that returns $\langle-,-$, node, -, -, rmvd $\rangle$ where node is not a leaf node whose label is val, or rmvd is true. By Lemma 44, there is a time during the search when no leaf node whose label is val is logically in the trie, so val $\notin$ activeValues at that time. This is the linearization point of $\operatorname{search}(\mathrm{val})$ and delete $(\mathrm{val})$ that returns false. By Corollary 47 , if delete( $\mathrm{val})$ returns false, there is no child CAS of $I$ where $I$ is created by delete $(\mathrm{val})$.

If the first child CAS of $I$ that is created by delete $(\mathrm{val})$ occurs, delete $(\mathrm{val})$ is linearized at that child CAS of $I$. By Lemma 32, the first child CAS of $I$ succeeds and by Lemma 30, no other child CAS of $I$ succeeds. By Lemma 57. val $\in$ activeValues just before the first child CAS of $I$ and $v a l$ is removed from activeValues just after the first child CAS of $I$. By Lemma 46, if the first child CAS of $I$ occurs, delete(val) returns true (unless it crashes).

If delete $(\mathrm{val})$ does not return false and the first child CAS of $I$ that is created by delete( $\mathrm{val})$ does not occur, no linearization point is assigned to delete ( $\mathrm{val}$ ).

3) Correctness of the replace operation: In this section, we show how the set activeValues is changed by replace operations and, then we show how to linearize replace operations.

Lemma 58. If replace $\left(v_{a l}, v_{a l}\right)$ returns false, there is a time during replace $\left(v_{a l}, v_{a l}\right)$ when $v_{a l} \notin$ activeValues or val $_{i} \in$ activeValues.

Proof: Assume replace $\left(v l_{d}, v_{i} l_{i}\right)$ returns false. Then, we have two cases according to what line returns false.

Case 1: replace $\left(v a l_{d}, v a l_{i}\right)$ returns false at line 46. Let $\left\langle-,-\right.$, node $\left._{d},-,-, r m v d_{d}\right\rangle$ be the result returned by the last call to search $\left(v_{a l} l_{d}\right)$ on line 45. So, keyInTrie node $_{d}$, val $_{d}$, moved $_{d}$ ) returns false at line 46 Then, node $e_{d}$ is not a leaf node containing $v a l_{d}$ or $r m v d_{d}$ is true. By Lemma 44, there is a time during search $\left(v a l_{d}\right)$ when there is no leaf node that has label $v_{a l}$ and is logically in the trie. So, val $\notin$ activeValues at that time.

Case 2: replace $\left(v a l_{d}, v a l_{i}\right)$ returns false at line 48 Let $\left\langle-,-\right.$, node $\left._{i},-,-r m v d_{i}\right\rangle$ be the result returned by the last call to $\operatorname{search}\left(v a l_{i}\right)$ on line 47 before replace $\left(v a l_{d}, v a l_{i}\right)$ returns false. So, keyInTrie $\left(\right.$ node $\left._{i}, v a l_{i}, r m v d_{i}\right)$ returns true. Then, node $e_{i}$ is a leaf node containing $v a l_{i}$ and $r m v d_{i}$ is false. By Lemma 43, node $e_{i}$ is logically in the trie at some time during $\operatorname{search}\left(v_{a l}\right)$. So, $v_{a l} \in$ activeValues at that time.

Let $I$ be a Flag object created by replace $\left(v a l_{d}, v a l_{i}\right)$. By Corollary 47, there is no successful child CAS of $I$. So, activeValues is not changed by a child CAS of $I$.

Let $I$ be a Flag object that is created by replace $\left(v_{a l}, v_{d} l_{i}\right)$ during a loop iteration. If, for all $i$, a child CAS of $I$ on I.pNode $[i]$ succeeds, by Lemma 46 replace $\left(v a l_{d}, v a l_{i}\right)$ returns true at the end of the loop iteration unless it 
crashes. If a child CAS of $I$ on I.pNode[0] succeeds, we say replace $\left(v_{a l} l_{d}, v_{a l}\right)$ is successful. By Lemma 30 and 32 . only the first child CAS of $I$ on I.pNode[0] succeeds.

There are different cases of replace $\left(v a l_{d}, v a l_{i}\right)$ depending on what line creates $I$. The general case of replace $\left(v a l_{d}, v a l_{i}\right)$ is when $I$ is created at line 55 or 57. The special cases of replace $\left(v a l_{d}, v a l_{i}\right)$ are when $I$ is created at line 59. 64 or 70 . First, we show how successful replace operations that perform the general case change activeValues.

Lemma 59. Let I be a Flag object that is created by replace $\left(v_{a} l_{d}, v_{i l}\right)$ at line 55 or 57 Assume a child CAS of I on I.pNode[0] succeeds at time $T_{0}$ and a child CAS of I on I.pNode[1] succeeds at time $T_{1}$. Let $s_{0}$ be the set activeValues just before $T_{0}$, let $s_{0}^{\prime}$ be the set activeValues just after $T_{0}$, let $s_{1}$ be the set activeValues just before $T_{1}$ and let $s_{1}^{\prime}$ be the set activeValues just after $T_{1}$. Then, val $l_{d} \in s_{0}, v a l_{i} \notin s_{0}, s_{0}^{\prime}=s_{0} \cup\left\{v a l_{i}\right\}-\left\{v a_{d}\right\}$ and $s_{1}^{\prime}=s_{1}$.

Proof: Let $\left\langle-, p_{i}\right.$, node $\left._{i},-,-, r m v d_{i}\right\rangle$ be the result returned by the call to $\operatorname{search}\left(v a l_{i}\right)$ on line 47 that precedes the creation of $I$. When $I$ is initialized, I.pNode[0], I.oldChild[0] and I.newChild[0] are set to $p_{i}$, node $e_{i}$ and the new node that is created at line 53 . The child CAS of $I$ on I.pNode[0] at $T_{0}$ behaves in the same way as a successful child CAS of an $\operatorname{insert}\left(\right.$ val $\left._{i}\right)$ operation. By a similar argument to Lemma 55, only node $e_{i}$ becomes unreachable just after $T_{0}, v a l_{i} \in s_{0}^{\prime}$ and $v a l_{i} \notin s_{0}$.

Now, we show that $v a l_{d} \in s_{0}$. Let $\left\langle g p_{d}, p_{d}\right.$, node $\left._{d},-,-, r m v d_{d}\right\rangle$ be the result returned by the call to $\operatorname{search}\left(v a l_{d}\right)$ on line 45 that precedes the creation of $I$. Since replace $\left(v a l_{d}, v a l_{i}\right)$ does not return false at line 46 node $e_{d}$ is a leaf node whose label is $v_{a l}$ and $r m v d_{d}$ is false. By Lemma 53, node $d_{d}$ is logically in the trie just before $T_{0}$, so $v a l_{d} \in s_{0}$. Next, we show that, $v a l_{d} \notin s_{0}^{\prime}$.

By the pseudo-code, I.rmvLeaf is node $_{d}$. By the definition of logically removed, node in $_{\text {in }}$ logically removed just after $T_{0}$ and node $e_{d}$ is not logically in the trie at any time after $T_{0}$. Since node $e_{d}$ is reachable just before $T_{0}$ (by Lemma 51, no other leaf node whose label is $v_{a l}$ is reachable just before $T_{0}$ by Lemma9. Since the condition at line $\mathrm{F}$ is true, node $_{d} \neq$ node $_{i}$. So, node $_{d}$ is reachable just after $T_{0}$ (since I.oldChild $[0] \neq$ node $_{d}$ ). So, no other leaf node whose label is $v_{a l}$ is reachable just after $T_{0}$. So, $s_{0}^{\prime}=s_{0}-\left\{v a l_{d}\right\} \cup\left\{v l_{i}\right\}$ (since $v_{a l} \notin s_{0}$ and $v_{a l} \in s_{0}^{\prime}$ ).

Now, we show $s_{1}=s_{1}^{\prime}$. By the definition of logically removed, node is logically removed at all times after $T_{0}$. By Lemma 51. node ${ }_{d}$ is reachable just before $T_{1}$. By Lemma 9, no other leaf node whose label is $v^{2} l_{d}$ is reachable just before $T_{1}$. So, val $_{d} \notin s_{1}$.

Let nodeSibling be the node that is read as an element of $p_{d}$.child at line 50 Since $I$ is created at line 55 or 57. I.pNode[1], I.oldChild[1] and I.newChild[1] are set to $g p_{d}, p_{d}$ and nodeSibling, respectively. The child CAS of $I$ on I.pNode[1] is the same as a successful child CAS of $I^{\prime}$ where $I^{\prime}$ is created by delete $\left(v a l_{d}\right)$ at line 40 However, by the definition of logically removed, node $e_{d}$ is logically removed just after $T_{0}$. Since the condition

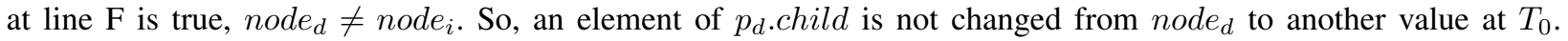
By a similar argument to Lemma 57, only node $e_{d}$ and $p_{d}$ become unreachable just after $T_{1}$ and no other leaf node whose label is $\mathrm{val}_{d}$ becomes reachable just after $T_{1}$. Since node ${ }_{d}$ is a leaf node whose label is $v_{a l}$, node ${ }_{d}$ is the only leaf node that becomes unreachable just after $T_{1}$ and $v a l_{d} \notin s_{1}, s_{1}=s_{1}^{\prime}$.

Next, we show how successful replace operations that perform special cases of the replace operation change activeValues.

Lemma 60. Let I be a Flag object that is created by replace $\left(v_{a l}, v_{a l}\right)$ at line 59,64 or 70 . Assume a child CAS of I succeeds at time T. Let $s$ be the set activeValues just before $T$ and let $s^{\prime}$ be the set activeValues just after T. Then, $v l_{d} \in s, v_{a l} \notin s$ and $s^{\prime}=s \cup\left\{v a l_{i}\right\}-\left\{v a l_{d}\right\}$.

Proof: Let $\left\langle g p_{d}, p_{d}, n o d e_{d},-,-, r m v d_{d}\right\rangle$ be the result returned by the call to search $\left(v a l_{d}\right)$ on line 45 that precedes the creation of $I$. Since replace $\left(v a l_{d}, v a l_{i}\right)$ did not return false at line 46, node $e_{d}$ is a leaf node whose label is $v a l_{d}$ and $r m v d_{d}$ is false. By Lemma 53 node $e_{d}$ is logically in the trie just before $T$. So, val $l_{d} \in s$.

Now, we show no leaf node containing $v a l_{i}$ is reachable just before $T$. Let $\left\langle-, p_{i}\right.$, node $\left.e_{i},-,-, r m v d_{i}\right\rangle$ be the result returned by the call to $\operatorname{search}\left(v_{a l}\right)$ on line 47 that precedes the creation of $I$. By Lemma 6, $p_{i} . l a b e l$ is a prefix of $v a l_{i}$ and if node $_{i}$ is an internal node, node $e_{i}$ label is not a prefix of $v a l_{i}$. So, by Invariant $7 p_{i}$ is the lowest internal node whose label is a prefix of $v_{a l} l_{i}$ that $\operatorname{search}\left(v a l_{i}\right)$ visits. By Lemma 6 $p_{i}$ is a parent of node $e_{i}$ at some time during $\operatorname{search}\left(v a l_{i}\right)$. By Lemma 31, $p_{i}$ is a parent of node $e_{i}$ just before $T$. By Lemma 51, $p_{i}$ is reachable just before $T$. 
Since replace $\left(v a l_{d}, v a l_{i}\right)$ did not return false at line 48, node $e_{i}$ is not a leaf node containing $v a l_{i}$ or $r m v d_{i}$ is true. If $r m v d_{i}$ is true, by Lemma 43, node $e_{i}$ is logically removed at some time during $\operatorname{search}\left(v a l_{i}\right)$. If node $_{i}$ is a leaf node whose label is $v a l_{i}$, since $r m v d_{i}$ is true, node $e_{i}$ is logically removed just before $T$. Since $p_{i}$ is a reachable parent of node $e_{i}$ just before $T, p_{i}$ is not an ancestor of any other leaf node containing $v_{a l}$ just before $T$. If node $_{i}$ is a leaf node and node $e_{i} . l a b e l \neq v a l_{i}$, since $p_{i}$ is a reachable parent of node $e_{i}$ just before $T$ and $p_{i} . l a b e l$ is a prefix of $v_{a l}$ (by Invariant 7), $p_{i}$ is not an ancestor of any leaf node containing $v a l_{i}$ just before $T$. If node $_{i}$ is an internal node, since $p_{i}$ is a reachable parent of node $e_{i}$ just before $T$ and node $e_{i}$.label is not a prefix of val $_{i}$ (by Lemma 6), $p_{i}$ is not an ancestor of any leaf node containing $v_{a l}$ just before $T$. Thus, no leaf node containing $v a l_{i}$ is logically in the trie just before $T$ and $v a l_{i} \notin s$.

The successful child CAS of I changes an element of I.pNode[0].child from I.oldChild[0] to I.newChild[0]. There are different special cases. We show that $s^{\prime}=s-\left\{v a l_{d}\right\} \cup\left\{v a l_{i}\right\}$ for each case.

Case 1: $I$ is created at line 59. Then, node $_{d}=$ node $_{i}$. When $I$ is initialized, I.pNode[0] I.oldChild[0] and I.newChild $[0]$ are set to $p_{d}$, node $_{i}$ and a new leaf node whose label is $v_{a l}$. Since the operation does not return at line 46 node $_{d}=$ node $_{i}$ is a leaf node and node $d_{d} . l a b e l=v_{a l}$. By Lemma 51, node ${ }_{i}$ is reachable just before $T$. By Lemma 9 no other leaf node containing $v a l_{d}$ is reachable just before $T$ (since node ${ }_{i}=$ node $_{d}$ ). So, $p_{d}$ and the new leaf node whose label is $\mathrm{val}_{i}$ are reachable just after $T$ and node $i=$ node $_{d}$ becomes unreachable at $T$. Since node $_{i}=$ node $_{d}$ is a leaf node whose label is val ${ }_{d}, s^{\prime}=s-\left\{v_{a l}\right\} \cup\left\{v_{a l} l_{i}\right\}$.

Case 2: $I$ is created at line 64. Let nodeSibling be an element of $p_{d}$.child that is read at line 50. Then, newNode be the node that is created at line 62. Then, newNode.child are initially a new leaf node whose label is $v^{2} l_{i}$ and nodeSibling. When I is created, I.pNode[0], I.oldChild $[0]$ and I.newChild[0] are set to $g p_{d}, p_{d}$ and newNode.

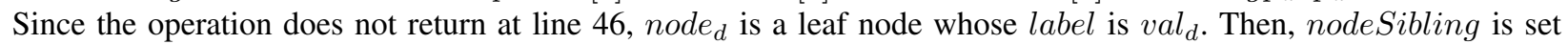
to an element of $p_{d}$.child that is not node $e_{d}$ at line 50 (by Invariant 7). So, node $e_{d}$ and nodeSibling are children of $p_{d}=$ node $_{i}$ just before $T$ (by Lemma 31. By Lemma 51, node $e_{d}$ is reachable just before $T$. Since node is a leaf node containing $\mathrm{val}_{d}$, by Lemma9 no other leaf node containing $\mathrm{val}_{d}$ is reachable just before $T$. By Lemma $9 g p_{d}$ is the only reachable parent of $p_{d}$ just before $T$ and $p_{d}$ is the only reachable parent of node $e_{d}$ just before $T$. At $T$,

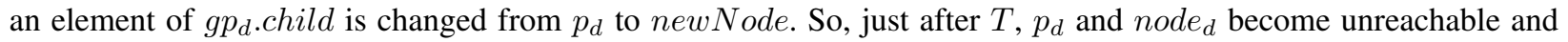
newNode and a new Leaf node whose label is $v^{2} l_{i}$ become reachable. Since nodeSibling is a child of newNode just after $T$, nodeSibling is reachable just after $T$. Thus, $s^{\prime}=s-\left\{v a l_{d}\right\} \cup\left\{v a l_{i}\right\}$.

Case 3: $I$ is created at line 70. Then, $g p_{d}=$ node $_{i}$. Let nodeSibling be an element of $p_{d}$.child that is read at line 50. pSibling be an element of $g p_{d}$ that is read at line 66 Then, newChild is the new node that is created at line 67 and newNode is the Internal node that is created at line 69. Initially, the children of newChild are nodeSibling and $p$ Sibling. Initially, the children of newNode are a new Leaf node whose label is val $_{i}$ and newChild. When $I$ is created, I.pNode[0], I.oldChild[0] and I.newChild[0] are set to $p_{i}$, node $e_{i}$ and newNode. Since $p_{i} \in F_{I}$, by Lemma 25. $p_{i}$ is not marked at all times between the last time $p_{i}$.info is read during $\operatorname{search}\left(v a l_{i}\right)$ and $T$. By Corollary $34 p_{i}$ is reachable at all times between the last time $p_{i}$.info is read during $\operatorname{search}\left(v a l_{i}\right)$ and $T$. By Lemma 6 and 31. $p_{i}$ is a parent of node $_{i}$ just before $T$. So, node $e_{i}$ is reachable just before $T$. Since $g p_{d} \in F_{I}$, the children of $g p_{d}=$ node $_{i}$ are $p_{d}$ and $p$ Sibling just before $T$ (by Lemma 31). Since $p_{d} \in F_{I}$, the children of $p_{d}$ are node $_{d}$ and nodeSibling just before $T$ (by Lemma 31. By Lemma 51 $p_{i}$, node $_{i}=g p_{d}, p_{d}, p$ Sibling, node $_{d}$ and nodeSibling are reachable just before $T$. Since node $_{d}$ is reachable just before $T$, no other leaf node containing $v a l_{d}$ is reachable just before $T$ (by Lemma 9p. By Lemma 9, $p_{i}$ is the only reachable parent of $g p_{d}=$ node $_{i}$ just before $T, g p_{d}$ is the only reachable parent of $p_{d}$ and $p$ Sibling just before $T$ and $p_{d}$ is the only reachable parent of node $_{d}$ and nodeSibling just before $T$. At $T$, an element of $p_{i}$.child is changed from gp $_{d}=$ node $_{i}$ to newNode.

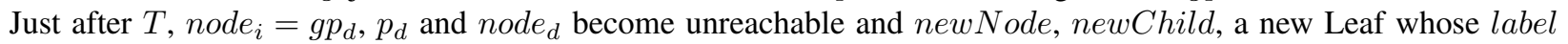
is $v_{a l}$ are reachable. Since nodeSibling and pSibling are children of newNode just after T, nodeSibling and $p$ Sibling are reachable just after $T$. Since node $_{d}$ is the only reachable leaf node containing $v l_{d}$ just before $T$, there is no reachable leaf node containing $v_{a l}$ just after $T$. Thus, $s^{\prime}=s-\left\{v a l_{d}\right\} \cup\left\{v a l_{i}\right\}$.

A replace $\left(v a l_{d}, v a l_{i}\right)$ returns false at line 46 executes a $\operatorname{search}\left(v a l_{d}\right)$ that returns $\left\langle-,-\right.$, node $\left._{d},-,-, r m v d_{d}\right\rangle$ where node $_{d}$ is not a leaf node whose label is $v a l_{d}$, or $r m v d_{d}$ is true. By Lemma 44, there is a time during the $\operatorname{search}\left(v a l_{d}\right)$ when no leaf node whose label is $v_{a l}$ is logically in the trie, so val $\notin$ activeValues at that time. This is the linearization point of $\operatorname{search}\left(v a l_{d}\right)$ and delete $\left(v a l_{d}\right)$ that returns false at line 46 A replace $\left(v a l_{d}\right.$, $\left.v a l_{i}\right)$ returns false at line 48 executes a $\operatorname{search}\left(v a l_{i}\right)$ that returns $\left\langle-,-\right.$, node $\left._{i},-,-, r m v d_{i}\right\rangle$ where node $e_{i}$ is a 
leaf node whose label is $v a l_{i}$ and $\mathrm{rmvd}_{i}$ is false. By the last post-condition of search, there is a time during the $\operatorname{search}\left(v_{a l}\right)$ when node $_{i}$ is logically in the trie, so $v^{2} l_{i} \in$ activeValues at that time. This is the linearization point of $\operatorname{search}\left(v a l_{d}\right)$ and delete $\left(v a l_{d}\right)$ that returns false at line 48 By Corollary 47, if replace $\left(v a l_{d}, v a l_{i}\right)$ returns false, there is no child CAS of $I$ where $I$ is created by replace $\left(v_{a l} l_{d}, v a l_{i}\right)$.

Let $I$ be a Flag object that is created by replace $\left(v a l_{d}, v a l_{i}\right)$. If the first child CAS of $I$ on I.pNode[0] occurs, replace $\left(v a l_{d}, v a l_{i}\right)$ is linearized at that child CAS of $I$. By Lemma 32, the first child CAS of I on I.pNode[0] succeeds and by Lemma 30, no other child CAS of $I$ on I.pNode [0] succeeds. By Lemma 59 and 60, val $\in$ activeValues and $v_{a l} \notin$ activeValues just before the first child CAS of $I$ on I.pNode[0] and val $l_{d}$ is removed from activeValues and $v^{2} l_{i}$ is added to activeValues just after the first child CAS of $I$ on I.pNode[0]. By Lemma 46 if the first child CAS of $I$ on I.pNode[0] occurs, replace $\left(v a l_{d}, v a l_{i}\right)$ returns true (unless it crashes). If $I$ is created at line 55 or 57 and the first child CAS of $I$ on I.pNode[1] occurs, activeValues is not changed at the first child CAS of $I$ on I.pNode[1] (by Lemma 59).

In Section E, we have shown that activeValues is changed correctly just after the linearization points of update operations. By the definition of logically in the trie, activeValues can be changed only by successful child CAS steps. By Lemma 30 and 32, only the first child CAS of $I$ on each element of I.pNode succeeds. We show that activeValues is changed at the first child CAS of $I$ on I.pNode[0] by Lemma 55, 57, 59 and 60 If $I$ is created at line 55 or 57, we show that the first child CAS of I on I.pNode[1] does not change activeValues. So, activeValues is only changed by the child CAS of $I$ on I.pNode[0]. Since activeValues is changed correctly, the find operation returns correct results at its linearization point, according to Lemma 43 and 44 .

\section{F. Progress}

By the lemmas in previous sections, each operation returns the same result as it would if the operations were done in the order of their linearization points. So, operations are linearized correctly. Now, we show the implementation is non-blocking.

First, we show that the search operation is wait-free.

Lemma 61. The search operation is wait-free.

Proof: Let $\ell$ be the length of the keys in $U$. By Invariant 7 , length of node.label increases by at least one in each iteration of loop. Since labels of nodes have length at most $\ell$, there are at most $\ell$ iterations.

Initially, the children of root are two leaf nodes whose labels are $00 \ldots 0$ and $11 \ldots 1$. The following lemma shows that two leaf nodes whose labels are $00 \ldots 0$ and $11 \ldots 1$ are logically in the trie at any time.

Lemma 62. Two leaf nodes whose labels are $00 \ldots 0$ and $11 \ldots 1$ are logically in the trie at all times.

Proof: Initially, root.child is set to two leaf nodes whose labels are $00 \ldots 0$ and $11 \ldots 1$. So, initially, $00 \ldots 0$ and $11 \ldots 1$ are in the activeV alues set. By the definition, only the successful child CAS steps change the activeValues set. By the precondition of update operations, if insert $(\mathrm{val})$, delete $(\mathrm{val})$ is called, $\mathrm{val}$ is not equal to $00 \ldots 0$ or $11 \ldots 1$. By Lemma 48, 55 and 57, no child CAS of $I$ that $I$ is created by an insert operation or a delete operation removes $00 \ldots 0$ or $11 \ldots 1$ from activeValues. By the precondition of replace operation, if replace $\left(v_{a l}, v_{a l}^{\prime}\right)$ is called, val and $v a l^{\prime}$ are not equal to $00 \ldots 0$ or $11 \ldots 1$. By Lemma 48, 59 and 60 no child CAS of $I$ that $I$ is created by a replace operation removes $00 \ldots 0$ or $11 \ldots 1$ from activeValues. So, $00 \ldots 0$ and $11 \ldots 1$ are in the activeValues set at any time. Thus, two leaf nodes whose labels are $00 \ldots 0$ and $11 \ldots 1$ are logically in the trie at all times.

The next lemma shows that all nodes in $F_{I}-U_{I}$ are unreachable after a child CAS step of $I$ on each element of I.pNode succeeds.

Lemma 63. Let I be a Flag object. If the child CAS of I on each element of I.pNode is performed by time T, all nodes in $F_{I}-U_{I}$ are unreachable at all times after $T$.

Proof: Since the first child CAS of $I$ on each element of I.pNode is performed by time $T$, a child CAS of $I$ on each element of I.pNode succeeds by time $T$ (by Lemma 32, If $I$ is created at line 30, 31, 40, 55, 57, 59 or 64. every node in $F_{I}-U_{I}$ is also in I.oldChild. So, if $I$ is not created at line 70, by Lemma 35 and 37 , all nodes in $F_{I}-U_{I}$ are unreachable at all times after $T$.

Now, we show if $I$ is created at line 70, all nodes in $U_{I}-F_{I}$ are unreachable at all times after $T$. Let $\left\langle g p_{d}, p_{d}\right.$, node $\left._{d},-,-,-\right\rangle$ be the result returned by the call to $\operatorname{search}\left(v a l_{d}\right)$ on line 45 that precedes the creation of $I$ and $\langle-$, 
$p_{i}$, node $\left._{i},-,-,-\right\rangle$ be the result returned by the call to $\operatorname{search}\left(v l_{i}\right)$ on line 47 that precedes the creation of $I$. Since $I$ is created at line 70, $F_{I}-U_{I}=\left\{g p_{d}, p_{d}\right\}$. Since I.oldChild $[0]=g p_{d}, g p_{d}$ is unreachable at all times after $T$ (by Lemma 35 and 37). We show that $p_{d}$ becomes unreachable just after the first child CAS of $I$ (which is before $T)$.

Since $p_{i} \in F_{I}, p_{i}$.info $=I$ just before the first child CAS of $I$ (by Corollary 39) and $p_{i}$ is not marked just before the first child CAS of $I$. So, $p_{i}$ is reachable just before the first child CAS of $I$ (by Corollary 34). By Lemma $36 p_{i}$ is a parent of node $e_{i}=g p_{d}$ at some time during $\operatorname{search}\left(v a l_{i}\right)$ and $g p_{d}$ is a parent of $p_{d}$ at some time during $\operatorname{search}\left(v a l_{d}\right)$. So, $p_{i}$ is a parent of $n o d e_{i}=g p_{d}$ and $g p_{d}$ is a parent of $p_{d}$ just before the first child CAS of $I$ (by Lemma 31. Thus, $g p_{d}$ is the only reachable parent of $p_{d}$ just before the first child CAS of $I$ (by Lemma 9 ). Since $g p_{d}$ becomes unreachable just after the first child CAS of $I, p_{d}$ also becomes unreachable just after the first child CAS of $I$. By Lemma 37, $g p_{d}$ is unreachable at all times after $T$.

Lemma 64. Let I be a Flag object. After some call to help(I) terminates, the info field of no reachable internal node is $I$.

Proof: Let $h$ be a call to help $(I)$ that terminates. Now, we show that no flag CAS of $I$ succeeds after $h$ executes line 93 First, $h$ tries to flag at least one node in $F_{I}$ on line 90 (since doChildCAS is initially true). Let $j \geq 1$ be the number of flag CAS steps that $h$ performs. Since $h$ attempts to flag I.flag[1] to I.flag $[j]$ before executing line 93, and only the first flag CAS of $I$ on each node can succeed (by Lemma 16, none of these nodes become flagged after $h$ executes line 93 .

If $x$ is not the last node in I.flag, x.info $\neq I$ on line 91 (since $x$ is the last node in I.flag that $h$ tries to flag). Since the first flag CAS of $I$ on $x$ is performed before $x$.info $\neq I$ on line 91, $x$.info $\neq I$ at all times after that (by Lemma 16. So, after $h$ executes line 93 , no other call to help $(I)$ attempt to flag any node that is in I.flag after $x$. Thus, after $h$ executes line 93, no flag CAS of $I$ succeeds. Now, we consider two cases according to the value of I.flagDone at line 99

Case 1: I.flagDone is false when $h$ executes line 99 Then, $h$ executes a backtrack CAS of $I$ on each element of $F_{I}$ at line 105 Let $x$ be a node in $F_{I}$. If $x$. info $=I$ when $h$ executes line 105, $x$.info is changed to an Unflag object when $h$ executes line 105 and $x$.info is never set back to $I$ after that. If $x$.info $\neq I$ when $h$ executes line 105. $x$.info is not set to $I$ at any time after that (since no flag CAS of $I$ succeeds after that). So, the info field of no node in $F_{I}$ is $I$ after $h$ terminates.

Case 2: I. flagDone is true when $h$ executes line 99 First, we show that a child CAS step of $I$ on each element of I.pNode is performed before $h$ executes line 99. If doChildCAS is true, then $h$ performs these child CAS steps itself. Otherwise, $h$ set $d o C h i l d C A S$ to false at line 91 when the info field of node $x$ is not $I$ on line 91 Since I.flagDone is true when $h$ executes line 99 the info field of $x$ was set to $I$ before $h$ executes line 99 (by Lemma 18). Since $x$.info $\neq I$ when $h$ executes line 91 after $h$ tries to set $x$.info to $I, x$.info was set to $I$ and changed from $I$ to some other value before $h$ executes line 91 (by Lemma 16. So, an unflag or backtrack CAS step of $I$ on $x$ succeeded before $h$ reads $x$.info at line 91 .

Let $h^{\prime}$ be the call to help $(I)$ that executes the first unflag or backtrack CAS of $I$. Since no unflag or backtrack CAS of $I$ succeeds before $h^{\prime}$ executes line 99 and the info fields of all nodes in $F_{I}$ are set to $I$ before $h$ executes line 99. after $h^{\prime}$ tries to flag nodes in $F_{I}$ at line 90, $h^{\prime}$ sets doChildCAS to true at each execution of line 91. Since doChildCAS is true when $h^{\prime}$ executes line 93 . $h^{\prime}$ executes a child CAS of $I$ on each element of I.pNode at line 98 before it performs any unflag or backtrack CAS step of $I$. So, a child CAS of $I$ on each element of I.pNode at line 98 is executed before $h$ reads $x . i n f o$ at line 91 . By Lemma 63. all nodes in $F_{I}-U_{I}$ are unreachable after $h$ terminates.

Since I.flagDone is true when $h$ executes line $99, h$ performs unflag CAS of $I$ on all nodes in $U_{I}$ at line 101 Let $x$ be a node in $U_{I}$. If $x$.info $=I$ when $h$ executes line 101, $x$.info is changed from $I$ to an Unflag object at line 101 and $x$.info is never set back to $I$ after that (by Lemma 15). If $x$.info $\neq I$ when $h$ executes line 101. $x$.info is not set to $I$ at any time after that (since no flag CAS of $I$ succeeds after that). So, the info field of no node in $U_{I}$ is $I$ after $h$ terminates. Thus, the info field of no reachable node is $I$ after $h$ terminates.

The following lemma show that when the update operation reads the node nodeSibling at line 38 or 50 or the node pSibling at line 66, the node was reachable during the update operation at some earlier time. The proof of the lemma is similar to the proof of Lemma 36 and we do not present the argument here again. 
Lemma 65. If the update operation reads nodeSibling at line 38 or 50 during a loop iteration, there is a time after the update operation begins the loop iteration and before the update operation reads nodeSibling that nodeSibling is reachable.

Finally, we show that the implementation is non-blocking.

Lemma 66. The implementation is non-blocking.

Proof: We prove the lemma by contradiction. Assume the implementation is not non-blocking. So, there is an execution of the implementation such that, after time $T$, each pending operation $o p$ take infinitely many steps and no operation terminates.

Assume update operations $o p_{1}, o p_{2}, \ldots, o p_{n}$ each take infinitely many steps after time $T$. By Lemma 61, these operations are update operations.

Each call to help terminates, by Lemma 64, the info field of no reachable node is $I$ after a call to help $(I)$ terminates. So, if $I$ is created before $T$ and $\operatorname{help}(I)$ is called after that, after the call to help $(I)$ terminates, no other operation sees $I$ in the info field of any reachable node. Now, we show that if the operation op calls help $(I)$ during a loop iteration and $I$ is created by another operation, then $I$ was read from the info field of a node that was reachable during the loop iteration.

If $o p$ calls $\operatorname{help}(I)$ and $I$ is created by another operation, then help $(I)$ is called on line 110 or 119 If help $(I)$ is called at line 110, $I$ is read from the info field some node that the search operation returns earlier. If help $(I)$ is called at line 119 inside the createNode routine, which is called at line 27 or 53 , $I$ is read from the info field of some node that the search operation returns earlier. In either case, the node that the search operation returns was reachable at some time during the search operation by Lemma 36 . If help $(I)$ is called at line 119 inside the createNode routine, which is called at line 62, $I$ is read from the info field some node that is read at line 50. By Lemma 65. the node was reachable at some time during the the loop iteration that help $(I)$ is called.

So, if some $o p_{i}$ starts its loop iteration after the first call to help $(I)$ returns, $o p_{i}$ cannot call help $(I)$ during that loop iteration. Since the number of Flag objects that are created before $T$ is finite, there is a time $T_{x}$ after $T$ such that no operation calls help $(I)$ if $I$ is not created by $o p_{1}, o p_{2}, \ldots, o p_{n}$ after $T^{\prime \prime}$. Let $T^{\prime \prime}\left(>T_{x}\right)$ be a time by which each running operation has started a new loop iteration after $T_{x}$. Thus, after $T^{\prime \prime}$, help $(I)$ is only called for Info object $I$ that were created by $o p_{1}, o p_{2}, \ldots, o p_{n}$.

Claim: Some operation calls the help routine after $T^{\prime \prime}$.

Proof of Claim. To derive a contradiction, assume no help routine is called after $T^{\prime \prime}$. So, there is a time $T^{\prime}\left(>T^{\prime \prime}\right)$, no operation executes any line of the help routine. Since the info and child fields of nodes are only changed at line 90, 95, 98, 101 or 105 inside the help routine, the info and child field of no node is changed after $T^{\prime}$. Let $o p$ be an update operation that is running after $T^{\prime}$.

First, we show if $o p$ calls newFlag after $T^{\prime}$, newFlag does not return null. Suppose $o p$ calls newFlag at line 30, 31, 40, 55, 57, 59, 64 or 70. Since no info field is changed after $T^{\prime}$, no newFlag returns null at line 113 If newFlag returns null at line 111 , the help routine is called at line 110 just before that. So, no newFlag returns null after $T^{\prime}$.

We consider different cases according to the type of the update operation that $o p$ is. For each case, we show that op calls the help routine.

Case 1: op is insert $(\mathrm{val})$. We show if $o p$ does not call the help routine at line 32 during a loop iteration after $T^{\prime}$, op calls the help routine at line 119 during that loop iteration. Let $\langle-, p$, node,,,$-- r m v d\rangle$ be the result returned by the call to $\operatorname{search}(v a l)$ at line 23 during the iteration. Since op does not call the help routine at line 32 and newFlag does not return false at line 30 or 31, createNode returns null when it is called at line 27. Suppose createNode node $_{1}$, node $\left._{2},-\right)$ is called at line 27. Then, node ${ }_{1}$ is a new copy of node and node $_{2}$ is a leaf node whose label is val. So, node.label is a prefix of val or val is a prefix of node.label. If node is an internal node, node.label is not a prefix of val by Lemma 6. So, node is a leaf node and node.label =val. Since op does not return false at line 24 rmvd is true. Since rmvd is set to true at line 84 , node.info that is read at line 84 is not an Unflag object. Let $I^{\prime}$ be a Flag object that node.info $=I^{\prime}$ at line 84 By Lemma 40, node.info $=I^{\prime}$ at line 25 Since $I^{\prime}$ is a Flag object, op calls the help routine at line 119

Case 2: $o p$ is delete $(\mathrm{val})$. We show that op calls the help routine at line 41 during each loop iteration after $T^{\prime}$. Let $\langle g p, p$, node, -, -, - $\rangle$ be the result returned by the call to search $(v a l)$ at line 36 during the iteration. First, we 
show $g p$ is not null. If $g p$ is null, the search operation exits the loop after the first loop iteration (since $g p$ is set to $p$ at line 80 during each loop iteration and after the first execution of line 81, $p$ is an internal node by Lemma 4 . Then, $p=$ root. Since op does not return false at line 37, node is a leaf node whose label is val. If node is set to root.child $[0]$ at line 82 , by Lemma 62 and Invariant 7, node.label $=00 \ldots 0$. If node is set to root.child $[1]$ at line 82. by Lemma 62 and Invariant 7 , node.label $=11 \ldots 1$. Since $v a l \neq 00 \ldots 0$ and $v a l \neq 11 \ldots 1$ by the precondition of delete $(v a l), g p$ is not null. Since newFlag does not return null at line 40 op calls the help routine at line 41

Case 3: op is replace $\left(v a l_{d}, v a l_{i}\right)$. Let $\left\langle g p_{d}, p_{d}\right.$, node $\left._{d},-,-,-\right\rangle$ be the result returned by the call to $\operatorname{search}\left(v a l_{d}\right)$ at line 45 during a loop iteration of $o p$ that started after $T^{\prime}$ and $\left\langle-, p_{i}\right.$, node $\left._{i},-,-, r m v d_{i}\right\rangle$ be the result returned by the call to search $\left(v a l_{i}\right)$ at line 47 during the same loop iteration. The same argument as in Case 2 establishes that $g p_{d}$ is not null. Now, we consider different cases of replace $\left(v a l_{d}, v a l_{i}\right)$. For each case, we show that $o p$ calls the help routine.

Case 3A: node $_{i} \notin\left\{\right.$ node $\left._{d}, p_{d}, g p_{d}\right\}$ and $p_{i} \neq p_{d}$. We show if op does not call the help routine at line 71, op calls the help routine at line 119 . Since op does not call the help routine at line 71 and newFlag does not return null at line 55 or 57, neither line 55 nor 57 is executed. So, createNode returns null when it is called at line 53 Suppose createNode $\left(\right.$ node $_{1}$, node $\left._{2},-\right)$ is called at line 53. Then, node $e_{1}$ is a new copy of node $_{i}$ and node $_{2}$ is a leaf node whose label is $v_{a l}$. So, node $e_{i}$.label is a prefix of $v a l_{i}$ or $v a l_{i}$ is a prefix of node $e_{i} . l a b e l$. If node $e_{i}$ is an internal node, node $e_{i}$.label is not a prefix of $v_{a l}$ by Lemma $6 \mathrm{So}$, node $e_{i}$ is a leaf node and node $_{i} . l a b e l=v_{a l}$. Since $o p$ does not return false at line 48, $r m v d_{i}$ is true. Since $r m v d_{i}$ is set to true at line 84, node $e_{i}$.info that is read at line 84 is not an Unflag object. Let $I^{\prime}$ be a Flag object that node .info $=I^{\prime}$ at line 84 By Lemma 40 , node $_{i}$. info $=I^{\prime}$ at line 25 Since $I^{\prime}$ is a Flag object, op calls the help routine at line 119

Case 3B: node $_{i}=$ node $_{d}$. Since newFlag does not return null after $T^{\prime}$, op sets movInfo to a Flag object at line 59 and calls the help routine at line 71 .

Case 3C: node $_{i} \neq$ node $_{d}$ and either node $_{i}=p_{d}$ or $p_{i}=p_{d}$. We show if node $e_{i}=p_{d}, p_{i}=g p_{d}$. By Lemma 6 . $g p_{d}$ was a parent of $p_{d}=$ node $_{i}$ at some time during $\operatorname{search}\left(v a l_{d}\right)$ and $p_{i}$ was a parent of node $e_{i}=g p_{d}$ at some time during $\operatorname{search}\left(v a l_{i}\right)$. Since no child field is changed after $T^{\prime}, g p_{d}$ and $p_{i}$ are parents of $p_{d}=$ node $e_{i}$ at all times after $T^{\prime}$. By Lemma 36, $g p_{d}$ was reachable at some time during search $\left(v a l_{d}\right)$ and $p_{i}$ was reachable at some time during $\operatorname{search}\left(v a l_{i}\right)$. Since no child field is changed after $T^{\prime}, g p_{d}$ and $p_{i}$ are reachable at all times after $T^{\prime}$. So, by Lemma 9 , $p_{i}=g p_{d}$.

Since either node $_{i}=p_{d}$ and $p_{i}=g p_{d}$ or $p_{i}=p_{d}$ (and $g p_{d}$ is not null), createNode is called at line 62 If createNode does not return null, $o p$ calls the help routine at line 71 since newFlag does not return null at line 64 Now, we show if createNode returns null, the help routine is called at line 119 Let nodeSibling be $_{d}$ the child of $p_{d}$ that is read at line 50 Suppose createNode node $_{1}$, node $\left._{2},-\right)$ is called at line 62 Then, node $_{1}$ is nodeSibling $_{d}$

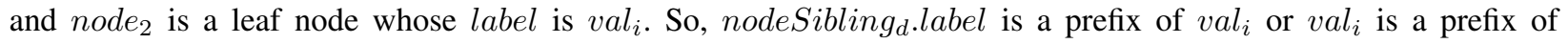
nodeSibling. label. By Lemma 6 , $p_{d}$. child $[i]=$ node $_{d}$ for some $i$ at some time during $\operatorname{search}\left(v l_{d}\right)$.

If node $_{i}=p_{d}$, since node $_{i}$ is an internal node (by Lemma 4), node $e_{i}$ label is not a prefix of $v_{\text {al }}$ (by Lemma

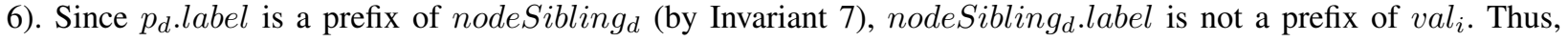
createNode does not return null.

If $p_{i}=p_{d}$, since node $_{i} \neq$ node $_{d}$ and no child field is changed after $T^{\prime}$, node $_{i}$ and node $_{d}$ are children of $p_{i}=p_{d}$ at all times after $T^{\prime}$. Since nodeSibling $g_{d}$ is also a child of $p_{d}$ at all times after $T^{\prime}$ and

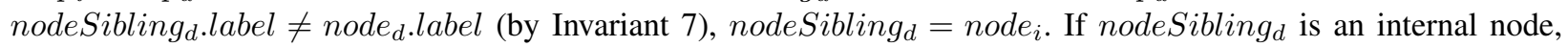
since nodeSibling ${ }_{d}=$ node $_{i}$, nodeSibling. label is not a prefix of val ${ }_{i}$ (by Lemma 6). Then, createNode does not return null. If nodeSibling $g_{d}$ is a leaf node and node $e_{i}$ label $\neq v_{\text {val }}$, createNode does not return null. If nodeSibling $g_{d}$ is a leaf node and node $e_{i} . l a b e l=v a l_{i}$, since $o p$ does not return false at line $48, r m v d_{i}$ is true. Since $r_{m v d}$ is set to true at line 84, node ${ }_{i}$.info is not an Unflag object when it is read at line 84. Let $I^{\prime}$ be a Flag object that node $_{i}$. info $=I^{\prime}$ at line 84 By Lemma 40, since node ${ }_{i}=$ nodeSibling $_{d}$, nodeSibling. info $=I^{\prime}$ at line 62 Since $I^{\prime}$ is a Flag object, op calls the help routine at line 119 .

Case 3D: Otherwise. First, we show that the condition at line 65 is true and node $e_{i}=g p_{d}$. Since the condition at line is not true and, node $_{i}$ is node $e_{d}, p_{d}$ or $g p_{d}$. Since the conditions at line 58 and 60 are not true, then node $_{i} \neq$ node $_{d}$ and node $_{i} \neq p_{d}$. So, node $_{i}=g p_{d}$ and the condition at line 65 is true. Let nodeSibling be the child of $p_{d}$ that is read at line 50 and $p$ Sibling $_{d}$ be the child of $g p_{d}$ that is read at line 66 First, we show that createNode $\left(\right.$ nodeSibling $_{d}$, pSibling $_{d},-$ ) that is called at line 67 does not return null. By Lemma 6 g $g p_{d} . c h i l d[i]=p_{d}$ at some time during $\operatorname{search}\left(v a l_{d}\right)$ for some $i$. By Invariant7. $\left(g p_{d} . l a b e l\right) \cdot i$ is a prefix of $p_{d}$.label. Since nodeSibling 
was a child of $p_{d}$ at line 50. $\left(g p_{d} . l a b e l\right) \cdot i$ is a prefix of nodeSibling.$l a b e l$ (by Invariant 7). By Lemma 6, p p .label is a prefix of $v a l_{d}$. So, $\left(g p_{d} . l a b e l\right) \cdot i$ is a prefix of $v a l_{d}$. Since $p$ Sibling.$l a b e l$ is read as a child of $g p_{d}$ at line 66 .

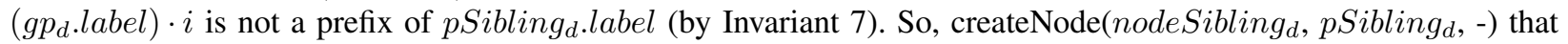
is called at line 67 does not return null.

Since createNode that is called at line 67 does not return null, createNode is also called at line 69 Since $g p_{d}=$ node $_{i}$, node $_{i}$ is an internal node by Lemma 4 and $g p_{d}$.label is not a prefix of $v a l_{i}$ (by Lemma 6. Since $g p_{d} \cdot \operatorname{child}[i]=p_{d}$ at some time during $\operatorname{search}\left(v a l_{d}\right)$ and $p_{d}$ is a parent of nodeSibling $g_{d}$ at line 50, gp $p_{d}$ label is

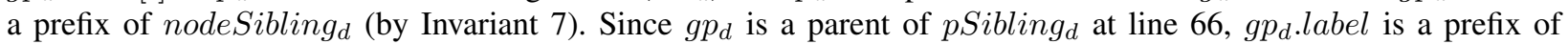
pSibling (by Invariant 7). Let newChild $_{i}$ be the internal node that is created at line 67. Since the children of newChild $_{i}$ are initially nodeSibling and $_{\text {nSibling }}$, gp $_{d}$.label is a prefix of newChild .label (by Invariant 7).

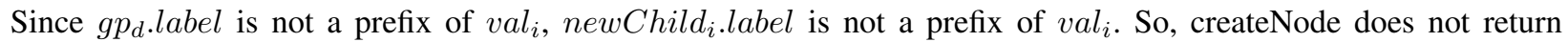
null when it is called at line 69. Since the createNode routines that are called at line 67 and 69 do not return null, $o p$ calls the newFlag routine at line 70. Since newFlag does not return null after $T^{\prime}, o p$ calls the help routine at line 71 This complete the proof of the claim.

Thus, after $T^{\prime}$, some running operation calls the help routine. Let $I$ be a Flag object that is created by some $o p_{i}$ where $1 \leq i \leq n$. After $o p_{i}$ creates $I$, it calls help $(I)$ at line 32, 41 or 71. Any other operation that visits any node whose info field is $I$ might also call help $(I)$ at line 110 or 119 . So, there is a group of calls to help $(I)$ after $T^{\prime}$.

If a child CAS step of $I$ is executed, the operation that created $I$ returns true (by Lemma 46). Since $I$ is created by one of $o p_{1}, o p_{2}, \ldots, o p_{n}$ and $o p_{1}, o p_{2}, \ldots, o p_{n}$ do not return after $T^{\prime}$, no call to help $(I)$ executes a child CAS of $I$ after $T^{\prime}$.

So, no call to help $(I)$ sets I. flagDone to true at line 94 . Thus, all calls to help $(I)$ set doChildCAS to false at line 91. so the info field of some node in $F_{I}$ was not $I$ at line 91 Let node be the first such node that causes any call to help $(I)$ to set doChildCAS to false. We say that a group of calls to help $(I)$ blames node.

Since no child CAS is performed after $T^{\prime}$, the set of nodes logically in the trie does not change. Let node $e_{m}$ be the node in the trie whose label is the greatest among the labels of nodes that are blamed in this way, according to the total ordering that is used to sort on line 115. Let $I_{m}$ be a Flag object such that the group of calls to help $\left(I_{m}\right)$ blames node $_{m}$.

First, we show that node ${ }_{m}$.info is not set to $I_{m}$ by any flag CAS of $I_{m}$. Consider the first time that a call to $\operatorname{help}\left(I_{m}\right)$ sets $d o C h i l d C A S$ to false because node ${ }_{m}$.info $\neq I_{m}$ on line 91 at time $T_{m}$. Since node $_{m}$ is the first node that a call to help $\left(I_{m}\right)$ did not set node $e_{m}$.info to $I_{m}$, node $e_{m}$.info is not set to $I_{m}$ before $T_{m}$. Since the first Flag CAS of $I_{m}$ on node $e_{m}$ occurs before $T_{m}$, no flag CAS of $I_{m}$ changes node ${ }_{m}$.info to $I_{m}$ after $T_{m}$ (by Lemma 16 .

If the old value of node $_{m}$.info that is passed to the newFlag routine is not an Unflag object, the newFlag returns null at line 111. Since $I_{m}$ is created inside the newFlag routine, the old value of node $e_{m}$.info that is passed to the newFlag routine that created $I_{m}$ was an Unflag object. Since no call to help $\left(I_{m}\right)$ flags node $_{m}$ successfully,

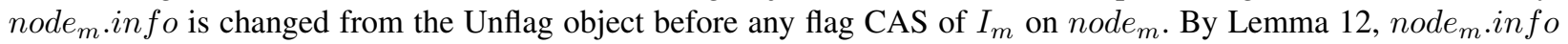
is set to a Flag object $I_{j} \neq I_{m}$ before any flag CAS of $I_{m}$ on node $e_{m}$. Let $g_{j}$ be the group of calls to help $\left(I_{j}\right)$. One such call changes node ${ }_{m}$.info to $I_{j}$. Let node ${ }_{j}$ be the node that $g_{j}$ blames. Then, node .label . node ${ }_{m}$.label by definition of node $_{m}$. (Since the newFlag routine keeps only one copy of duplicates in $I_{j}$. flagNode at line 114. node $_{j}$. label $\neq$ node $_{m}$. label.) However, node $m_{m}$ appears earlier in $I_{j}$. flag array than node $_{j}$, contradicting the fact that nodes in $I_{j}$. flag are flagged in order of their labels and node $e_{j}$.info must set to $I_{j}$ before node ${ }_{m}$.info is set to $I_{j}$ (by Lemma 17).

Therefore, no operation takes infinitely many steps after $T$ and the implementation is non-blocking. 